\title{
Conformal Infinity
}

\author{
Jörg Frauendiener \\ Institut für Theoretische Astrophysik \\ Universität Tübingen \\ Auf der Morgenstelle 10 \\ D-72076 Tübingen \\ Germany \\ email: joergf@tat.physik.uni-tuebingen.de \\ http://www.tat.physik.uni-tuebingen.de/ joergf \\ Accepted on 23 January 2004 \\ Published on 2 February 2004 \\ http://relativity.livingreviews.org/lrr-2004-1 \\ Living Reviews in Relativity \\ Published by the Max Planck Institute for Gravitational Physics \\ Albert Einstein Institute, Germany
}

\begin{abstract}
The notion of conformal infinity has a long history within the research in Einstein's theory of gravity. Today, "conformal infinity" is related to almost all other branches of research in general relativity, from quantisation procedures to abstract mathematical issues to numerical applications. This review article attempts to show how this concept gradually and inevitably evolved from physical issues, namely the need to understand gravitational radiation and isolated systems within the theory of gravitation, and how it lends itself very naturally to the solution of radiation problems in numerical relativity. The fundamental concept of null-infinity is introduced. Friedrich's regular conformal field equations are presented and various initial value problems for them are discussed. Finally, it is shown that the conformal field equations provide a very powerful method within numerical relativity to study global problems such as gravitational wave propagation and detection.
\end{abstract}

(C)2003 Max-Planck-Gesellschaft and the authors.

Further information on copyright is given at http://relativity.livingreviews .org/Info/Copyright/

For permission to reproduce the article please contact livrev@aei.mpg.de. 


\section{Article Amendments}

On author request a Living Reviews article can be amended to include errata and small additions to ensure that the most accurate and up-to-date information possible is provided. For detailed documentation of amendments, please go to the article's online version at

$$
\text { http://www . livingreviews.org//rr-2004-1/ }
$$

Owing to the fact that a Living Reviews article can evolve over time, we recommend to cite the article as follows:

Jörg Frauendiener,

"Conformal Infinity",

Living Rev. Relativity, 7, (2004), 1. [Online Article]: cited [<date>], http://www.livingreviews.org/lrr-2004-1

The date in 'cited $[<$ date $>]$ ' then uniquely identifies the version of the article you are referring to. 


\section{Contents}

1 Introduction $\quad 5$

2 General Background $\quad 7$

2.1 Isolated systems . . . . . . . . . . . . . . . . . . . . . . . . 7

2.2 The emergence of the current picture . . . . . . . . . . . . . . . . . . . . . . . . . . . . . . . .

2.3 Asymptotically flat space-times . . . . . . . . . . . . . . . . . . . . 15

2.4 Example: Minkowski space . . . . . . . . . . . . . . . . . . . . 19

2.5 Going further . . . . . . . . . . . . . . . . . . . . 23

3 The Regular Conformal Field Equations $\quad 25$

3.1 General properties of the conformal field equations . . . . . . . . . . . . . . . . 25

3.2 The reduction process for the conformal field equations . . . . . . . . . . . . . . 28

3.3 Initial value problems . . . . . . . . . . . . . . . . . . . . . . 32

3.4 Hyperboloidal initial data . . . . . . . . . . . . . . . . . . . . . . . 35

3.5 Space-like infinity . . . . . . . . . . . . . . . . . . . . . . . . . 39

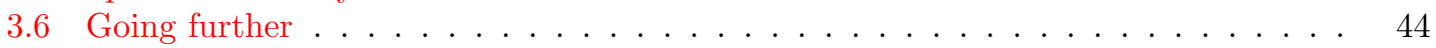

4 Numerical Issues $\quad 45$

4.1 Why use the conformal method for numerical relativity? . . . . . . . . . . . . . 45

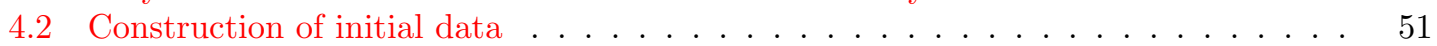

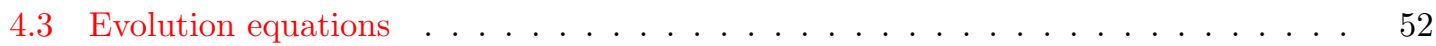

4.4 Some numerical results . . . . . . . . . . . . . . . . . . . . . . 57

4.5 Problems for future considerations . . . . . . . . . . . . . . . . . . 61

5 Acknowledgments $\quad 64$

6 Appendix: Reduction of the Conformal Field Equations $\quad 65$

7 Appendix: Conformal Rescalings and Curvature $\quad \mathbf{7 0}$

$\begin{array}{lr}\text { References } & 71\end{array}$ 



\section{Introduction}

The notion of "conformal infinity" introduced by Penrose almost forty years ago is one of the most fruitful concepts within Einstein's theory of gravitation. Most of the modern developments in the theory are based on or at least influenced in one way or another by the conformal properties of Einstein's equations in general or, in particular, by the structure of null infinity

- the study of radiating solutions of the field equations and the question of fall-off conditions for them;

- the global structure of space-times;

- the structure of singularities;

- conserved quantities;

- multipole moments;

- asymptotic quantization procedures;

- $\mathfrak{H}$-spaces and the twistor programme;

- the null hypersurface formulation of general relativity;

- the conformal field equations and their importance for the numerical evolution of space-times.

Obviously, there exists a vast amount of material related to the subject of conformal infinity that cannot be covered adequately within this review article. A choice has to be made. We will discuss here those issues of "conformal infinity" that are relevant for numerical applications. On the one hand, this is a restriction to a subtopic that is reasonably narrow and yet broad enough to encompass the central ideas and new developments. On the other hand, questions concerning the numerical treatment of gravitational radiation and, in particular, the problems that arise from the attempt to numerically model infinitely extended systems, suggest that the conformal methods can be useful not only for rigorous arguments but also for numerical purposes.

Indeed, we will show that the conformal picture has matured enough to provide an approach to applications in numerical relativity that relies on a very sound theoretical basis not only with respect to the physical appropriateness, but also with respect to the mathematical well-posedness of the problems considered. What is even more remarkable is the fact that the numerical implementations of the conformal picture via the conformal field equations are numerically well-defined in the sense that there are no spurious instabilities in the codes (which so often are the stumbling blocks for the traditional approaches via the ADM equations), so that the computed solutions demonstrably converge to the order of the discretization scheme. The conformal approach based on the hyperboloidal initial-value problem allows us to compute (semi-)global space-times including their asymptotic structure which in turn enables us to rigorously, i.e. without any further approximation beyond the discretization, determine the radiation coming out from the system under consideration. The work that has been devoted to this approach up to now clearly shows its power. The results obtained have been checked against exact results (exact solutions or known theorems), and there is no doubt that the results are correct. The geometric concepts devised by Penrose now turn out to be very useful in practical applications in the sense that they provide the solution to all conceptual problems posed by the notion of "gravitational radiation" in connection with numerical computations. Already, it is obvious that with this tool one can achieve results that have not been feasible by any other numerical method. Furthermore, the analysis at spacelike infinity (see Section 3.5) has finally put within reach the feasibility of computing the global structure of asymptotically flat space-times from asymptotically flat Cauchy data. 
This article will begin with a survey of the general background, i.e. the physical motivations behind the idea of asymptotically flat space-times, and a short account of the historical development that led to our current understanding. Next, the necessary mathematical ideas will be introduced and the regular conformal field equations will be discussed. The equations have been applied to several initial value problems. We will survey the most important results relevant for numerical applications. The last Section 4 is concerned with current issues in the numerical implementation of the conformal methods. We will see that the conformal field equations provide a very powerful method to study global problems in numerical relativity, such as gravitational wave propagation and detection as well as the emergence of singularities and their horizons.

Living Reviews in Relativity

http: //www . livingreviews . org/lrr-2004-1/ 


\section{General Background}

Isolated systems provide valuable insights into the workings of a physical theory by restricting one's attention to small subsystems [79]. They serve as models for systems in the real world, allowing us to deduce statements about their behaviour and to attribute to them various physical properties such as mass, momentum, emitted radiation, etc. Therefore, it is desirable that a theory should allow within its mathematical framework the characterization of such systems. In general relativity, this is a difficult problem. The reason is a familiar one: The metric which, in other theories, provides a background structure on which the physical fields act, is itself a dynamical object in general relativity.

In this section we discuss some of the issues which lead us to focus on asymptotically flat spacetimes as models for isolated systems in relativity and hence as realistic gravitationally radiating systems.

\subsection{Isolated systems}

The conformal structure of space-times has found a wide range of interesting applications in general relativity with various motivations. Of particular importance for us is the emergence of conformal geometric ideas in connection with isolated systems (see also the related discussions in [79, 67]). As an illustration, consider a gravitating system (e.g. a binary system or a star) somewhere in our universe, evolving according to its own gravitational interaction, and possibly reacting to gravitational radiation impinging on it from the outside. Thereby, it will also emit gravitational radiation. We are interested in detecting and evaluating these waves because they provide us with important information about the physics governing the system. For several obvious reasons, it is desirable not only to have a description of such situations within the theoretical framework of the theory but, furthermore, to have the ability to simulate them numerically.

Two problems arise: First, we need to idealize the physical situation in an appropriate way, since it is hopeless to try to analyze the behaviour of the system in its interaction with the rest of the universe. We are mainly interested in the behaviour of the system and not so much in other processes taking place at large distances from the system. Since we would like to ignore those regions, we need a way to isolate the system from their influence.

We might want to do this by cutting away the uninteresting parts of the universe along a time-like cylinder $T$ enclosing the system. Thereby, we effectively replace the outer part by data on $T$. The evolution of our system is determined by those data and initial data on some spacelike hypersurface $S$. But now we are faced with the problem of interpreting the data. It is well known that initial data are obtained from some free data by solving elliptic equations. This is a global procedure. It is very difficult to give a physical meaning to initial data obtained in this way, and it is even more difficult, if not impossible, to specify a system, i.e. to determine initial data, exclusively from (local) physical properties of the constituents of the system like energy-momentum, spin, material properties, and such. In a similar spirit, the data on the timelike boundary $T$ are complicated and only to a rather limited extent do they lend themselves to physical interpretation. For instance, it is not known how to extract from those data any piece that would unambiguously correspond to the radiation emitted by the system. Another problem is related to the arbitrariness in performing the cut. How can we be sure that we capture essentially the same behaviour independently of how we define $T$ ?

Thus, we are led to consider a different kind of "isolation procedure". We imagine the system as being "alone in the universe" in the sense that we assume it being embedded in a space-time manifold that is asymptotically flat. How to formulate this is a priori rather vague. Somehow, we want to express the fact that the space-time "looks like" Minkowski space-time "at large distances" from the source. Certainly, fall-off conditions for the curvature have to be imposed as one recedes 
from the source and these conditions should be compatible with the Einstein equations. This means that there should exist solutions of the Einstein equations that exhibit these fall-off properties. We would then, on some initial space-like hypersurface $S$, prescribe initial data which should, on the one hand, satisfy the asymptotic conditions. On the other hand, the initial data should approximate in an appropriate sense the initial conditions that give rise to the real behaviour of the system. Our hope is that the evolution of these data provides a reasonable approximation of the real behaviour. As before, the asymptotic conditions (which, in a sense, replace the influence of the rest of the universe on the system) should not depend on the particular system under consideration. They should provide some universal structure against which we can gauge the information gained. Otherwise, we would not be able to compare different systems. Furthermore, we would hope that the conditions are such that there is a well defined way to allow for radiation to be easily extracted. It turns out that all these desiderata are in fact realized in the final formulation.

These considerations lead us to focus on space-times that are asymptotically flat in the appropriate sense. However, how should this notion be defined? How can we locate "infinity"? How can we express conditions "at infinity"?

This brings us to the second problem mentioned above. Even if we choose the idealization of our system as an asymptotically flat space-time manifold, we are still facing the task of adequately simulating the situation numerically. This is a formidable task, even when we ignore complications arising from difficult matter equations. The simulation of gravitational waves in an otherwise empty space-time coming in from infinity, interacting with themselves, and going out to infinity is a challenging problem. The reason is obvious: Asymptotically flat space-times necessarily have infinite extent, while computing resources are finite.

The conventional way to overcome this apparent contradiction is the introduction of an artificial boundary "far away from the interesting regions". During the simulation this boundary evolves in time, thus defining a time-like hypersurface in space-time. There one imposes conditions which, it is hoped, approximate the asymptotic conditions. However, introducing the artificial boundary is nothing but the reintroduction of the time-like cylinder $T$ on the numerical level with all its shortcomings. Instead of having a "clean" system that is asymptotically flat and allows well defined asymptotic quantities to be precisely determined, one is now dealing again with data on a time-like boundary whose meaning is unclear. Even if the numerical initial data have been arranged so that the asymptotic conditions are well approximated initially by the boundary conditions on $T$, there is no guarantee that this will remain so when the system is evolved. Furthermore, the numerical treatment of an initial-boundary value problem is much more complicated than an initial value problem because of instabilities that can easily be generated at the boundary.

What is needed, therefore, is a definition of asymptotically flat space-times that allows one to overcome both the problem of "where infinity is" and the problem of simulating an infinite system with finite resources. The key observation in this context is that "infinity" is far away with respect to the space-time metric. This means that one needs infinitely many "metre sticks" in succession in order to "get to infinity". But, what if we replaced these metre sticks by ones that grow in length the farther out we go? Then it might be possible that only a finite number of them suffices to cover an infinite range, provided the growth rate is just right. This somewhat naive picture can be made much more precise: Instead of using the physical space-time metric $\tilde{g}$ to measure distance and time, we use a different metric $g=\Omega^{2} \tilde{g}$, which is "scaled down" with a scale factor $\Omega$. If $\Omega$ can be arranged to approach zero at an appropriate rate, then this might result in "bringing infinity in to a finite region" with respect to the unphysical metric $g$. We can imagine attaching points to the space-time that are finite with respect to $g$ but which are at infinity with respect to $\tilde{g}$. In this way we can construct a boundary consisting of all the end points of the succession of finitely many rescaled metre sticks arranged in all possible directions. This construction works for Minkowski space and so it is reasonable to define asymptotically flat space-times as those for which the scaling-down of the metric is possible.

Living Reviews in Relativity

http://www . livingreviews . org//rr-2004-1/ 
We arrived at this idea by considering the metric structure only "up to arbitrary scaling", i.e. by looking at metrics which differ only by a factor. This is the conformal structure of the space-time manifold in question. By considering the space-time only from the point of view of its conformal structure we obtain a picture of the space-time which is essentially finite but which leaves its causal properties, and hence the properties of wave propagation unchanged. This is exactly what is needed for a rigorous treatment of radiation emitted by the system and also for the numerical simulation of such situations.

The way we have presented the emergence of the conformal structure as the essence of asymptotically flat space-times is not how it happened historically. Since it is rather instructive to see how various approaches finally came together in the conformal picture, we will present in the following Section 2.2 a short overview of the history of the subject.

\subsection{The emergence of the current picture}

The conformal treatment of infinity has grown out of many roots, and it has a history which goes back to the middle of the past century. One of the main motivations was the desire to rigorously understand gravitational waves. The natural question arising was how to characterize wave-like solutions of Einstein's vacuum field equations (allowing for a possible cosmological constant $\lambda$ ):

$$
G_{a b}+\lambda g_{a b}=0
$$

The existence of gravitational waves was predicted by Einstein [42] shortly after he had found the general theory of relativity. However, due to the mathematical and physical complexity of the theory of gravitation there was confusion for a long time about whether the field equations really rigorously do admit solutions with a wave-like character. For instance, Rosen [140] came to the conclusion that there were no such solutions because in the class of plane symmetric waves every non-flat solution of the field equations became singular on a two-dimensional submanifold. This result was, however, due to the lack of understanding of the different kinds of singularities that can occur in a covariant theory, and the singularity appearing in the plane wave space-times later turned out to be a coordinate singularity.

Thus, one of the early problems in the research area of gravitational waves was the invariant characterization of radiation. In 1957, Pirani [136] started the investigation with the suggestion that the algebraic properties of the Riemann (more specifically the Weyl) tensor should be considered as indicating the presence of radiation. In particular, under the assumption that gravitational radiation can in fact be characterized by the curvature tensor and that it propagates with the local speed of light, he proposed the definition that gravitational radiation is present if the curvature tensor has Petrov types $\{211\}$ or $\{31\}$. He arrived at this characterization by the observation that a gravitational wave-front would manifest itself as a discontinuity of the Riemann tensor across null hypersurfaces, these being the characteristics for the Einstein equations. This result had previously been obtained by Lichnerowicz [106]. In his article, Pirani did not enforce the field equations, but towards the end of the paper he proposed to look at the equations $\nabla_{a} R_{b c d}^{a}=0$, which follow from the Bianchi identity for vacuum space-times. This is the first hint at the importance of the Bianchi identity for the study of gravitational waves. In [107] Lichnerowicz proposed a similar definition for a pure gravitational radiation field.

The next important step in the development of the subject was Trautman's study of the question of boundary conditions for the gravitational field equations [157]. He wanted to obtain conditions that were general enough to allow for gravitational radiation from an isolated system of matter, but still strong enough to guarantee uniqueness (appropriately defined) of the solution given "reasonable" initial data. He gave an asymptotic fall-off condition for the metric coefficients with respect to a certain class of coordinate systems. It was obtained by analogy to the situation with the scalar wave equation and Maxwell theory [156], where the fields can be required to satisfy Sommerfeld's 
"Ausstrahlungsbedingung". In those cases it is known that there exist unique solutions for given initial data, while in the gravitational case this was not known at the time and, in fact, remained unknown until only quite recently.

Trautman then went on to discuss some consequences of this boundary condition. He defined an energy-momentum four-vector at infinity which is well defined as a consequence of the boundary condition. It is obtained as the limit of an integral of the energy-momentum pseudo-tensor over a space-like manifold with boundary as it stretches out to infinity. By application of Stokes' theorem, the three-dimensional integral can be converted to a surface integral over the boundary, the sphere at infinity, of certain components of the so-called "superpotentials" for the energymomentum pseudo-tensor. Nowadays, these are recognized as certain special cases of the NesterWitten two-form [44] and, hence, Trautman's energy-momentum integral coincides with the BondiSachs expression or the ADM expression depending on how the limit to infinity is taken. This, however, is not explicitly specified in the paper. He considered the difference of energy-momentum between two space-like hypersurfaces and concluded that it must be due to radiation crossing the time-like cylinder, which together with the two hypersurfaces bounds a four-dimensional volume. An estimate for the amount of radiation showed that it is non-negative. If the limit had been taken out to infinity along null directions, then this result would have coincided with the Bondi-Sachs mass-loss formula.

Finally, Trautman observed that the definitions of pure radiation fields given by Pirani and Lichnerowicz are obeyed not exactly but only asymptotically by gravitational fields satisfying his boundary condition. Hence, he concluded that such solutions approach radiation fields in the limit of large distances to the source.

Based on the ideas of Pirani and Trautman and guided by his own investigations [147] of the structure of retarded linearized gravitational fields of particles, Sachs [142] proposed an invariant condition for outgoing gravitational waves. The intuitive idea was that at large distances from the source, the gravitational field, i.e. the Riemann tensor, of outgoing radiation should have approximately the same algebraic structure as does the Riemann tensor for a plane wave. As one approaches the source, deviations from the plane wave should appear. Sachs analyzed these deviations in detail and obtained rather pleasing qualitative insights into the behaviour of the curvature in the asymptotic regime.

In contrast to the earlier work, Sachs used more advanced geometrical methods. Based on his experience in the study of algebraically special metrics [41, 141, 102] he first analyzed the geometry of congruences of null curves. After the introduction of an appropriate null tetrad he used the Bianchi identity for the curvature tensor in a form that follows from the vacuum field equations to obtain the characteristic fall-off behaviour of the curvature components, which has been termed the "peeling property". The Riemann tensor of a vacuum space-time has this property, if for any given null geodesic with an affine parameter $r$ that extends to infinity, the curvature falls off along the curve in such a way that to order $1 / r$ it is null (Petrov type $\mathrm{N}$ or $\{4\}$ ) with a quadruple PND (principal null direction) along the curve. To order $1 / r^{2}$ it has type III ( $\left.\{31\}\right)$ with the triple PND pointing along the curve, and to order $1 / r^{3}$ it has type II $(\{211\})$ with the double PND oriented along the curve. To order $1 / r^{4}$ it is algebraically general (type I or $\{1111\}$ ) but one of the PND's lies in the direction of the geodesic. To order $1 / r^{5}$ the curvature is not related to the geodesic. Symbolically one can express this behaviour in the form

$$
C=\frac{C_{4}}{r}+\frac{C_{3}}{r^{2}}+\frac{C_{2}}{r^{3}}+\frac{C_{1}}{r^{4}}+\mathcal{O}\left(r^{-5}\right)
$$

where $C$ on the left hand side denotes the Weyl tensor, and each $C_{i}$ on the right hand side stands for a tensor which has an $i$-fold PND along the null geodesic and which is independent of $r$. The important point is that the part of the curvature that has no relation to the null direction of the outgoing geodesic goes as $1 / r^{5}$.

Living Reviews in Relativity

http: //www . livingreviews . org/Irr-2004-1/ 
Sachs postulated the outgoing radiation condition to mean that a bounded source field is free of mixed (i.e. a non-linear superposition of in- and outgoing) radiation at large distances if and only if the field has the peeling property. Later, it was realized $[28,119]$ that this condition does not exclude ingoing radiation. Instead, it is possible to have an ingoing wave profile provided that it falls off sufficiently fast as a function of an advanced time parameter.

The study of gravitational waves and the related questions was the main area of research of the group around Bondi and Pirani at King's College, London, in the years between 1955 and 1967. In a series of papers $[108,109,26,137,110,142,27,144]$ they analyzed several problems related to gravitational waves of increasing complexity. The most important of their results certainly is the work on axi-symmetric radiating systems by Bondi, van der Burg, and Metzner [27]. They used a different approach to the problem of outgoing gravitational waves. Instead of looking at null geodesics they focused on null hypersurfaces, and instead of analyzing the algebraic structure of the curvature using the Bianchi identity they considered the full vacuum field equations. Their work was concerned with axi-symmetric systems, but shortly afterwards Sachs [144] removed this additional assumption.

The essential new ingredient was the use of a retarded time function. This is a scalar function $u$ whose level surfaces are null hypersurfaces opening up towards the future. Based on the assumption that such a function exists, one can introduce an adapted coordinate system, so-called Bondi coordinates, by labeling the generators of the null hypersurfaces with coordinates on the two-sphere and introducing the luminosity distance $r$ (essentially the square root of the area of outgoing wave fronts) along the null generators. The metric, when written in this kind of coordinate system, contains only six free functions.

Asymptotic conditions were imposed to the effect that one should be able to follow the null geodesics outwards into the future for arbitrarily large values of $r$. Then the metric was required to approach the flat metric in the limit of infinite distance. Additionally, it was assumed that the metric functions and other quantities of interest (in particular the curvature) were analytic functions of $1 / r$.

The field equations in Bondi coordinates have a rather nice hierarchical structure that is symptomatic for the use of null coordinates and that allows for a simpler formal analysis compared to the related Cauchy problem. Bondi et al. and Sachs were able to solve the field equations asymptotically for large distances. In essence their procedure amounts to the formulation of a certain characteristic initial value problem (see $[145,146]$ ) and the identification of the free data. It turns out that the freely specifiable data are two functions, essentially components of the metric, on an initial null hypersurface $u=u_{0}$ and two similar functions at " $r=\infty$ ". These latter functions are Bondi's news functions whose non-vanishing is taken to indicate the presence of gravitational radiation.

The results of this analysis were very satisfactory and physically reasonable. The most important consequence is the demonstration that outgoing gravitational waves carry away energy from the source and hence diminish its mass. This is the consequence of the Bondi-Sachs mass loss formula, which relates the rate of the mass decrease to the integral over the absolute value of the news. Another consequence of the analysis was the peeling property: For space-times which satisfy the vacuum field equations and the Bondi-Sachs boundary conditions, the curvature necessarily has the asymptotic behaviour (1) as predicted by Sachs' direct analysis of the Riemann tensor using the vacuum Bianchi identity. Thus, the Bondi-Sachs conditions imply the covariant outgoing radiation condition of Sachs and also the boundary condition proposed by Trautman.

The group of coordinate transformations that preserve the form of the metric and the boundary conditions was determined. This infinite-dimensional group, which became known as the BMS group, is isomorphic to the semi-direct product of the homogeneous Lorentz group with the Abelian group of so-called super-translations. The emergence of this group came as a surprise because one would have expected the Poincaré group as the asymptotic symmetry group, but one obtained a 
strictly larger group. However, the structure of the BMS group is quite similar to the Poincaré group. In particular, it contains a unique Abelian normal subgroup of four dimensions, which can be identified with the translation group. This result forms the basis of further investigations into the nature of energy-momentum in general relativity. The BMS group makes no reference to the metric that was used to derive it. Therefore, it can be interpreted as the invariance group of some universal structure that comes with every space-time satisfying the Bondi-Sachs boundary conditions. The BMS group has been the subject of numerous further investigations since then. For some of them we refer to [112, 113, 114, 118, 128, 135, 143].

At about the same time, Newman and Penrose [117] had formulated what has become known as the NP formalism. It combined the spinor methods, which had been developed earlier by Penrose [124], with the (null-)tetrad calculus used hitherto. Newman and Penrose applied their formalism to the problem of gravitational radiation. In particular, they constructed a coordinate system that was very similar to the ones used by Bondi et al. and Sachs. The only difference was their use of an affine parameter instead of luminosity distance along the generators of the null hypersurfaces of constant retarded time. Based on these coordinates and an adapted null frame, they showed that the single assumption $\Psi_{0}=\mathcal{O}\left(1 / r^{5}\right.$ ) (and the technical assumption of the uniformity of the angular derivatives) already implied the peeling property as stated by Sachs. The use of $\Psi_{0}$ as the quantity whose properties are specified on a null hypersurface was in accordance with a general study of characteristic initial value problems for spinor equations and in particular for general relativity undertaken by Penrose [130].

An important point in this work was the realization that the Bianchi identity could be regarded as a field equation for the Weyl tensor. It might be useful here to point out that it is a misconception to consider the Bianchi identity as simply a tautology and to ignore it as contributing no further information, as is often done even today. It is an important piece of the structure on a Riemannian or Lorentzian manifold, relating the (derivatives of the) Ricci and Weyl tensors. If the Ricci tensor is restricted by the Einstein equations to equal the energy-momentum tensor, then the Bianchi identity provides a differential equation for the Weyl tensor. Its structure is very similar to the familiar zero rest-mass equation for a particle with spin 2. In fact, in a sense one can consider this equation as the essence of the gravitational theory.

Newman and Unti [121] carried the calculations started in [117] further and managed to solve the full vacuum field equations asymptotically for large distances. The condition of asymptotic flatness was imposed not on the metric but directly on the Weyl tensor in the form suggested by Newman and Penrose, namely that the component $\Psi_{0}$ of the Weyl tensor should have the asymptotic behaviour $\Psi_{0}=\Psi_{0}^{0} / r^{5}+\mathcal{O}\left(1 / r^{6}\right)$. From this assumption alone (and some technical requirements similar to the ones mentioned above) they obtained the correct peeling behaviour of the curvature, the form of the metric up to the order of $1 / r^{5}$, in particular its flatness at large distances, and also the Bondi-Sachs mass loss formula. Later, the procedure developed by Newman and Unti to integrate the vacuum field equations asymptotically was analyzed by Dixon [40], who showed that it could be carried out consistently to all orders in $1 / r$.

It is remarkable how much progress could be made within such a short time (only about four years). The trigger seems to have been the use of the structure of the light cones in one form or another in order to directly describe the properties of the radiation field: the introduction of the retarded time function, the use of an adapted null-tetrad, and the idea to "follow the field along null directions". This put the emphasis onto the conformal structure of space-times.

The importance of the conformal structure became more and more obvious. Schücking had emphasized the conformal invariance of the massless free fields, a fact that had been established much earlier by Bateman [16] and Cunningham [38] for the wave equation and the free Maxwell field, and by McLennan [115] for general spin. This had led to the idea that conformal invariance might play a role also in general relativity and, in particular, in the asymptotic behaviour of the gravitational radiation field (see [133] for a personal account of the development of these ideas).

Living Reviews in Relativity

http://www. livingreviews . org//rr-2004-1/ 
Finally, Penrose [125] outlined a completely different point of view on the subject, arrived at by taking the conformal structure of space-time as fundamental. He showed that if one regarded the metric of Minkowski space-time to be specified only up to conformal rescalings $g \mapsto \Omega^{2} g$ for some arbitrary function $\Omega$, then one could treat points at infinity on the same basis as finite points. Minkowski space-time could be completed to a highly symmetrical conformal manifold by adding a "null-cone at infinity". The well known zero rest-mass fields which transform covariantly under conformal rescalings of the metric are well defined on this space, and the condition that they be finite on the null-cone at infinity translates into reasonable fall-off conditions for the fields on Minkowski space. On the infinite null-cone one could prescribe characteristic data for the fields that correspond to the strength of their radiation field. He suggested that asymptotically flat space-times should share at least some of these properties.

This point of view proved successful. In a further paper by Penrose [126], the basic qualitative picture we have today is developed. Roughly speaking (see the next Section 3 for a detailed account), the general idea is to attach boundary points to the "physical" space-time manifold that idealize the end-points at infinity reached by infinitely extended null geodesics. This produces a manifold with boundary, the "unphysical" manifold, whose interior is diffeomorphic to the physical manifold. Its boundary is a regular hypersurface whose causal character depends on the cosmological constant. The unphysical manifold is equipped with a metric that is conformal to the physical metric with a conformal factor $\Omega$ which vanishes on the boundary. In addition, the structure of the conformal boundary is uniquely determined by the physical space-time.

Let us illustrate this with a simple example. The metric of Minkowski space-time in polar coordinates is

$$
\tilde{g}=d t^{2}-d r^{2}-r^{2} d \sigma^{2},
$$

where $d \sigma^{2}$ is the metric of the unit sphere. To perform the conformal rescaling we introduce null coordinates $u=t-r$ and $v=t+r$. This puts the Minkowski line-element into the form

$$
\tilde{g}=d u d v-\frac{1}{4}(v-u)^{2} d \sigma^{2},
$$

where $d \sigma^{2}$ is the metric of the unit sphere. The coordinates $u$ and $v$ each range over the complete real line, subject only to the condition $v-u \geq 0$. This infinite range is compactified by transforming with an appropriate function, e.g.

$$
u=\tan U, \quad v=\tan V,
$$

thus introducing new null coordinates $U$ and $V$, in terms of which the metric takes the form

$$
\tilde{g}=\frac{1}{4 \cos ^{2} U \cos ^{2} V}\left[4 d U d V-\sin ^{2}(V-U) d \sigma^{2}\right] .
$$

The coordinates $U, V$ both range over the open interval $(-\pi / 2, \pi / 2)$ with the restriction $V-U \geq 0$. Obviously, the Minkowski metric is not defined at points with $U= \pm \pi / 2$ or $V= \pm \pi / 2$. Any extension of the metric in this form will be singular.

Now we define a different metric $g=\Omega^{2} \tilde{g}$, conformally related to $\tilde{g}$ by the conformal factor $\Omega=2 \cos U \cos V$. Thus,

$$
g=4 d U d V-\sin ^{2}(V-U) d \sigma^{2} .
$$

This metric is perfectly regular at the points mentioned above and, in fact, $g$ is the metric of the Einstein cylinder $\mathcal{E}$. This can be verified by defining an appropriate time and radius coordinate. With $T=U+V, R=V-U$ we have

$$
g=d T^{2}-d R^{2}-\sin ^{2} R d \sigma^{2} .
$$




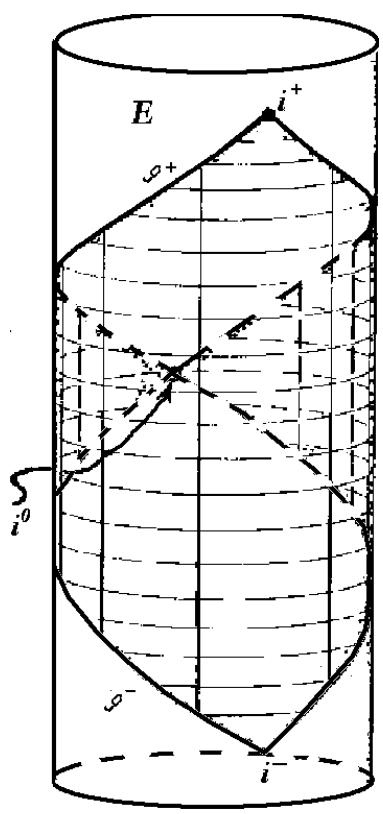

Figure 1: The embedding of Minkowski space into the Einstein cylinder $\mathcal{E}$.

Thus, we may consider Minkowski space to be conformally embedded into the Einstein cylinder. This is shown in Figure 1. The Minkowski metric determines the structure of the boundary, namely the two three-dimensional null-hypersurfaces $\mathscr{I}^{+}$and $\mathscr{I}^{-}$which represent (future and past) "nullinfinity". This is where null-geodesics "arrive". They are given by the conditions $V=\pi / 2,|U|<$ $\pi / 2\left(\mathscr{I}^{+}\right)$and $U=-\pi / 2,|V|<\pi / 2\left(\mathscr{I}^{-}\right)$. The points $i^{ \pm}$are given by $U=V= \pm \pi / 2$. They represent "future and past time-like infinity", the start and the end-point, respectively, of time-like geodesics, while $i^{0}$ is a point with $U=-\pi / 2, V=\pi / 2$. It is the start and end-point of all space-like geodesics, hence, it represents "space-like infinity".

The conformal boundary of Minkowski space-time consists of the pieces $\mathscr{I}^{ \pm}, i^{ \pm}$, and $i^{0}$. These are fixed by the Minkowski metric. In contrast to this, the conformal manifold into which Minkowski space-time is embedded (here the Einstein cylinder) is not fixed by the metric. Obviously, had we chosen a different conformal factor $\hat{\Omega}=\theta \Omega$ with some arbitrary positive function $\theta$ we would not have obtained the metric of the Einstein cylinder but a different one. We see from this that although the conformal boundary is unique, the conformal extension beyond the boundary is not.

The conformal compactification process is useful for several reasons. First of all, it simplifies the discussion of problems at infinity that would involve complicated limit procedures when viewed with respect to the physical metric. Transforming to the unphysical metric attaches a boundary to the manifold so that issues which arise at infinity with respect to the physical metric can be analyzed by local differential geometric arguments in the neighbourhood of the boundary.

This is particularly useful for discussing solutions of conformally invariant field equations on space-time. The basic idea is the following: Consider a space-time that allows us to attach a conformal boundary, thus defining an unphysical manifold conformally related to the given spacetime. Suppose we are also given a solution of a conformally invariant equation on this unphysical manifold. Because of the conformal invariance of the equation, there exists a rescaling of that unphysical field with a power of the conformal factor, which produces a solution of the equation on the physical manifold. Now, suppose that the unphysical field is smooth on the boundary. Then

Living Reviews in Relativity

http: //www. livingreviews.org/lrr-2004-1/ 
the physical solution will have a characteristic asymptotic behaviour that is entirely governed by the conformal weight of the field, i.e. by the power of the conformal factor used for the rescaling. Thus, the regularity requirement of the unphysical field translates into a characteristic asymptotic fall-off or growth behaviour of the physical field, depending on its conformal weight.

Penrose used this idea to show that solutions of the zero rest-mass equations for arbitrary spin on a space-time, which can be compactified by a conformal rescaling, exhibit the peeling property in close analogy to the gravitational case as discovered by Sachs. Take as an example the spin-2 zero rest-mass equation for a tensor $K_{a b c d}$ with the algebraic properties of the Weyl tensor,

$$
\nabla^{a} K_{a b c d}=0 .
$$

This is the equation for linear perturbations of the gravitational fields propagating on a fixed background. It is conformally covariant, in the sense that it remains unchanged provided the field is rescaled as $K_{a b c d} \mapsto \Omega^{-1} K_{a b c d}$, i.e. it is a conformal density with weight -1 .

Using the geometric technique of conformal compactification, Penrose was able to establish the peeling property also for general (non-linear) gravitational fields. We will discuss this result explicitly in the following Section 2.3. Furthermore, he showed that the group of transformations of the conformal boundary leaving the essential structure invariant was exactly the BMS group. This geometric point of view suggested that the asymptotic behaviour of the gravitational field of an isolated radiating gravitational system can be described entirely in terms of its conformal structure. The support for this suggestion was overwhelming from an aesthetical point of view, but a rigorous support for this claim was provided essentially only from the examination of the formal expansion type solutions of Bondi-Sachs and Newman-Unti and the analysis of explicit stationary solutions of the field equations.

The geometric point of view outlined above is the foundation on which many modern developments within general relativity are based. Let us now discuss the notion of asymptotically flat space-times and some of their properties in more detail.

\subsection{Asymptotically flat space-times}

We have seen in Section 2.1 that the question of how to define isolated systems in general relativity has led to the mathematical idealization of asymptotically flat space-times. They are defined by the requirement that they allow the attachment of a smooth conformal boundary. The precise definition is:

Definition 1: A smooth (time- and space-orientable) space-time $\left(\widetilde{\mathcal{M}}, \tilde{g}_{a b}\right)$ is called asymptotically simple, if there exists another smooth Lorentz manifold $\left(\mathcal{M}, g_{a b}\right)$ such that

(1) $\widetilde{\mathcal{M}}$ is an open submanifold of $\mathcal{M}$ with smooth boundary $\partial \widetilde{\mathcal{M}}=\mathscr{I}$;

(2) there exists a smooth scalar field $\Omega$ on $\mathcal{M}$, such that $g_{a b}=\Omega^{2} \tilde{g}_{a b}$ on $\widetilde{\mathcal{M}}$, and so that $\Omega=0$, $d \Omega \neq 0$ on $\mathscr{I}$

(3) every null geodesic in $\widetilde{\mathcal{M}}$ acquires a future and a past endpoint on $\mathscr{I}$.

An asymptotically simple space-time is called asymptotically flat, if in addition $\tilde{R}_{a b}=0$ in a neighbourhood of $\mathscr{I}$.

Thus, asymptotically flat space-times are a subclass of asymptotically simple space-times, namely those for which the Einstein vacuum equations hold near $\mathscr{I}$. Examples of asymptotically simple space-times that are not asymptotically flat include the de Sitter and anti-de Sitter space-times, both solutions of the Einstein equations with non-vanishing cosmological constant. We will concentrate here on asymptotically flat space-times. 
According to Condition (1) in Definition 1, the space-time $\left(\widetilde{\mathcal{M}}, \tilde{g}_{a b}\right)$, which we call the physical space-time, can be considered as part of a larger space-time $\left(\mathcal{M}, g_{a b}\right)$, the unphysical space-time. As a submanifold of $\mathcal{M}$, the physical space-time can be given a boundary which is required to be smooth. The unphysical metric $g_{a b}$ is well-defined on $\mathcal{M}$ and, in particular, on $\widetilde{\mathcal{M}}$, while the physical metric $\tilde{g}_{a b}$ is only defined on $\widetilde{\mathcal{M}}$ and cannot be extended in a well-defined sense to the boundary of $\widetilde{\mathcal{M}}$ or even beyond. The metrics generate the same conformal structure; they are conformally equivalent in the sense that on $\widetilde{\mathcal{M}}$ they define the same null-cone structure.

Note that although the extended manifold $\mathcal{M}$ and its metric are called unphysical, there is nothing unphysical about this construction. As we shall see below, the boundary of $\widetilde{\mathcal{M}}$ in $\mathcal{M}$ is uniquely determined by the conformal structure of $\widetilde{\mathcal{M}}$ and, therefore, it is just as physical as $\widetilde{\mathcal{M}}$. The extension beyond the boundary, given by $\mathcal{M}$, is not unique, as we have already seen in Section 2.2 , but this is of no consequence for the physics in $\widetilde{\mathcal{M}}$ because the extension is causally disconnected from $\widetilde{\mathcal{M}}$.

Condition (2) in Definition 1 fixes the behaviour of the scaling factor on $\mathscr{I}$ as being "of the order $1 / r$ " as one approaches $\mathscr{I}$ from within $\widetilde{\mathcal{M}}$. Condition (3) in Definition 1 is a completeness condition to ensure that the entire boundary is included. In some cases of interest, this condition is not satisfied. In the Schwarzschild space-time, for instance, there are null-geodesics that circle around the singularity, unable to escape to infinity. This problem has led to a weakening of Definition 1 to weakly asymptotically simple space-times (see e.g. [127]). Such space-times are essentially required to be isometric to an asymptotically simple space-time in a neighbourhood of the boundary $\mathscr{I}$. A different completeness condition has been proposed by Geroch and Horowitz [81]. In the following discussion of the analytic and geometric issues, weakly asymptotically simple space-times will not play a role so that we can assume our space-times to be asymptotically simple. Of course, for applications weakly asymptotically simple space-times are important because they provide interesting examples of space-times with black holes.

We defined asymptotically flat space-times by the requirement that the Einstein vacuum equation holds near the boundary, i.e. that asymptotically the physical space-time is empty. There are ways to relax this condition by imposing strong enough fall-off conditions on the energy-momentum tensor without violating any of the consequences. For example, it is then possible to include electromagnetic fields. Since we are concerned here mainly with the asymptotic region, we are not really interested in including any matter fields. Therefore, we will assume henceforth that the physical space-time is a vacuum space-time. This does not mean that the following discussion is only valid for vacuum space-times; it simply allows us to make simpler statements.

The conformal factor $\Omega$ used to construct the boundary $\mathscr{I}$ is, to a large extent, arbitrary. It is fixed only by its properties on the boundary. This raises the important question about the uniqueness of the conformal boundary as a point set and as a differential manifold. If this uniqueness were not present, then the notion of "points at infinity" would be useless. It could then happen that two curves that approach the same point in one conformal boundary for a space-time reach two different points in another conformal completion. Or, similarly, that two conformal extensions that arise from two different conformal factors were not smoothly related. However, these problems do not arise. In fact, it can be shown that between two smooth extensions there always exists a diffeomorphism which is the identity on the physical space-time, so that the two extensions are indistinguishable from the point of view of their topological and differential structure. This was first proved by Geroch [75]. It also follows from Schmidt's so-called b-boundary construction $[148,149,151]$.

From the condition that the vacuum Einstein equation holds, one can derive several important consequences for asymptotically flat space-times:

Living Reviews in Relativity

http://www. livingreviews .org/lrr-2004-1/ 
(1) $\mathscr{I}$ is a smooth null hypersurface in $\mathcal{M}$.

(2) $\mathscr{I}$ is shear-free.

(3) $\mathscr{I}$ has two connected components, each with topology $S^{2} \times \mathbb{R}$.

(4) The conformal Weyl tensor vanishes on $\mathscr{I}$.

The first part of Statement (1) follows from the fact that $\mathscr{I}$ is given by the equation $\Omega=0$. Since $\Omega$ has a non-vanishing gradient on $\mathscr{I}$, regularity follows. Furthermore, from the Einstein vacuum equations one has $\tilde{\Lambda}=0$ on $\widetilde{\mathcal{M}}$. Hence, Equation (114) implies on $\widetilde{\mathcal{M}}$ :

$$
\Omega^{2} \Lambda-\frac{1}{4} \Omega \square \Omega+\frac{1}{2} \nabla^{a} \Omega \nabla_{a} \Omega=0 .
$$

This equation can be extended smoothly to the boundary of $\widetilde{\mathcal{M}}$, yielding there the condition $N_{a} N^{a}=0$ for the co-normal $N_{a}=-\nabla_{a} \Omega$ of $\mathscr{I}$. Hence, the gradient of the conformal factor is null, and $\mathscr{I}$ is a null hypersurface.

As such it is generated by null geodesics. The Statement (2) asserts that the congruence formed by the generators of $\mathscr{I}$ has vanishing shear. To show this we look at Equation (113) and find from $\tilde{\Phi}_{a b}=0$ that

$$
\Omega \Phi_{a b}+\nabla_{a} \nabla_{b} \Omega-\frac{1}{4} g_{a b} \square \Omega=0
$$

whence, on $\mathscr{I}$ we get (writing $m_{a b}$ for the degenerate induced metric on $\mathscr{I}$ )

$$
2 \nabla_{a} N_{b}=\mathcal{L}_{N} m_{a b}=-\frac{1}{2} m_{a b} \square \Omega,
$$

whence the Lie-derivative of $m_{a b}$ along the generators is proportional to $m_{a b}$, which is the shear-free condition for null geodesic congruences with tangent vector $N^{a}$ (see $[88,135]$ ).

To prove Statement (3) we observe that since $\mathscr{I}$ is null, either the future or the past light cone of each of its points has a non-vanishing intersection with $\widetilde{\mathcal{M}}$. This shows that there are two components of $\mathscr{I}$, namely $\mathscr{I}^{+}$on which null geodesics attain a future endpoint, and $\mathscr{I}^{-}$where they attain a past endpoint. These are the only connected components because there is a continuous map from the bundle of null-directions over $\widetilde{\mathcal{M}}$ to $\mathscr{I}^{ \pm}$, assigning to each null direction at each point $P$ of $\widetilde{\mathcal{M}}$ the future (past) endpoint of the light ray emanating from $P$ in the given direction. If $\mathscr{I}^{ \pm}$were not connected then neither would be the bundle of null-directions of $\widetilde{\mathcal{M}}$, which is a contradiction $\left(\widetilde{\mathcal{M}}\right.$ being connected). To show that the topology of $\mathscr{I}^{ \pm}$is $S^{2} \times \mathbb{R}$ requires a more sophisticated argument, which has been given by Penrose [126] (a different proof has been provided by Geroch [78]). It has been pointed out by Newman [122] that these arguments are only partially correct. He rigorously analyzed the global structure of asymptotically simple space-times and he found that, in fact, there are more general topologies allowed for $\mathscr{I}$. However, his analysis was based on methods of differential topology not taking the field equations into account. Indeed, we will find later in Theorem 6 that the space-time that evolves from data close enough to Minkowski data will have a $\mathscr{I}^{+}$with topology $S^{2} \times \mathbb{R}$.

The proof of Statement (4) depends in an essential way on the topological structure of $\mathscr{I}$. We refer again to [126]. The vanishing of the Weyl curvature on $\mathscr{I}$ is the final justification for the definition of asymptotically flat space-times: Vanishing Ricci curvature implies the vanishing of the Weyl tensor and hence of the entire Riemann tensor on $\mathscr{I}$. The physical space-time becomes flat at infinity.

But there is another important property that follows from the vanishing of the Weyl tensor on $\mathscr{I}$. Consider the Weyl tensor $C^{a} b c d$ of the unphysical metric $g_{a b}$, which agrees on $\widetilde{\mathcal{M}}$ with the Weyl tensor $\tilde{C}_{b c d}^{a}$ of the physical metric $\tilde{g}_{a b}$ because of the conformal invariance (110). On $\widetilde{\mathcal{M}}$, $\tilde{C}^{a} b c d$ satisfies the vacuum Bianchi identity,

$$
\tilde{\nabla}_{a} \tilde{C}_{b c d}^{a}=0 .
$$


This equation looks superficially like the zero rest-mass equation (8) for spin-2 fields. However, the conformal transformation property of Equation (10) is different from the zero rest-mass case. The equation is not conformally invariant since the conformal rescaling of a vacuum metric generates Ricci curvature in the unphysical space-time by Equation (111), which then feeds back into the Weyl curvature via the Bianchi identity (cf. Equation (115)). However, we can define the field

$$
K_{b c d}^{a}=\Omega^{-1} C_{b c d}^{a}
$$

on $\widetilde{\mathcal{M}}$. As it stands, $K_{b c d}^{a}$ is not defined on $\mathscr{I}$. But the vanishing of the Weyl tensor there and the smoothness assumption allow the extension of $K_{b c d}^{a}$ to the boundary (and even beyond) as a smooth field on $\mathcal{M}$. It follows from Equation (10) that this field satisfies the zero rest-mass equation

$$
\nabla_{a} K_{b c d}^{a}=0
$$

on the unphysical space-time $\mathcal{M}$ with respect to the unphysical metric. Therefore, the rescaled Weyl tensor $K^{a}{ }_{b c d}$ is a genuine spin-2 field with the natural conformal behaviour. In fact, this is the field that most directly describes the gravitational effects; in particular, its values on the boundary are closely related to the gravitational radiation that escapes from the system under consideration. It propagates on the conformal space-time in a conformally covariant way according to Equation (11) which looks superficially like the equation (8) for a (linear) spin-2 zero rest-mass field. However, there are highly non-linear couplings between the connection given by $\nabla_{a}$ and the curvature given by $K_{a b c d}$. In the physical space-time, where the conformal factor is unity, the field $K_{a b c d}$ coincides with the Weyl tensor, which is the source of tidal forces acting on test particles moving in space-time. For these reasons, we will call the rescaled Weyl tensor $K_{a b c d}$ the gravitational field.

From Equation (11) and the regularity on $\mathscr{I}$ follows a specific fall-off behaviour of the field $K^{a}{ }_{b c d}$, and hence of the Weyl tensor, which is exactly the peeling property obtained by Sachs. It arises here from a reasoning similar to the one presented towards the end of Section 2.2. It is a direct consequence of the geometric assumption that the conformal completion be possible and of the conformal invariance of Equation (11). This equation for the rescaled Weyl tensor is an important sub-structure of the Einstein equation because it is conformally invariant, in contrast to the Einstein equation itself. In a sense it is the most important part also in the system of conformal field equations, which we consider in the next Section 3.

The possibility of conformal compactification restricts the lowest order structure of the gravitational field on the boundary. This means that all asymptotically flat manifolds are the same in that order, so that the conformal boundary and its structure are universal features among asymptotically flat space-times. The invariance group of this universal structure is exactly the BMS group. Differences between asymptotically flat space-times can arise only in a higher order. This is nicely illustrated by the Weyl tensor, which necessarily vanishes on the conformal boundary, but the values of the rescaled Weyl tensor $K_{a b c d}=\Omega^{-1} C_{a b c d}$ are not fixed there.

In summary, our qualitative picture of asymptotically flat space-times is as follows: Such spacetimes are characterized by the property that they can be conformally compactified. This means that we can attach boundary points to all null-geodesics. More importantly, these points together form a three-dimensional manifold that is smoothly embedded into a larger extended space-time. The physical metric and the metric on the compactified space are conformally related. Smoothness of the resulting manifold with boundary translates into asymptotic fall-off conditions for the physical metric and the fields derived from it. The boundary emerges here as a geometric concept and not as an artificial construct put in by hand. This is reflected by the fact that it is not possible to impose a "boundary condition" for solutions of the Einstein equations there. In this sense it was (and is) not correct to talk about a "boundary condition at infinity" as we and the early works sometimes did.

Living Reviews in Relativity

http://www. livingreviews.org//rr-2004-1/ 


\subsection{Example: Minkowski space}

In this section we continue our discussion of the prototype of an asymptotically flat space-time, namely Minkowski space-time. The motivation for doing so is partly to get more acquainted with the idea of conformal compactification and partly to show why this concept is the correct one for the description of radiation processes.

For the following discussion we refer to the rescaled metric

$$
g=4 d U d V-\sin ^{2}(V-U) d \sigma^{2},
$$

which is the metric of the Einstein cylinder. The standard conformal diagram for Minkowski space [126] is shown in Figure 2.



Figure 2: The conformal diagram of Minkowski space.

Each point in the interior of the triangle corresponds to a 2-sphere. The long side of the triangle consists of all the points in the centre, $r=0$ (i.e. $U=V$ ). The other two sides of the triangle correspond to null-infinity $\mathscr{I}^{ \pm}$. The points $i^{ \pm}$are points in the centre with $U=V= \pm \pi / 2$, while $i^{0}$ is a point with $U=-\pi / 2, V=\pi / 2$. The lines meeting at $i^{0}$ are lines of constant $t$, while the lines emanating from $i^{-}$and converging into $i^{+}$are lines of constant $r$. In the four-dimensional space-time the lines of constant $t$ correspond to three-dimensional space-like hypersurfaces that are asymptotically Euclidean.

In the case of Minkowski space-time, the metric can be extended in a regular way to three points representing future and past time-like infinity and space-like infinity, but this is not generally so. Already in the case of the Schwarzschild metric, which is also an asymptotically flat metric, there are, strictly speaking, no such points because any attempt to extend the metric yields a singularity. However, it is common language to refer to this behaviour by saying that "the points $i^{0}$ and/or $i^{ \pm}$ are singular". The reason for this is related to the presence of mass. For any space-time that has a 
non-vanishing ADM mass, the point $i^{0}$ is necessarily singular while the singularity of the time-like infinities is, in general, related to the fall-off properties of the energy-momentum tensor in time-like directions. In the case of the Schwarzschild solution (as in any stationary black hole solution), it is the presence of the static (stationary) black hole that is responsible for the singularity of $i^{ \pm}$.

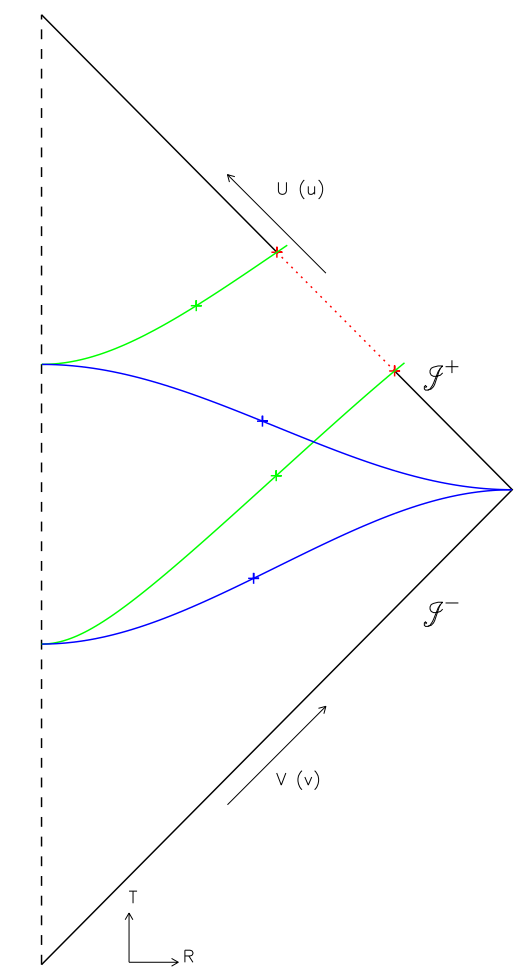

Figure 3: Spacelike hypersurfaces in the conformal picture.

Let us now assume that there is a particle that moves along the central world-line $r=0$, emitting radiation. For the sake of simplicity we assume that it emits electro-magnetic radiation, which travels along the outgoing null-cones to null-infinity. The null-cones are symbolized in Figure 2 by the straight lines going off the particle's world-line at $45^{\circ}$. We are now interested in the behaviour of the signal along various space-like hypersurfaces. In Figure 3 we show again the conformal diagram of Minkowski space. The generic features discussed below will be the same for any asymptotically flat space-time as long as we stay away from the corners of the diagram. The reason for choosing Minkowski space is simply one of convenience.

The vertical dashed line is the world-line of the particle that defines the time axis. We have displayed two asymptotically Euclidean space-like hypersurfaces intercepting the time axis at two different points and reaching out to space-like infinity. Furthermore, there are two hypersurfaces that intersect the time axis in the same two points as the asymptotically flat ones. They reach null-infinity, intersecting in a two-dimensional space-like surface. This geometric statement about the behaviour of the hypersurfaces in the unphysical space-time translates back to the physical space-time as a statement about asymptotic fall-off conditions of the induced (physical) metric on the hypersurfaces, namely that asymptotically the metric has constant negative curvature. This is, in particular, a property of the space-like hyperboloids in Minkowski space. Thus, such hypersurfaces are called hyperboloidal hypersurfaces. An important point to keep in mind is that the conformal space-time does not "stop" at $\mathscr{I}$ but that it can be extended smoothly beyond.

Living Reviews in Relativity

http: //www . livingreviews . org/Irr-2004-1/ 
The extension is not uniquely determined as we have already discussed in connection with the embedding of Minkowski space into the Einstein cylinder (cf. Figure 1). Thus, the extension plays no role in the concept of null-infinity but it can be very helpful for technical reasons, in particular when numerical issues are discussed.

We now imagine that the central particle radiates electro-magnetic waves of uniform frequency, i.e. proportional to $\sin (\omega \tau)$, where $\tau$ is the particle's proper time. This gives rise to a retarded electro-magnetic field on the entire space-time, which has the form $\phi \propto \sin (\omega u)$, where $u$ is a retarded time coordinate on Minkowski space with $u=\tau$ on the central world-line. We ignore the fall-off of the field because it is irrelevant for our present purposes. Let us now look at the waves on the various hypersurfaces.

In the physical space-time, the hypersurfaces extend to infinity, and we can follow the waves only up to an arbitrary but finite distance along the hypersurfaces. The end-points are indicated in Figure 3 as little crosses. The resulting waveforms are shown in Figure 4.
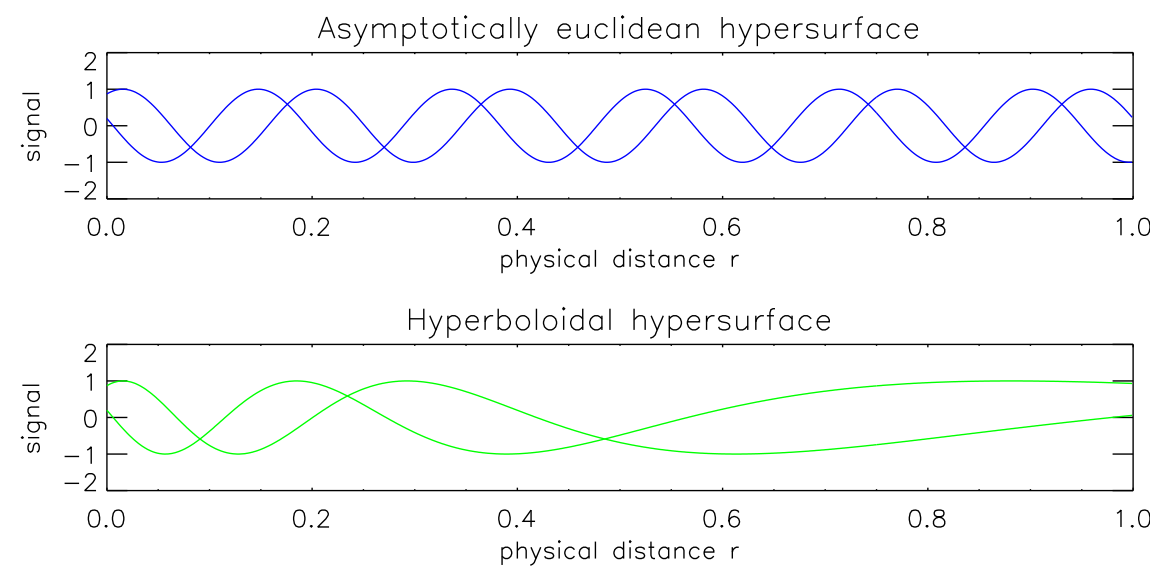

On $g$

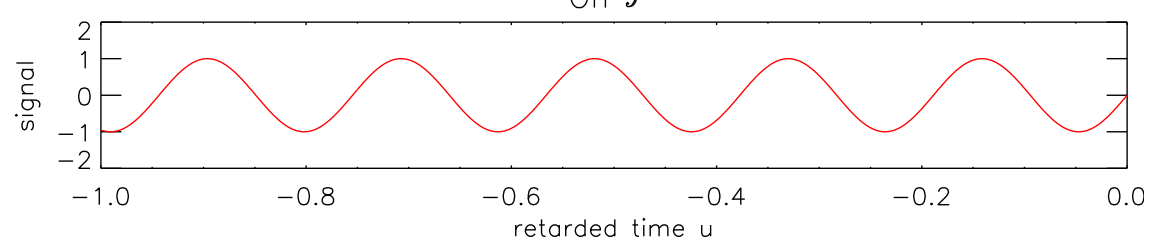

Figure 4: Waveforms in physical space-time.

The first diagram shows the situation on the asymptotically Euclidean surfaces. These are surfaces of constant Minkowski time, which implies that the signal is again a pure sine wave. Note, however, that this is only true for these special hypersurfaces. Even in Minkowski space-time we could choose space-like hypersurfaces that are not surfaces of constant Minkowski time but which nonetheless are asymptotically Euclidean. On such surfaces the wave would look completely different.

On the hyperboloidal surfaces the waves seem to "flatten out". The reason for the decrease in frequency is the fact that these surfaces tend to become more "characteristic" as they extend to infinity, thus approaching surfaces of constant phase of the retarded field.

The final diagram shows the signal obtained by an idealized observer who moves along the piece of $\mathscr{I}$ between the two intersection points with the hyperboloidal surfaces. The signal is recorded with respect to the retarded time $u$ which, in the present case, is a so-called Bondi parameter (see Section 4.3). Therefore, the observer measures a signal at a single frequency for a certain interval 
of this time parameter. A different Bondi time would result in a signal during a different time interval but with a single, appropriately scaled, frequency. Using an arbitrary time parameter would destroy the feature that only one frequency is present in the signal. This is, in fact, the only information that can be transmitted from the emitter to the receiver under the given circumstances.


Figure 5: Waveforms in conformal space-time.

The waveforms of the signal as they appear in the conformal space-time, i.e. with respect to a coordinate system that covers a neighbourhood of $\mathscr{I}$, are shown in Figure 5 . In the specific case of Minkowski space-time, we use the coordinates $T$ and $R$ on the Einstein cylinder. The signal on the asymptotically Euclidean surfaces shows the "piling up" of the waves as they approach space-like infinity. The signal on the hyperboloidal surfaces looks very similar to the physical case. Since the field and the surfaces are both smooth across $\mathscr{I}$, the signal can continue on across null-infinity without even noticing its presence. The points where $\mathscr{I}$ is crossed are indicated in the diagram as two little crosses. The values of the field at these points are the same as the boundary values of the signal in the third diagram. Here the signal in the same region of $\mathscr{I}$ as in Figure 4 is displayed, but with respect to the coordinate $U=\arctan u$ which is not a Bondi parameter. Accordingly, we see that the wavelength of the signal is not constant.

What these diagrams teach us is the following: It has been convenient in relativity to decompose space-time into space and time by slicing it with a family of space-like hypersurfaces. In most of the work on existence theorems of the Einstein equations it has been convenient to choose them to be Cauchy surfaces and thus asymptotically Euclidean. Also, in most numerical treatments of Einstein's equations the same method is used to evolve space-times from one space-like hypersurface to the next (see Section 4). Here the hypersurfaces used are finite because the numerical grids are necessarily finite. In the approaches based on the standard Einstein equations it makes no difference whether the grid is based on a finite portion of an asymptotically Euclidean or a hyperboloidal hypersurface. The fact that the space-time should be asymptotically flat has to be conveyed entirely by a suitable boundary condition, which has to be imposed at the boundary of the finite portion of the hypersurface (i.e. at the little crosses in Figure 3). However, this implies that the

Living Reviews in Relativity

http://www. livingreviews.org//rr-2004-1/ 
accuracy of the waveform templates obtained with such approaches depends to a large extent on the quality of that boundary condition. So far there exists no suitable boundary condition that would be physically reasonable and lead to stable codes.

In the conformal approach one has the option to "include infinity" by using the conformal field equations (see Section 3). Then the type of the space-like hypersurfaces becomes an issue. The diagrams show that the hyperboloidal surfaces are very well suited to deal with the radiation problems. They provide a foliation of the conformal space-time on which one can base the evolution with the conformal field equations. The solution obtained will be smooth near $\mathscr{I}$ and we "only" need to locate $\mathscr{I}$ on each hypersurface to read off the value of the radiation data (as indicated in the second diagram of Figure 5).

\subsection{Going further}

As we indicated already in the introduction, the amount of results and developments related to the conformal structure of space-times and, in particular, to conformal infinity is overwhelming. We take the opportunity to refer to several other developments, which are not treated here in detail.

There exist several reviews of the subject from different points of view, e.g. by Geroch [79], by Penrose [127], by Schmidt [150], by Newman and Tod [120], by Ashtekar [7, 8], and by Friedrich [64, $67]$.

A large part of the literature on null-infinity is concerned with "conserved quantities". There exist several ways to derive the Bondi-Sachs energy-momentum expression. It can be defined in terms of limits of integrals, called linkages [82], over spheres that approach a cut of null-infinity $\mathscr{I}^{+}$, where the integrals are taken over certain vector fields in the physical space-time that suitably approximate the infinitesimal generators of asymptotic symmetries. Penrose, who had earlier [128] re-expressed the original Bondi-Sachs expressions in terms of genuine geometric quantities at $\mathscr{I}^{+}$, has also derived them from his quasi-local mass proposal [131]. They can also be obtained by "helicity lowering" of the rescaled Weyl tensor at $\mathscr{I}^{+}$using a two-index asymptotic twistor [155]. Other approaches (see [85] for a review) start from a Hamiltonian or Lagrangian formulation of the theory and derive the energy-momentum expressions via Noether theorems or the momentmap of symplectic geometry (see e.g. [10, 13]). These formulations also provide a framework for "asymptotic quantization", a scheme that is geared towards a scattering-matrix description for gravity. The universal structure of $\mathscr{I}$ provides the necessary background structure for the definition of a phase-space of the radiative modes of the gravitational field and its subsequent quantization [9].

While the energy-momentum expressions all coincide, there is still disagreement about the various angular-momentum expressions (see e.g. the review article by Winicour [165]). This difficulty is caused by the group structure of the BMS group, which does not allow one to single out a unique Lorentz subgroup (it is obtained only as a factor group). Hopefully, these discrepancies will be resolved once the structure of the gravitational fields at $i^{0}$ is completely understood.

All the "conserved quantities" are associated with a (space-like) cut of null-infinity, which is used for evaluation of the surface integrals, and an infinitesimal generator of the asymptotic symmetry group used in defining the integrand. They are not conserved in a strict sense because they depend on the cut. The prime example is again the Bondi-Sachs energy-momentum, which obeys the famous Bondi-Sachs mass-loss formula (which relates the values of the energy-momentum at two given cuts with a negative definite "flux integral" over the part of $\mathscr{I}^{+}$between the two cuts).

Furthermore, there exist the somewhat mysterious Newman-Penrose constants [119], five complex quantities that are also defined by surface integrals over a cut of $\mathscr{I}^{+}$. In contrast to the previous conserved quantities, the NP constants are absolutely conserved in the sense that they do not depend on the particular cut used for the evaluation of the integrals. In space-times that have a regular point $i^{+}$, the NP constants turn out to be the value of the gravitational field at $i^{+}$. If 
$i^{+}$is singular, then the NP constants are still well-defined, although now they should probably be considered as the value of the gravitational field at an ideal point $i^{+}$. Other interpretations relate them to certain combinations of multipole moments of the gravitational field [119, 135]. People have tried to give an interpretation of the NP constants in terms of a Lagrangian or symplectic framework [84, 83, 139], but these results are still somewhat unsatisfactory. Very recently, Friedrich and Kánnár [71] were able to connect the NP constants defined at null-infinity to initial data on a space-like asymptotically Euclidean (time-symmetric) hypersurface.

Finally, we want to mention the recent formulation of general relativity as a theory of null hypersurfaces (see [105]). This theory has its roots in the observation that one can reconstruct the points of Minkowski space-time from structures defined on null-infinity. The future light cone emanating from an arbitrary point in Minkowski space-time is a shear-free null hypersurface intersecting $\mathscr{I}^{+}$in a cut. The shear-free property of the light cone translates into the fact that the cut itself is given as a solution of a certain differential equation, the "good cut equation" on $\mathscr{I}^{+}$. Conversely, it was realized that in flat space the solution space of the good cut equation is isometric to Minkowski space-time (in particular, it carries a flat metric). Attempts to generalize this property led to Newman's $\mathfrak{H}$-space construction [116], which associates with each (complexified) asymptotically flat and (anti-)self-dual space-time a certain complex four-dimensional manifold that carries a Ricci-flat metric. It is obtained as the solution space of the complex good cut equation. Trying to avoid the unphysical complexification has finally led to the above-mentioned null surface formulation of general relativity.

At this point the connection to Penrose's theory of twistors is closest. Newman's $\mathfrak{H}$-spaces were the motivation for the "non-linear graviton" construction [129], which associates with each antiself-dual vacuum space-time a certain three-dimensional complex manifold. The interpretation of these manifolds at the time was that they should provide the one-particle states of the gravitational field in a future quantum theory of gravity. For a recent review of twistor theory, we refer to [132]. The non-linear gravitons themselves have led to remarkable developments in pure mathematics (see e.g. the contributions in [96]).

Living Reviews in Relativity

http://www . livingreviews . org/lrr-2004-1/ 


\section{The Regular Conformal Field Equations}

It is clear that Penrose's method of conformally compactifying space-time does provide a convenient and elegant framework for discussing questions of asymptotics. What is not obvious, however, is how to answer the question of existence of space-times that actually do possess the asymptotic structure suggested by the conformal picture. On the one hand, a very specific geometric property of the space-time is required, namely the possibility to attach a smooth conformal boundary, and on the other hand, the Einstein equation, a differential equation on the metric of the space-time, has to be satisfied. It is not clear whether these two different requirements are indeed compatible.

To be more precise, one would like to know how many asymptotically flat solutions of the field equations actually exist, and whether this class of solutions contains the physically interesting ones that correspond to radiative isolated gravitating systems. The only possible avenue to answering questions of existence consists of setting up appropriate initial value problems and proving existence theorems for solutions of the Einstein equations subject to the appropriate boundary conditions.

The conventional Cauchy problem, which treats the Einstein equation as a second-order partial differential equation for the metric field, is already rather complicated by itself (see e.g. the review by Choquet-Bruhat and York [29]). But to obtain statements of the type mentioned above is further complicated by the fact that in order to discuss the asymptotic fall-off properties of solutions one would need to establish global (long time and large distance) existence together with detailed estimates about the fall-off behaviour of the solution.

The geometric characterization of the asymptotic conditions in terms of the conformal structure suggests that we should discuss the existence problem also in terms of the conformal structure. The general idea is as follows: Suppose we are given an asymptotically flat manifold, which we consider as being embedded into an appropriate conformally related unphysical space-time. The Einstein equations for the physical metric imply conditions for the unphysical metric and the conformal factor relating these two metrics. It turns out that one can write down equations that are regular on the entire unphysical manifold, even at those points that are at infinity with respect to the physical metric. Existence of solutions of these "regular conformal field equations" on the conformal manifold then translate back to (semi-)global results for asymptotically flat solutions of the field equations in physical space. This approach towards the existence problem has been the programme followed by Friedrich since the late 1970's.

In the remainder of this section we will discuss the conformal field equations, which were derived by Friedrich, and the various subproblems that have been successfully treated using the conformal field equations.

\subsection{General properties of the conformal field equations}

Before deriving the equations we need to define the arena where the discussion is taking place.

Definition 2: A conformal space-time is a triple $\left(\mathcal{M}, g_{a b}, \Omega\right)$ such that

(1) $\left(\mathcal{M}, g_{a b}\right)$ is a (time- and space-orientable) Lorentz manifold;

(2) $\Omega$ is a smooth scalar field on $\mathcal{M}$ such that the set $\widetilde{\mathcal{M}}=\{p \in \mathcal{M}: \Omega(p)>0\}$ is non-empty and connected;

(3) the gravitational field $K^{a}{ }_{b c d}=\Omega^{-1} C^{a}{ }_{b c d}$ extends smoothly to all of $\mathcal{M}$.

Two conformal space-times $\left(\mathcal{M}, g_{a b}, \Omega\right)$ and $\left(\hat{\mathcal{M}}, \hat{g}_{a b}, \hat{\Omega}\right)$ are equivalent if $\mathcal{M}$ and $\hat{\mathcal{M}}$ are diffeomorphic and if, after identification of $\mathcal{M}$ and $\hat{\mathcal{M}}$ with a suitable diffeomorphism, there exists a strictly positive scalar field $\theta$ on $\mathcal{M}$ such that $\hat{\Omega}=\theta \Omega$ and $\hat{g}_{a b}=\theta^{2} g_{a b}$. 
From this definition follows that $\widetilde{\mathcal{M}}$ is an open sub-manifold of $\mathcal{M}$ on which a metric $\tilde{g}_{a b}=$ $\Omega^{-2} g_{a b}$ is defined, which is invariant in the sense that two equivalent conformal space-times define the same metric $\tilde{g}_{a b}$.

The space-time $\left(\widetilde{\mathcal{M}}, \tilde{g}_{a b}\right)$ allows the attachment of a conformal boundary, which is given by $\mathscr{I}=\{p \in \mathcal{M}: \Omega(p)=0, d \Omega \neq 0\}$. The above definition of conformal space-times admits much more general situations than those arising from asymptotically flat space-times; this generality is sometimes needed for numerical purposes.

Under the conditions of Definition 2, it follows that the Weyl tensor vanishes on $\mathscr{I}$ because the gravitational field (i.e. the rescaled Weyl tensor) is smooth on $\mathcal{M}$. Note that we make no assumptions about the topology of $\mathscr{I}$. If each null geodesic which starts from the inside of $\widetilde{\mathcal{M}}$ has a future and a past endpoint on $\mathscr{I}$, then $\widetilde{\mathcal{M}}$ is asymptotically simple in the sense of Definition 1 . If, in addition, the metric $\tilde{g}_{a b}$ is a vacuum metric then $\mathscr{I}$ has the implied topology $S^{2} \times \mathbb{R}$. Note also that it is quite possible to have situations where $\tilde{g}_{a b}$ is a vacuum metric and where the topology of $\mathscr{I}$ is not $S^{2} \times \mathbb{R}$, but e.g. $T^{2} \times \mathbb{R}$. Then, necessarily, there must exist null geodesics that do not reach $\mathscr{I}$.

In the special case when $\mathscr{I}$ is empty, the conformal factor $\Omega$ is strictly positive, i.e. $\widetilde{\mathcal{M}}=\mathcal{M}$, and the conformal space-time is isometric to the physical space-time ( choosing $\theta=\Omega^{-1}$ ).

Our goal is to express the vacuum equations in $\widetilde{\mathcal{M}}$ in terms of geometric quantities on the unphysical space-time. Consider first the Einstein vacuum equation for the metric $\tilde{g}_{a b}=\Omega^{2} g_{a b}$. When expressed in terms of unphysical quantities it reads (see the formulae of Appendix 7)

$$
0=\tilde{G}_{a b}=G_{a b}-\frac{2}{\Omega}\left(\nabla_{a} \nabla_{b} \Omega-g_{a b} \square \Omega\right)-\frac{3}{\Omega^{2}} g_{a b} \nabla_{c} \Omega \nabla^{c} \Omega .
$$

This equation can be interpreted as the Einstein equation for the metric $g_{a b}$ with a source term which is determined by the conformal factor. If we assume $\Omega$ to be known, then it is a second order equation for $g_{a b}$, which is formally singular on $\mathscr{I}$, where $\Omega$ vanishes. Therefore, it is very hard to make any progress towards the existence problem using this equation. To remedy this situation, Friedrich [54, 55, 56] suggested to consider a different system of equations on $\mathcal{M}$ that can be derived from the geometric structure on $\mathcal{M}$, the conformal transformation properties of the curvature, and the vacuum Einstein equation on $\widetilde{\mathcal{M}}$. It consists of equations for a connection $\nabla_{a}$, its curvature, and certain other fields obtained from the curvature and the conformal factor.

Let us assume that $\nabla_{a}$ is a connection on $\mathcal{M}$ that is compatible with the metric $g_{a b}$ so that

$$
\nabla_{c} g_{a b}=0
$$

holds. This condition does not fix the connection. Let $T_{b c}^{a}$ and $R_{b c d}^{a}$ denote the torsion and curvature tensors of $\nabla_{a}$. We will write down equations for the following unknowns:

- the connection $\nabla_{a}$,

- the conformal factor $\Omega$, a one-form $\Sigma_{a}$, and a scalar function $S$,

- a symmetric trace-free tensorfield $\Phi_{a b}$, and

- a completely trace-free tensorfield $K^{a}{ }_{b c d}$ that has the symmetries of the Weyl tensor.

We introduce the zero-quantity

$$
Z=\left(T_{b c}^{a}, \mathcal{Q}_{a b c d}, \mathcal{P}_{a b c}, \mathcal{B}_{b c d}, \mathcal{S}_{a}, \mathcal{S}_{a b}, \mathcal{D}_{a}\right),
$$

where $T_{b c}^{a}$ is the torsion tensor of $\nabla_{a}$, and the other components of $Z$ are defined in terms of the unknowns by

$$
\mathcal{Q}_{a b c d} \equiv R_{a b c d}-\Omega K_{a b c d}+2 g_{c[a} \Phi_{b] d}-2 g_{d[a} \Phi_{b] c}-4 g_{c[a} g_{b] d} \Lambda
$$

Living Reviews in Relativity

http://www . livingreviews . org//rr-2004-1/ 


$$
\begin{aligned}
\mathcal{P}_{a b c} & \equiv 2 \nabla_{[c} \Phi_{a] b}+2 g_{b[c} \nabla_{a]} \Lambda-K_{c a b}{ }^{d} \Sigma_{d} \\
\mathcal{B}_{b c d} & \equiv \nabla_{a} K_{b c d}^{a}, \\
\mathcal{S}_{a} & \equiv \nabla_{a} \Omega-\Sigma_{a} \\
\mathcal{S}_{a b} & \equiv \nabla_{a} \Sigma_{b}-g_{a b} S+\Omega \Phi_{a b} \\
\mathcal{D}_{a} & \equiv \nabla_{a} S+\Phi_{a b} \Sigma^{b}-\Omega \nabla_{a} \Lambda-2 \Lambda \Sigma_{a}
\end{aligned}
$$

In addition, we consider the scalar field

$$
\mathcal{T} \equiv 2 \Omega S-2 \Omega^{2} \Lambda-\Sigma_{a} \Sigma^{a}
$$

on $\mathcal{M}$. The equations $Z=0$ are the regular conformal vacuum field equations. They are first order equations. In contrast to Equation (13), this system is regular ${ }^{1}$ on $\mathcal{M}$, even on $\mathscr{I}$ because there are no terms containing $\Omega^{-1}$.

Consider the equation $\mathcal{B}_{b c d}=\nabla_{a} K_{b c d}^{a}=0$. This subsystem lies at the heart of the full system of conformal field equations because it feeds back into all the other parts. It was pointed out in Section 2.2 that the importance of the Bianchi identity had been realized by Sachs. However, it was first used in connection with uniqueness and existence proofs only by Friedrich [56, 55]. Its importance lies in the fact that it splits naturally into a symmetric hyperbolic system of evolution equations $^{2}$ and constraint equations. Energy estimates for the symmetric hyperbolic system naturally involve integrals over a certain component of the Bel-Robinson tensor [63], a well-known tensor in general relativity that has certain positivity properties.

The usefulness of the conformal field equations is documented in

Theorem 1: Suppose that $\nabla_{a}$ is compatible with $g_{a b}$ and that $Z=0$ on $\mathcal{M}$. If $\mathcal{T}=0$ at one point of $\mathcal{M}$, then $\mathcal{T}=0$ everywhere and, furthermore, the metric $\Omega^{-2} g_{a b}$ is a vacuum metric on $\widetilde{\mathcal{M}}$.

Proof: From the vanishing of the torsion tensor it follows that $\nabla_{a}$ is the Levi-Civita connection for the metric $g_{a b}$. Then, $\mathcal{Q}_{a b c d}=0$ is the decomposition of the Riemann tensor into its irreducible parts which implies that the Weyl tensor $C^{a}{ }_{b c d}=\Omega K^{a}{ }_{b c d}$, that $\Phi_{a b}$ is the trace-free part of the Ricci tensor, and that $\Lambda=\frac{1}{24} R$. The equation $\mathcal{S}_{a}=0$ defines $\Sigma_{a}$ in terms of $\Omega$, and the trace of the equation $\mathcal{S}_{a b}=0$ defines $S=\frac{1}{4} \square \Omega$. The trace-free part of that equation is the statement that $\tilde{\Phi}_{a b}=0$, which follows from the conformal transformation property (113) of the trace-free Ricci tensor. With these identifications the equations $\mathcal{B}_{a b c}=0$ respectively $\mathcal{P}_{a b c}=0$ do not yield any further information because they are identically satisfied as a consequence of the Bianchi identity on $(\mathcal{M}, g)$, respectively $(\widetilde{\mathcal{M}}, \tilde{g})$.

Finally, we consider the field $\mathcal{T}$. Taking its derivative and using $\mathcal{S}_{a b}=0$ and $\mathcal{D}_{a}=0$, we obtain $\nabla_{a} \mathcal{T}=0$. Hence, $\mathcal{T}$ vanishes everywhere if it vanishes at one point. It follows from the transformation (114) of the scalar curvature under conformal rescalings that $\mathcal{T}=0$ implies $\tilde{\Lambda}=0$. Thus, $\tilde{g}_{a b}$ is a vacuum metric.

\footnotetext{
${ }^{1}$ It might be worthwhile to point out that the notion of regularity of the system of equations has nothing whatsoever to do with the regularity of its solutions. While the former is a formal property of the system which can be established simply by inspection, the latter is usually rather difficult to establish because it involves detailed investigations of the appropriate well-posed problems.

${ }^{2} \mathrm{~A}$ system of partial differential equations for unknowns $\mathbf{u}=\left(u^{1}, \ldots, u^{M}\right)^{t}$ on $\mathbb{R}^{N+1}$ of the form

$$
\mathbf{A}^{0} \partial_{0} \mathbf{u}+\sum_{k=1}^{N} \mathbf{A}^{k} \partial_{k} \mathbf{u}=\mathbf{b}
$$

is called symmetric hyperbolic, if the matrices $\mathbf{A}^{k}, k=0, \ldots, N$, are symmetric and the matrix $\mathbf{A}^{0}$ is positive definite. Both the matrices and the right hand side $\mathbf{b}$ may depend on the coordinates $x^{0}, x^{1}, \ldots, x^{N}$ and the unknowns $\mathbf{u}$.
} 
It is easy to see that the conformal field equations are invariant under the conformal rescalings of the metric specified in Definition 2 and the implied transformation of the unknowns. The conformal invariance of the system implies that the information it contains depends only on the equivalence class of the conformal space-time.

The reason for the vanishing of the gradient of $\mathcal{T}$ is essentially this: If we impose the equation $\tilde{\Phi}_{a b}=0$ for the trace-free part of the Ricci tensor of a manifold, then by use of the contracted Bianchi identity we obtain $\tilde{\nabla}_{a} \tilde{\Lambda}=0$. Expressing this in terms of unphysical quantities leads to the reasoning in Theorem 1 . The special case $\Omega=1$ reduces to the standard vacuum Einstein equations, because then we have $K_{a b c d}=C_{a b c d}$ and $\Sigma_{a}=0$. Then $\mathcal{S}_{a b}=0$ implies $\Phi_{a b}=0$ and $S=0$, while $\mathcal{T}=0$ forces $\Lambda=0$. The other equations are identically satisfied.

Given a smooth solution of the conformal field equations on a conformal manifold, Theorem 1 implies that on $\widetilde{\mathcal{M}}$ we obtain a solution of the vacuum Einstein equation. In particular, since the Weyl tensor of $g_{a b}$ vanishes on $\mathscr{I}$ due to the smoothness of the gravitational field, this implies that the Weyl tensor has the peeling property in the physical space-time. Therefore, if existence of suitable solutions of the conformal field equations on a conformal manifold can be established, one has automatically shown existence of asymptotically flat solutions of the Einstein equations. The main advantage of this approach is the fact that the conformal compactification supports the translation of global problems into local ones.

Note that the use of the conformal field equations is not limited to vacuum space-times. It is possible to include matter fields into the conformal field equations provided the equations for the matter have well-defined and compatible conformal transformation properties. This will be the case for most of the interesting fundamental field equations (Maxwell, Yang-Mills [63], scalar wave $[90,91]$, etc.).

\subsection{The reduction process for the conformal field equations}

We have set up the conformal field equations as a system of equations for the conformal geometry of a conformal Lorentz manifold. As such they are invariant under general diffeomorphisms and, as we have seen, under conformal rescalings of the metric. In this form they are not yet very useful for treating questions of existence of solutions or even for numerical purposes. For existence results and numerical evolution, the geometric equations have to be transformed into partial differential equations for tensor components, which can then be used to set up well-posed initial value problems for hyperbolic systems of evolution equations.

This process, sometimes referred to as "hyperbolic reduction" consists of several steps. First, one needs to break the invariance of the equations. By imposing suitable gauge conditions one can specify a coordinate system, a linear reference frame, and a conformal factor. Then, the equations can be written as equations for the components of the geometric quantities with respect to the chosen frame in the chosen conformal gauge and as functions of the chosen coordinates. In the next step, one needs to extract from the equations a subsystem of propagation equations that is hyperbolic so that it has a well-posed initial value problem. It is often referred to as the "reduced equations". Finally, one has to make sure that solutions of the reduced system give rise to solutions of the full system. This step may involve the verification that the gauge conditions imposed are compatible with the propagation equations, or that other equations (constraints) not included in the reduced system are preserved under the propagation. The first two steps, choice of gauge and extraction of the reduced system, are very much related. Gauge conditions should be imposed such that they lead to a hyperbolic reduced system. Furthermore, the gauge conditions should be such that they can be imposed locally without loss of generality.

The gauge freedom present in the conformal field equations can easily be determined. The freedom to choose the coordinates amounts to four scalar functions while the linear reference frame, which we take to be orthogonal, can be specified by a Lorentz rotation, which amounts

Living Reviews in Relativity

http: //www. livingreviews.org/Irr-2004-1/ 
to six free functions. Finally, the choice of a conformal factor contributes another free function. Altogether, there are eleven functions that can be chosen at will.

Once the geometric equations have been transformed into equations for components, the next step is to extract the reduced system. These are equations for the components of the geometric quantities defined above as well as for gauge-dependent quantities: the components of the frame with respect to the coordinate basis, the components of the connection with respect to the given frame, and the conformal factor.

There are several well-known choices for coordinates (harmonic, Gauß, Bondi, etc.), as well as for frames (Fermi-Walker transport, Newman-Penrose, etc.). These are usually "hard-wired" into the equations and one has no further control on the properties of the gauge. Gauß coordinates for instance have the tendency to become singular when the geodesic congruence that is used for their definition starts to self-intersect. Similarly, Bondi coordinates are attached to null-hypersurfaces, which have the tendency to self-intersect, thus destroying the coordinate system. In the context of existence proofs and the numerical evolution of the equations, it is of considerable interest to have additional flexibility in order to prevent the coordinates or the frame from becoming singular. The goal is to "fix the gauge" in as flexible a manner as possible, and to obtain reduced equations that still have useful properties.

A scheme to obtain the reduced equations in symmetric hyperbolic form while still allowing for arbitrary gauges has been devised by Friedrich [59] (see also [66] for various examples). The idea is based on the following observation. Cartan's structure equations, which express the torsion and curvature tensors in terms of tetrad and connection coefficients, are two-form equations: They are skew on two indices, and the information contained in the equations is not enough to fix the tetrad and the connection by specifying the torsion and the curvature. The additional information is provided by fixing a gauge. Normally, this is achieved by reducing the number of variables, in this case the number of tetrad components and connection coefficients. However, one can just as well add appropriate further equations to have enough equations for all unknowns. The additional equations should be chosen so that the ensuing system has "nice" properties.

We illustrate this procedure by a somewhat trivial example. Consider, in flat space with coordinates $\left(x^{\mu}\right)=\left(t, x^{1}, x^{2}, x^{3}\right)$, a one-form $\omega$ which we require to be closed:

$$
\partial_{\mu} \omega_{\nu}-\partial_{\nu} \omega_{\mu}=0 .
$$

From this equation we can extract three evolution equations, namely

$$
\begin{aligned}
& \partial_{t} \omega_{1}-\partial_{1} \omega_{0}=0, \\
& \partial_{t} \omega_{2}-\partial_{2} \omega_{0}=0, \\
& \partial_{t} \omega_{3}-\partial_{3} \omega_{0}=0 .
\end{aligned}
$$

Obviously, these three equations are not sufficient to reconstruct $\omega$ from appropriate initial data. One possibility to proceed from here is to specify one component of $\omega$ freely and then obtain equations for the other three. However, it is easily seen that only by specifying $\omega_{0}$ can we achieve a pure evolution system. Otherwise, we get mixtures of evolution and constraint equations. So we may note that proceeding in this way leads to a restriction of possibilities as to which components should be specified freely and, in general, it also entails that derivatives of the specified component appear.

Another possible procedure is to enlarge the system by adding an equation for the time derivative of $\omega_{0}$. Doing this covariantly implies that we should add an equation in the form of a divergence

$$
\partial^{\mu} \omega_{\mu}=F,
$$

where $F$ is an arbitrary function. This results in the system

$$
\partial_{t} \omega_{0}-\partial_{1} \omega_{1}-\partial_{2} \omega_{2}-\partial_{3} \omega_{3}=F,
$$




$$
\begin{aligned}
& \partial_{t} \omega_{1}-\partial_{1} \omega_{0}=0, \\
& \partial_{t} \omega_{2}-\partial_{2} \omega_{0}=0, \\
& \partial_{t} \omega_{3}-\partial_{3} \omega_{0}=0,
\end{aligned}
$$

which is symmetric hyperbolic for any choice of $F$. Note also that $F$ appears as a source term and only in undifferentiated form. Clearly, our influence on the component $\omega_{0}$ is now very indirect via the solution of the system, while before we could specify it directly.

In a similar way, one proceeds in the present case of the conformal field equations. Note, however, that this way of fixing a gauge is not at all specific to these equations. Since it depends essentially only on the form of Cartan's structure equations it is applicable in all cases where these are part of the first order system. The Cartan equations can be regarded as exterior equations for the one-forms $\omega_{a}^{\mu}$ dual to a tetrad $e_{\mu}^{a}$ and the connection one-forms $\omega_{a \nu}^{\mu}=\omega_{c}^{\mu} \nabla_{a} e_{\nu}^{c}$. Similar to the system above, the equations involve only the exterior derivative of the one-forms, and so we expect that we should add equations in divergence form, namely

$$
\begin{aligned}
\nabla^{a} \omega_{a}^{\mu} & =F^{\mu}, \\
\nabla^{a} \omega_{a \nu}^{\mu} & =F^{\mu}{ }_{\nu},
\end{aligned}
$$

with arbitrary gauge source functions $F^{\mu}$ for fixing coordinates and $F^{\mu}{ }_{\nu}$ for choosing a tetrad. Note that $\omega_{a}^{\mu}=\nabla_{a} x^{\mu}$ implies $F^{\mu}=\square x^{\mu}$.

In a given gauge (i.e. coordinates and frame field are specified) the gauge sources can be determined from

$$
\begin{aligned}
F^{\mu} & =\square x^{\mu}, \\
F^{\mu}{ }_{\nu} & =\nabla^{a}\left(\omega_{c}^{\mu} \nabla_{a} e_{\nu}^{c}\right) .
\end{aligned}
$$

In fact, these equations are exactly the same equations as $(23,24)$ except that they are written in a more invariant form. Now it is obvious that the gauge sources contain information about the coordinates and the frame used. What needs to be shown is that any specification of the gauge sources fixes a gauge. In fact, suppose we are given functions $\hat{F}^{\mu}$ and $\hat{F}^{\mu}{ }_{\nu}$ on $\mathbb{R}^{4}$; then there exist (locally) coordinates $\hat{x}^{\mu}$ and a frame $\hat{e}_{\mu}^{a}$ so that in that coordinate system the gauge sources are just the prescribed functions $\hat{F}^{\mu}$ and $\hat{F}^{\mu}{ }_{\nu}$. This follows from the equations

$$
\begin{aligned}
\square \hat{x}^{\mu} & =\hat{F}^{\mu}\left(\hat{x}^{\nu}\right), \\
\nabla^{a}\left(\hat{\omega}_{c}^{\mu} \nabla_{a} \hat{e}_{\nu}^{c}\right) & =\hat{F}^{\mu}{ }_{\nu}\left(\hat{x}^{\rho}\right) .
\end{aligned}
$$

These are semi-linear wave equations which determine a unique solution from suitably given initial data close to the initial surface. Note that on the right hand side of (27) there is a function of the $\hat{x}^{\mu}$, and not a source term. The equations can be solved in steps. Once the coordinates $\hat{x}^{\mu}$ have been determined from (27), the right hand side of (28) can be considered as a source term.

Finally, we need to discuss the gauge freedom in the choice of the conformal factor $\Omega$. In many discussions of asymptotic structure, the conformal factor is chosen in such a way that null-infinity is divergence-free, in addition to the vanishing of its shear, which is a consequence of the asymptotic vacuum equations. That means that infinitesimal area elements remain unchanged in size as they are parallelly transported along the generators of $\mathscr{I}$. Since they also remain unchanged in form due to the vanishing shear of $\mathscr{I}$, they remain invariant and hence they can be used to define a unique metric on the space of generators of $\mathscr{I}$. This choice simplifies many calculations on $\mathscr{I}$, still leaving the conformal factor quite arbitrary away from $\mathscr{I}$. Yet, in numerical applications this choice of the conformal factor may be too rigid and so one needs a flexible method for fixing the conformal factor.

Living Reviews in Relativity

http: //www. livingreviews.org//rr-2004-1/ 
It turns out that one can introduce a gauge source function for the conformal gauge as well. Consider the change of the scalar curvature under the conformal rescaling $g_{a b} \mapsto \hat{g}_{a b}=\theta^{2} g_{a b}$, $\Omega \mapsto \hat{\Omega}=\theta \Omega$ : It transforms according to

$$
\Lambda \mapsto \hat{\Lambda}=\theta^{-2}\left(\Lambda+\frac{\square \theta}{4 \theta}\right) .
$$

Reading this transformation law as an equation for $\theta$ we obtain

$$
\square \theta=4 \theta\left(\theta^{2} \hat{\Lambda}-\Lambda\right) .
$$

It follows from this equation that we may regard the scalar curvature as a gauge source function for the conformal factor: Suppose we specify the function $\hat{\Lambda}$ arbitrarily on $\mathcal{M}$, then Equation (29) is a non-linear wave equation for $\theta$ which can be solved given suitable initial data. This determines a unique $\theta$, hence a unique $\hat{\Omega}$ and $\hat{g}_{a b}$ such that the scalar curvature of the rescaled metric $\hat{g}_{a b}$ has scalar curvature $\hat{\Lambda}$. Note that these considerations are local. They show that locally the gauges can be fixed arbitrarily. However, the problem of identifying and fixing a gauge globally is very difficult but also very important, because only when the gauges are globally known can one really compare two different space-times.

Having established that the gauge sources do, in fact, fix a unique gauge locally, we can now split the system of conformal field equations into evolution equations and constraints. The resulting system of equations is exhibited below. The reduction process is rather straightforward but tedious. It is sketched in Appendix 6. Here, we only describe it very briefly. We introduce an arbitrary time-like unit vector field $t^{a}$, which has a priori no relation to the tetrad field used for framing. We split all the tensorial quantities into the parts that are parallel and orthogonal to that vector field using the projector $h_{a}{ }^{b}=\delta_{a}^{b}-t_{a} t^{b}$. The connection coefficients for the four-dimensional connection $\nabla_{a}$ are treated differently. We introduce the covariant derivatives of the vector field $t^{a}$ by

$$
\chi_{b}^{a}=h_{b}^{c} \nabla_{c} t^{a}, \quad \chi^{a}=t^{c} \nabla_{c} t^{a} .
$$

They account for half $(9+3)$ of the four-dimensional connection coefficients. The other half is captured by defining a covariant derivative $\partial_{a}$ that has the property that it annihilates both $t^{a}$ and $t_{a}$ and agrees with $\nabla_{a}$ when acting on tensors orthogonal to $t^{a}$ (see Equations (42)). Note that we have not required that $t^{a}$ be the time-like member of the frame, nor have we assumed that it be hypersurface orthogonal. In the latter case, $\chi_{a b}$ is the extrinsic curvature of the family of hypersurfaces orthogonal to $t^{a}$ and hence it is symmetric. Furthermore, the derivative $\partial_{a}$ agrees with the Levi-Civita connection of the metric $h_{a b}$ induced on the leaves by the metric $g_{a b}$.

We write the equations in terms of the derivative $\partial_{a}$ and the "time derivative" $\partial$, which is defined in a way similar to $\partial_{a}$ (see Equation (42)), because in this form it is quite easy to see the symmetric hyperbolicity of the equations.

As they stand, the Equations $(93,94,95,96,97,98,99,100,101,102,103,104,105,106)$ form a symmetric hyperbolic system of evolution equations for the collection of 65 unknowns

$$
\left\{c^{\mu}, c_{a}^{\mu}, \chi_{b}^{a}, \chi^{a}, \Gamma_{a}{ }^{b} c, \Lambda_{b}^{a}, \phi_{a b}, \phi_{a}, E_{a b}, B_{a b}, \Omega, \sigma_{a}, \sigma, S\right\} .
$$

This property is present irrespective of the particular gauge. For any choice of the gauge source functions $F^{\mu}, F_{a}, F_{a b}$, and $\Lambda$, the system is symmetric hyperbolic. The fact that the gauge sources appear only in undifferentiated form implies that one can specify them not only as functions of the space-time coordinates but also as functions of the unknown fields. In this way, one can feed information about the current status of the evolution back into the system in order to influence the future development. 
Other ways of specifying the coordinate gauge, including the familiar choice of a lapse function and a shift vector, are not as flexible because then not only do these functions themselves appear in the equations, but so do their derivatives. Specifying them as functions of the unknown fields alters the principal part of the system and, hence, the propagation properties of the solution. This may not only corrupt the character of the system but it may also be disastrous for numerical applications, because an uncontrolled change of the local propagation speeds implies that the stability of a numerical scheme can break down due to violation of the CFL condition (see [46] for a more detailed discussion of these issues). However, due to the intuitive meaning of lapse and shift they are used (almost exclusively) in numerical codes.

There are several other ways to write the equations. Apart from various possibilities to specify the gauges that result in different systems with different numbers of unknowns, one can also set up the equations using spinorial methods. This was the method of choice in almost all of Friedrich's work (see e.g. [63] and also [45]). The ensuing system of equations is analogous to those obtained here using the tetrad formalism. The main advantages of using spinors is the fact that the reduction process automatically leads to a symmetric hyperbolic system, that the variables are components of irreducible spinors which allows for the elimination of redundancies, and that variables and equations become complex and hence easier to handle.

Another possibility is to ignore the tetrad formalism altogether (or, more correctly, to choose as a basis for the tangent spaces the natural coordinate frame). This also results in a symmetric hyperbolic system of equations (see [66, 94]), in which the gauge-dependent variables are not the frame components with their corresponding connection coefficients, but the components of the spatial metric together with the usual Christoffel symbols and the extrinsic curvature.

The fact that the reduced equations form a symmetric hyperbolic system leads, via standard theorems, to the existence of smooth solutions that evolve uniquely from suitable smooth data given on an initial surface. We have the

Theorem 2 (Friedrich [59]): For functions $F^{\mu}, F_{a}, F_{a b}, \Lambda$ on $\mathbb{R}^{4}$ and data given on some initial surface, let $u$ be the solution of the reduced equations. If u satisfies the conformal field equations (16, 17, 18, 19, 20, 21) on the initial surface, then, in fact, it satisfies them on the entire domain of dependence of the initial surface in the space-time defined by $u$.

The proof of this theorem relies on the existence of a "subsidiary system" of equations for the zero-quantity $Z$ (see Equation (15)), whose vanishing indicates the validity of the conformal field equations. This system turns out to be linear, symmetric hyperbolic, and homogeneous. Thus, one has uniqueness of the solutions so that $Z$ vanishes in the domain of dependence of the initial surface if it vanishes on the surface. Hence, the conformal field equations hold. It can be shown that solutions obtained from different gauge source functions are in the same conformal class, so they lead to the same physical space-time.

\subsection{Initial value problems}

From the physical point of view the most interesting scenario is the following one: A gravitating material system (e.g. several extended bodies) evolves from a given initial state, possibly interacting with incoming gravitational radiation and emitting outgoing gravitational radiation until it reaches a final state. This situation is sketched in Figure 6.

In more mathematical terms, this requires the solution of an initial value problem: We provide appropriate initial data, describing the initial configuration of the matter and the geometry, on a hyperboloidal hypersurface $\Sigma_{0}$, and appropriate boundary data, describing the incoming gravitational radiation, on the piece of $\mathscr{I}^{-}$that is in the future of $\Sigma_{0}$. Then we have to show that there is a unique solution of the conformal field equations coupled to the matter equations that exists for

Living Reviews in Relativity

http: //www. livingreviews.org/Irr-2004-1/ 


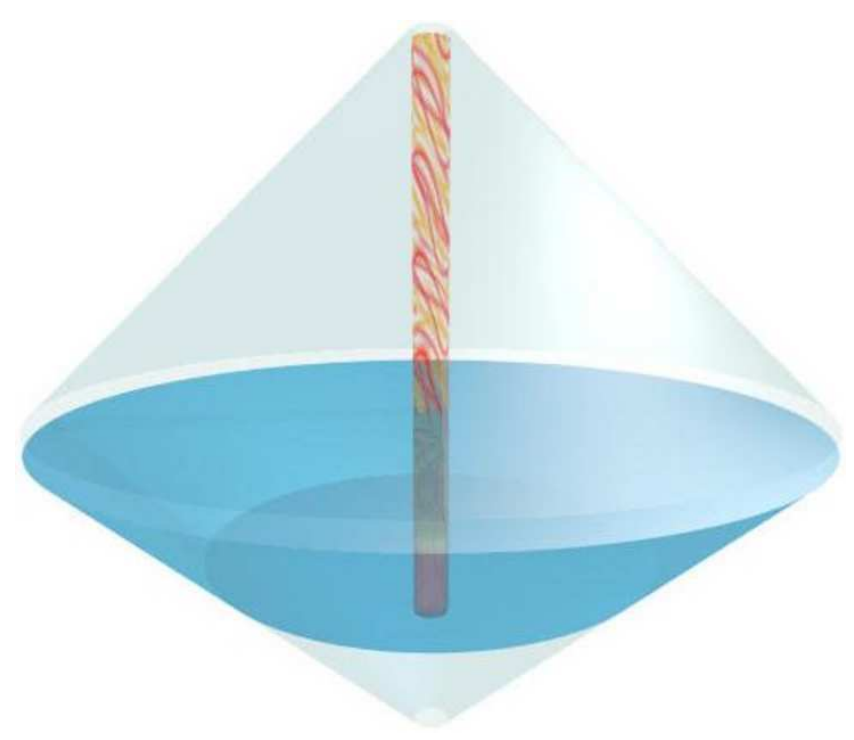

Figure 6: The physical scenario: The figure describes the geometry of an isolated system. Initial data are prescribed on the blue parts, i.e. on a hyperboloidal hypersurface and the part of $\mathscr{I}^{-}$ which is in its future. Note that the two cones $\mathscr{I}^{+}$and $\mathscr{I}^{-}$are separated to indicate the nontrivial transition between them.

some time. If the situation is "close enough" to a Newtonian situation, i.e. the gravitational waves are weak and the matter itself is rather "tame", then one would expect that there is a solution, i.e. a space-time, that is regular on arbitrary hyperboloidal hypersurfaces intersecting $\mathscr{I}^{+}$. In general, however, we cannot expect to have a regular point $i^{+}$representing time-like infinity.

So far, results of this kind are out of reach. The reason is not so much the incorporation of matter into the conformal field equations but a more fundamental one. Space-like infinity $i^{0}$ is a singularity for the conformal structure of any space-time that has a non-vanishing ADM mass. Without the proper understanding of $i^{0}$ there will be no way to bridge the gap between past and future null-infinity, because $i^{0}$ provides the link between the incoming and the outgoing radiation fields.

The results obtained so far are concerned only with the pure radiation problem, i.e. the vacuum case. In [31] Christodoulou and Klainerman prove the global non-linear stability of Minkowski space, i.e. the existence of global solutions of the Einstein vacuum equations for "small enough" Cauchy data that satisfy certain fall-off conditions at space-like infinity. Their result qualitatively confirms the expectations based on the concept of asymptotic flatness. However, they do not recover the peeling property for the Weyl tensor but a weaker fall-off, which implies that in this class of solutions the conformal compactification would not be as smooth as it was expected to be. This raises the question whether their results are sharp, i.e. whether there are solutions in this class that indeed have their fall-off behaviour. In that case, one would probably have to strengthen the fall-off conditions of the initial data at space-like infinity in order to establish the correct peeling of the Weyl tensor. Then, an interesting question arises as to what the physical meaning of these stronger fall-off conditions is. An indication that maybe more restrictive conditions are needed is provided by the analysis of the initial data on hyperboloidal hypersurfaces (see below).

The first result [57] obtained with the conformal field equations is concerned with the asymptotic characteristic initial value problem (see Figure 7 ) in the analytic case. It was later generalized to the $\mathcal{C}^{\infty}$ case. 


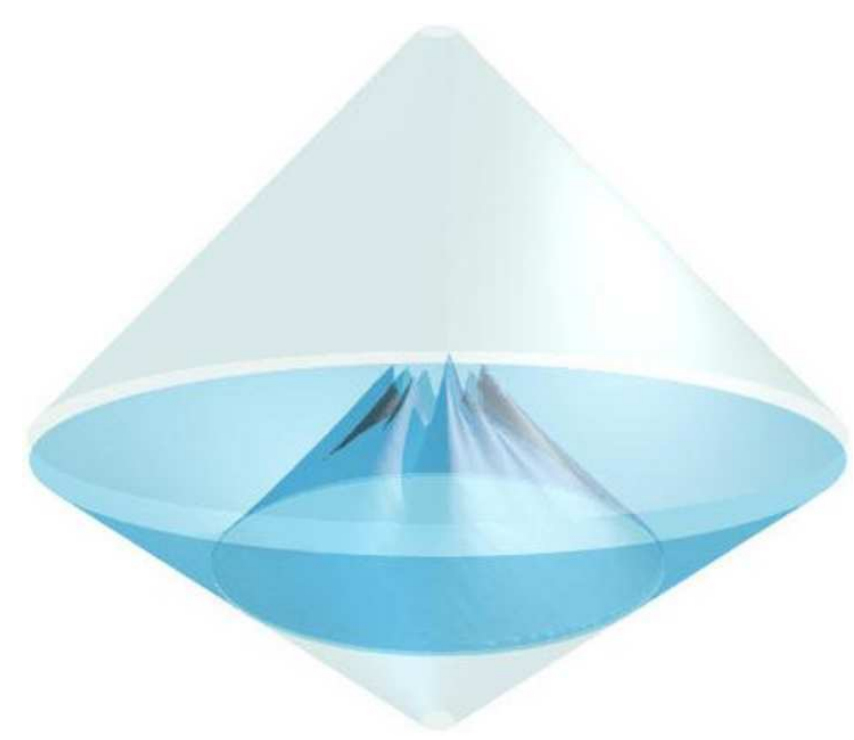

Figure 7: The geometry of the asymptotic characteristic initial value problem: Characteristic data are given on the blue parts, i.e. an ingoing null surface and the part of $\mathscr{I}^{-}$that is in its future. Note that the ingoing surface may develop self-intersections and caustics.

In this kind of initial value problem, one specifies data on an ingoing null hypersurface $\mathcal{N}$ and that part of $\mathscr{I}$ that is in the future of $\mathcal{N}$. The data that have to be prescribed are essentially the so-called null data on $\mathcal{N}$ and $\mathscr{I}$, i.e. those parts of the rescaled Weyl tensor that are entirely intrinsic to the respective null hypersurfaces. In the case of $\mathscr{I}$, the null datum is exactly the radiation field.

Theorem 3 (Kánnár [104]): For given smooth null data on an ingoing null hypersurface $\mathcal{N}$ and a smooth radiation field on the part $\mathcal{I}$ of $\mathscr{I}^{-}$that is to the future of the intersection $S$ of $\mathcal{N}$ with $\mathscr{I}^{-}$and certain data on $S$, there exists a smooth solution of Einstein's vacuum equations in the future of $\mathcal{N} \cup \mathcal{I}$ that implies the given data on $\mathcal{N} \cup \mathcal{I}$.

The result is in complete agreement with Sachs' earlier analysis of the asymptotic characteristic initial value problem based on formal expansion methods [145].

Another case is concerned with the existence of solutions representing pure radiation. These are vacuum solutions characterized by the fact that they are smoothly extensible through past time-like infinity, i.e. by the regularity of the point $i^{-}$. This case has been treated in [60, 62]. A solution of this kind is uniquely characterized by its radiation field, i.e. the intrinsic components of the rescaled Weyl tensor on $\mathscr{I}^{-}$. In the analytic case, a formal expansion of the solution at $i^{-}$ can be derived, and growth conditions on the coefficients can be given to ensure convergence of the formal expansion near $i^{-}$. Furthermore, there exists a surprising relation between this type of solutions and static solutions, summarized in

Theorem 4 (Friedrich): With each asymptotically flat static solution of Einstein's vacuum field equations can be associated another solution of these equations that has a smooth conformal boundary $\mathscr{I}^{-}$and for which the point $i^{-}$is regular.

This result establishes the existence of a large class of purely radiative solutions.

Living Reviews in Relativity

http: //www . livingreviews . org/lrr-2004-1/ 
For applications, however, the most important type of initial value problem so far, in the sense that the asymptotic behaviour can be controlled, has been the hyperboloidal initial value problem where data are prescribed on a hyperboloidal hypersurface. This is a space-like hypersurface whose induced physical metric behaves asymptotically like a surface of constant negative curvature (see Section 2.4). In the conformal picture, a hyperboloidal hypersurface is characterized simply by the geometric fact that it intersects $\mathscr{I}$ transversely in a two-dimensional space-like surface. Prototypes of such hypersurfaces are the space-like hyperboloids in Minkowski space-time. In the Minkowski picture they can be seen to become asymptotic to null cones, which suggests that they reach null-infinity. However, the picture is deceiving: The conformal structure is such that the hyperboloids always remain space-like, the null-cones and the hyperboloids never become tangent. The intersection is a two-dimensional surface $S$, a "cut" of $\mathscr{I}$. The data implied by the conformal fields on such a hypersurface are called hyperboloidal initial data. The first result obtained for the hyperboloidal initial value problem states that if the space-time admits a hypersurface that extends smoothly across $\mathscr{I}^{+}$with certain smooth data given on it, then the smoothness of $\mathscr{I}^{+}$ will be guaranteed at least for some time into the future. This is contained in

Theorem 5 (Friedrich [58]): Smooth hyperboloidal initial data on a hyperboloidal hypersurface $\Sigma$ determine a unique solution of Einstein's vacuum field equations that admits a smooth conformal boundary at null-infinity in the future of $\Sigma$.

There exists also a stability result that states that there are solutions that behave exactly like Minkowski space near future time-like infinity:

Theorem 6 (Friedrich [61]): If the hyperboloidal initial data are in a sense sufficiently close to Minkowskian hyperboloidal data, then there exists a conformal extension of the corresponding solution which contains a point $i^{+}$such that $\mathscr{I}^{+}$is the past null cone of that point.

It should be emphasized that this result implies that the physical metric of the corresponding solution is regular for all future times. Thus, the theorem constitutes a (semi-)global existence result for the Einstein vacuum equations.

\subsection{Hyperboloidal initial data}

Now the obvious problem is to determine hyperboloidal initial data. That such data exist follows already from Theorem 4 because one can construct hyperboloidal hypersurfaces together with data on them in any of the radiative solutions whose existence is guaranteed by that theorem. However, one can also construct such data sets in a similar way to the construction of Cauchy data on an asymptotically Euclidean hypersurface by solving the constraint equations implied on the Cauchy surface. Let $\tilde{\Sigma}$ be a hyperboloidal hypersurface that extends out to $\mathscr{I}$ in an asymptotically flat vacuum space-time, touching $\mathscr{I}$ in a two-surface $\partial \Sigma$ that is topologically a two-sphere. The assumptions on $\tilde{\Sigma}$ are equivalent to the fact that $\tilde{\Sigma}:=\Sigma \cup S$ is a smooth Riemannian manifold with boundary, which carries a smooth metric $h_{a b}$ and a smooth function $\Omega$, obtained by restriction of the unphysical metric and the conformal factor. The conformal factor is a defining function for the boundary $\partial \Sigma$ (i.e. it vanishes only on $\partial \Sigma$ with non-vanishing gradient), and together with the metric it satisfies

$$
\tilde{h}_{a b}=\Omega^{-2} h_{a b}
$$

on $\tilde{\Sigma}$, where $\tilde{h}_{a b}$ is the metric induced on $\tilde{\Sigma}$ by the physical metric. Furthermore, let $\tilde{\chi}_{a b}$ be the extrinsic curvature of $\tilde{\Sigma}$ in the physical space-time. Together with $\tilde{h}_{a b}$ it satisfies the vacuum constraint equations,

$$
\tilde{R}-\tilde{\chi}_{a b} \tilde{\chi}^{a b}+\left(\tilde{\chi}^{a}{ }_{a}\right)^{2}=0,
$$




$$
\tilde{\partial}_{a} \tilde{\chi}_{b}^{a}-\tilde{\partial}_{b} \tilde{\chi}_{a}^{a}=0,
$$

where $\tilde{\partial}_{a}$ is the Levi-Civita connection of $\tilde{h}_{a b}$ and $\tilde{R}$ is its scalar curvature.

Several results of increasing generality have been obtained. We discuss only the simplest case here, referring to the literature for the more general results. Assume that the extrinsic curvature is a pure trace term,

$$
\tilde{\chi}_{a b}=\frac{1}{3} c \tilde{h}_{a b}
$$

The momentum constraint (32) implies that $c$ is constant while the hyperboloidal character of $\tilde{\Sigma}$ implies that $c \neq 0$. With these simplifications and a rescaling of $\tilde{h}_{a b}$ with a constant factor, the Hamiltonian constraint (31) takes the form

$$
\tilde{R}=-6 .
$$

A further consequence of the condition (30) is the vanishing of the magnetic part $B_{a b}$ of the Weyl tensor. For any defining function $\omega$ of the boundary, the conformal factor has the form $\Omega=u^{-2} \omega$. Expressing Equation (33) in terms of the unphysical quantities $h_{a b}$ and $\Omega$ yields the single secondorder equation

$$
8 \omega^{2} \Delta u-8 \omega \partial^{a} \omega \partial_{a} u+\left(\omega^{2} R-4 \omega \Delta \omega+6 \partial^{a} \omega \partial_{a} \omega\right) u=-6 u^{5} .
$$

This equation is a special case of the Lichnérowicz equation and is sometimes also referred to as the Yamabe equation. For a given metric $h_{a b}$ and boundary defining function $\omega$, it is a second-order, non-linear equation for the function $u$. Note that the principal part of the equation degenerates on the boundary. Therefore, on the boundary, the Yamabe equation degenerates to the relation

$$
u \partial^{a} \omega \partial_{a} \omega=u^{5} \text {. }
$$

Note also that $u=0$ is a solution of Equation (34) which, however, is not useful for our purposes because it does not give rise to a meaningful conformal factor $\Omega$. Therefore, we require that $u$ be strictly positive and bounded on the boundary. Then the relation above determines the boundary values of $u$ in terms of the function $\omega$. Taking derivatives of Equation (34), one finds that also the normal derivative of $u$ is fixed on the boundary in terms of the second derivative of $\omega$.

A given metric $\tilde{h}_{a b}$ does not fix a unique pair $\left(h_{a b}, \Omega\right)$. Therefore, Equation (34) has the property that, for fixed $\omega$, rescaling the metric $h_{a b}$ with an arbitrary smooth non-vanishing function $\theta$ on $\Sigma \cup S$ according to $h_{a b} \mapsto \theta^{-4} h_{a b}$ results in a rescaling of the solution $u$ of Equation (34) according to $u \mapsto \theta u$ and, hence, a change in the conformal factor $\Omega \mapsto \theta^{-2} \Omega$.

Now we define the trace-free part $s_{a b}=\phi_{a b}-\frac{1}{3} h_{a b} \phi_{c}{ }^{c}$ of the projection $\phi_{a b}$ of the trace-free part of the unphysical Ricci tensor onto $\Sigma$, and consider the equations

$$
\begin{aligned}
& s_{a b}=-\Omega^{-1}\left(\partial_{a} \partial_{b} \Omega-\frac{1}{3} h_{a b} \Delta \Omega\right), \\
& E_{a b}=-\Omega^{-1}\left(R_{a b}-\frac{1}{3} h_{a b} R-s_{a b}\right),
\end{aligned}
$$

which follow from the Equations (20) and (16), respectively. Together with the fields $h_{a b}, \Omega$, $s=\frac{1}{3} \Delta \Omega$, they provide initial data for all the quantities appearing in the evolution equations under the given assumptions. As they stand, these expressions are formally singular at the boundary and one needs to worry about the possibility of a smooth extension of the field to $\partial \Sigma$. This question was answered in [5], where the following theorem was proved:

Theorem 7: Suppose $(\Sigma, h)$ is a three-dimensional, orientable, compact, smooth Riemannian manifold with boundary $\partial \Sigma$. Then there exists a unique solution $u$ of Equation (34), and the following conditions are equivalent:

Living Reviews in Relativity

http: //www. livingreviews.org/Irr-2004-1/ 
(1) The function $u$ as well as the tensor fields $s_{a b}$ and $E_{a b}$ determined on the interior $\tilde{\Sigma}$ from $h$ and $\Omega=u^{-2} \omega$ extend smoothly to all of $\Sigma$.

(2) The conformal Weyl tensor computed from the data vanishes on $\partial \Sigma$.

(3) The conformal class of $h$ is such that the extrinsic curvature of $\partial \Sigma$ with respect to its embedding in $(\Sigma, h)$ is pure trace.

Condition (3) in Theorem 7 is a weak restriction on the conformal class of the metric $h$ on $\Sigma$, since it is only effective on the boundary. It is equivalent to the fact that in the space-time that evolves from the hyperboloidal data, null-infinity $\mathscr{I}$ is shear-free. Interestingly, the theorem only requires $\Sigma$ to be orientable and does not restrict the topology of $\Sigma$ any further.

This theorem gives the answer in a highly simplified case because the freedom in the extrinsic curvature has been suppressed. But there are also several other, less restrictive, treatments in the literature. In [3, 4] the assumption (30) is dropped, allowing for an extrinsic curvature that is almost general apart from the fact that the mean curvature is required to be constant. In [101] also this requirement is dropped (but, in contrast to the other works, there is no discussion of smoothness of the implied conformal initial data), and in [103] the existence of hyperboloidal initial data is discussed for situations with a non-vanishing cosmological constant.

The theorem states that one can construct the essential initial data for the evolution once Equation (34) has been solved. The data are given by expressions that are formally singular at the boundary because of the division by the conformal factor $\Omega$. This is of no consequences for the analytical considerations if Condition (3) in Theorem 7 is satisfied. However, even then it is a problem for the numerical treatments because one has to perform a limit process to get to the values of the fields on the boundary. This is numerically difficult. Therefore, it would be desirable to solve the conformal constraints directly. It is clear from Equations $(82,83,84,85,86$, $87,88,89,90,91,92)$ that the conformal constraints are regular as well. Some of the equations are rather simple but the overall dependencies and interrelations among the equations are very complicated. At the moment there exists no clear analytical method (or even strategy) for solving this system. An interesting feature appears in connection with Condition (3) in Theorem 7 and analogous conditions in the more general cases. The necessity of having to impose this condition seems to indicate that the development of hyperboloidal data is not smooth but in general at most $\mathcal{C}^{2}$. If the condition were not imposed then logarithms appear in an expansion of the solution of the Yamabe equation near the boundary, and it is rather likely that these logarithmic terms will be carried along with the time evolution, so that the developing null-infinity loses differentiability. Thus, the conformal boundary is not smooth enough and, consequently, the Weyl tensor need not vanish on $\mathscr{I}$ which, in addition, is not necessarily shear-free. The Sachs peeling property is not completely realized in these situations. One can show [3] that generically hyperboloidal data fall into the class of "poly-homogeneous" functions, which are (roughly) characterized by the fact that they allow for asymptotic expansions including logarithmic terms. This behaviour is in accordance with other work [166] on the smoothness of $\mathscr{I}$, in particular with the Bondi-Sachs type expansions, which were restricted by the condition of analyticity (i.e. no appearance of logarithmic terms). It is also consistent with the work of Christodoulou and Klainerman.

Solutions of the hyperboloidal initial value problem provide pieces of space-times that are semiglobal in the sense that their future or past developments are determined depending on whether the hyperboloidal hypersurface intersects $\mathscr{I}^{+}$or $\mathscr{I}^{-}$. However, the domain of dependence of a hyperboloidal initial surface does not include space-like infinity and one may wonder whether this fact is the reason for the apparent generic non-smoothness of null-infinity. Is it not conceivable that the possibility of making a connection between $\mathscr{I}^{+}$and $\mathscr{I}^{-}$across $i^{0}$ to build up a global space-time automatically excludes the non-smooth data? If we let the hyperboloidal initial surface approach space-like infinity, it might well be that Condition (3) in Theorem 7 imposes additional 
conditions on asymptotically flat Cauchy data at spatial infinity. These conditions would make sure that the development of such Cauchy data is an asymptotically flat space-time, in particular that it has a smooth conformal extension at null-infinity.

These questions give some indications about the importance of gaining a detailed understanding of the structure of gravitational fields near space-like infinity. One of the difficulties in obtaining more information about the structure at space-like infinity is the lack of examples that are general enough. There exist exact radiative solutions with boost-rotation symmetry [22]. They possess a part of a smooth null-infinity, which, however, is incomplete. This is a general problem because the existence of a complete null-infinity with non-vanishing radiation restricts the possible isometry group of a space-time to be at most one-dimensional with space-like orbits [14]. Some of the boostrotation symmetric space-times even have a regular $i^{0}$; thus they have a vanishing ADM mass. Other examples exist of space-times that are solutions of the Einstein-Maxwell [39] or EinsteinYang-Mills [15] equations. They have smooth and complete null-infinities. However, they were constructed in a way that enforces the field to coincide with the Schwarzschild or the ReissnerNordström solutions near $i^{0}$. So they are not general enough to draw any conclusions about the generic behaviour of asymptotically flat space-times near $i^{0}$.

Recently, the still outstanding answer to the question as to whether there exist at all global asymptotically simple vacuum space-times with smooth null-infinity could be answered in the affirmative. Corvino [35] has shown that there exist asymptotically flat metrics on $\mathbb{R}^{3}$ with vanishing scalar curvature that are spherically symmetric outside a compact set. His method of construction consists of a gluing procedure by which a scalar flat and asymptotically flat metric can be deformed in an annular region in such a way that it can be glued with a predetermined degree of smoothness to Schwarzschild data while remaining scalar flat. Very recently a generalisation of this construction to non-time-symmetric initial data has been presented by Corvino and Schoen [36].

Such a metric satisfies the constraint equations implied by the vacuum Einstein equations on a space-like hypersurface of time symmetry. It evolves into a space-time that is identical to the Schwarzschild solution near space-like infinity. Within this space-time, which exists for some finite time interval, one can now find hyperboloidal hypersurfaces on which hyperboloidal initial data are induced. This hypersurface can be chosen close enough to the initial Cauchy surface so that it intersects the domain of dependence of the asymptotic region of the initial hypersurface where the data are Schwarzschild. This means that the data implied on the hyperboloidal hypersurface are also Schwarzschild close to $\mathscr{I}$ so that the conditions of Theorem 7 are clearly satisfied.

If one could now apply Friedrich's stability result (Theorem 6), then the existence of global asymptotically flat space-times with smooth extension to null-infinity would have been established. However, one assumption in this theorem is that the data be "sufficiently small". Therefore, one needs to check that one can in fact perform Corvino's construction in the limit of vanishing ADM mass while maintaining a sufficiently "large" Schwarzschild region around $i^{0}$ so that one can find enough hyperboloidal hypersurfaces intersecting the Schwarzschild region in the time development of the data. Chruściel and Delay [32] (see also the erratum in [33]) have adapted Corvino's construction to that situation, and show that it is possible to construct time-symmetric initial data that are Schwarzschild outside a fixed compact region and with a fixed degree of differentiability in the limit $m_{\mathrm{ADM}} \rightarrow 0$. From these data one can construct hyperboloidal hypersurfaces of the type required in Theorem 6 on which hyperboloidal data are induced which satisfy the smallness criterion of that theorem. Hence, there exists a complete space-time in the future of the initial Cauchy surface that admits a conformal extension to null-infinity as smooth as one wishes. Since the argument can also be applied towards the past, one has shown the existence of global spacetimes with smooth $\mathscr{I}$.

Living Reviews in Relativity

http://www . livingreviews . org//rr-2004-1/ 


\subsection{Space-like infinity}

As indicated above, the problem at $i^{0}$ is one of the most urgent and important ones related to the conformal properties of isolated systems. However, it is also one of the most complicated, and a thorough discussion of all its aspects is not possible here. We will try to explain in rough terms what the new developments are, but we have to refer to [68] for the rigorous statements and all the details.

There have been several approaches over the years towards a treatment of space-like infinity. Geroch [79] gave a geometric characterization along the same lines as for null-infinity based on the conformal structure of Cauchy surfaces. He used his construction to define multipole moments for static space-times [76, 77], later to be generalized to stationary space-times by Hansen [87]. It was shown by Beig and Simon [20, 153] that the multipole moments uniquely determine a stationary space-time and vice versa.

Different geometric characterizations of spatial infinity in terms of the four-dimensional geometry were given by Sommers [154], Ashtekar, and Hansen [11, 7], and by Ashtekar and Romano [12]. The difficulties in all approaches that try to characterize the structure of gravitational fields at space-like infinity in terms of the four-dimensional geometry arise from the lack of general results about the evolution of data near spatial infinity. Since there are no radiating solutions that are general enough at spatial infinity to provide hints, one is limited more or less to one's intuition. So all these constructions essentially impose "reasonable" asymptotic conditions on the gravitational field at $i^{0}$, and from them derive certain nice properties of space-times which satisfy these conditions. But there is no guarantee that there are indeed solutions of the Einstein equations that exhibit the claimed asymptotic behaviour. In a sense, all these characterisations are implicit definitions of certain classes of space-times (namely those that satisfy the imposed asymptotic conditions). What is needed is an analysis at space-like infinity which is not only guided by the geometry but which also takes the field equations into account (see e.g. [18, 19] for such attempts using formal power series).

Recently, Friedrich [68] has given such an analysis of space-like infinity, which is based exclusively on the initial data, the field equations and the conformal structure of the space-time. In this representation several new aspects come together. First, in order to simplify the analysis, an assumption on the initial data (metric and extrinsic curvature) on an asymptotically Euclidean hypersurface $\tilde{\Sigma}$ is made. Since the focus is on the behaviour of the fields near space-like infinity, the topology of $\tilde{\Sigma}$ is taken to be $\mathbb{R}^{3}$. It is assumed that the data are time-symmetric $\left(\tilde{\chi}_{a b}=0\right)$ and that on $\tilde{\Sigma}$ a (negative definite) metric $\tilde{h}_{a b}$ with vanishing scalar curvature is given. Let $\Sigma$ be the conformal completion of $\left(\tilde{\Sigma}, \tilde{h}_{a b}\right)$ which is topologically $S^{3}$, obtained by attaching a point $i$ to $\tilde{\Sigma}$, and assume furthermore that there exists a smooth positive function $\Omega$ on $\Sigma$ with $\Omega(i)=0$, $d \Omega(i)=0$, and Hess $\Omega(i)$ negative definite. Furthermore, the metric $h_{a b}=\Omega^{2} \tilde{h}_{a b}$ extends to a smooth metric on $S$. Thus, the three-dimensional conformal structure defined by $\tilde{h}_{a b}$ is required to be smoothly extensible to the point $i$.

From these assumptions follows that the conformal factor near $i$ is determined by two smooth functions $U$ and $W$, where $U$ is characterized by the geometry near $i$, while $W$ collects global information because $W(i)=\frac{1}{2} m_{\mathrm{ADM}}$, while $U(i)=1$. With this information the rescaled Weyl tensor, the most important piece of initial data for the conformal field equations, near $i$ is found to consist of two parts, a "massive" and a "mass-less" part. Under suitable conditions, the mass-less part, determined entirely by the local geometry near $i$, can be extended in a regular way to $i$, while the massive part always diverges at $i$ as $1 /|x|^{3}$ in a normal coordinate system $\left(x^{1}, x^{2}, x^{3}\right)$ at $i$ unless the ADM mass vanishes.

In order to analyze the singular behaviour of the initial data in more detail, the point $i$ is blown up to a spherical set $I^{0}$ essentially by replacing it with the sphere of unit vectors at $i$. Roughly speaking, this process yields a covering space $\mathcal{C}$ of (a suitable neighbourhood of $i$ in) $\Sigma$ projecting 
down to $\Sigma$ which has the following properties: The pre-image $I^{0}$ of $i$ is an entire sphere while any other point on $\Sigma$ has exactly two pre-image points. There exists a coordinate $r$ on $\mathcal{C}$ which vanishes on $I^{0}$, and which is such that on each pair of pre-image points it takes values $r$ and $-r$, respectively. The actual blow-up procedure involves a rather complicated bundle construction that also takes into account the tensorial (respectively spinorial) nature of the quantities in question. The reader is referred to [68] for details.

Consider now a four-dimensional neighbourhood of space-like infinity. The next important step is the realization that, in order to take full advantage of the conformal structure of space-time, it is not enough to simply allow for metrics that are conformally equivalent to the physical metric, but that one should also allow for more general connections. Instead of using a connection that is compatible with a metric in the conformal class, one may use a connection $\nabla_{a}$ compatible with the conformal structure, i.e. that satisfies the condition

$$
\nabla_{c} \tilde{g}_{a b}=2 \lambda_{c} \tilde{g}_{a b}
$$

for some one-form $\lambda_{a}$. If $\lambda_{a}$ is exact, then one can find a metric in the conformal class for which $\nabla_{a}$ is the Levi-Civita connection. Generally, however, this will not be the case. This generalization is motivated by the use of conformal geodesics as indicated below, and its effect is to free up the conformal factor, which we call $\Theta$ to distinguish it from the conformal factor $\Omega$ given on the initial surface $\Sigma$, from the connection. (Recall that two connections, that are compatible with metrics in the same conformal class, differ only by terms which are linear in the first derivative of the conformal factor relating the metrics.) As a consequence, the conformal field equations, when expressed in terms of a generalized connection, do not any longer contain an equation for the conformal factor. It appears, instead, as a gauge source function for the choice of conformal metric. Additionally, a free one-form appears which characterizes the freedom in the choice of the conformal connection.

To fix this freedom, Friedrich uses conformal geodesics [73]. These curves generalize the concept of auto-parallel curves. They are given in terms of a system of ordinary differential equations (ODE's) for their tangent vector together with a one-form along them. In coordinates this corresponds to a third-order ODE for the parameterization of the curve. Their crucial property is that they are defined entirely by the four-dimensional conformal structure with no relation to any specific metric in that conformal structure.

A time-like congruence of such curves is used to set up a "Gauß" coordinate system in a neighbourhood of $i^{0}$ and to define a conformal frame, a set of four vector fields that are orthonormal for some metric in the conformal class. This metric in turn defines a conformal factor $\Theta$ that rescales it to the physical metric. The one-form determined by the conformal geodesics defines a conformal connection $\nabla_{a}$, thus fixing the freedom in the connection. In this way, the gauge is fixed entirely in terms of the conformal structure. One may wonder whether the choice of conformal "Gauß" coordinates is a practical one, since it is well known that the usual Gauß coordinates are prone to develop singularities very quickly. However, conformal geodesics are in general not geodesics in the physical space-time, and parallel transport with a Weyl connection is not the same as parallel transport in the physical space-time. If one thinks of the usual Gauß coordinates as being constructed from the world-lines of freely falling particles, then one should think of the conformal geodesics as the world-lines of some fiducial "particles" that experience a force determined by the conformal structure of the space-time. This "conformal force" could counteract the attractive nature of the gravitational field under certain conditions, thereby delaying the intersection of the world-lines and the formation of coordinate singularities. In fact, Friedrich [70] has shown that there exist global conformal Gauß coordinates on the Kruskal extension of the Schwarzschild spacetime that extend smoothly beyond null-infinity. Thus, one would hope that the conformal Gauß systems can be used to globally cover more general space-times as well. This would make them an ideal tool for use in numerical simulations because they are intrinsically defined so that the

Living Reviews in Relativity

http://www . livingreviews . org/lrr-2004-1/ 
numerical results would also have an intrinsic meaning. Furthermore, as outlined below, the use of a conformal Gauß system simplifies the field equations considerably.

If the physical space-time is a vacuum solution of the Einstein equations, then one can say more about the behaviour of the conformal factor $\Theta$ along the conformal geodesics: It is a quadratic function of the natural parameter $\tau$ along the curves, vanishing at exactly two points, if the initial conditions for the curves are chosen appropriately. The vanishing of $\Theta$ indicates the intersection of the curves with $\mathscr{I}^{ \pm}$. The intersection points are separated by a finite distance in the parameter $\tau$.

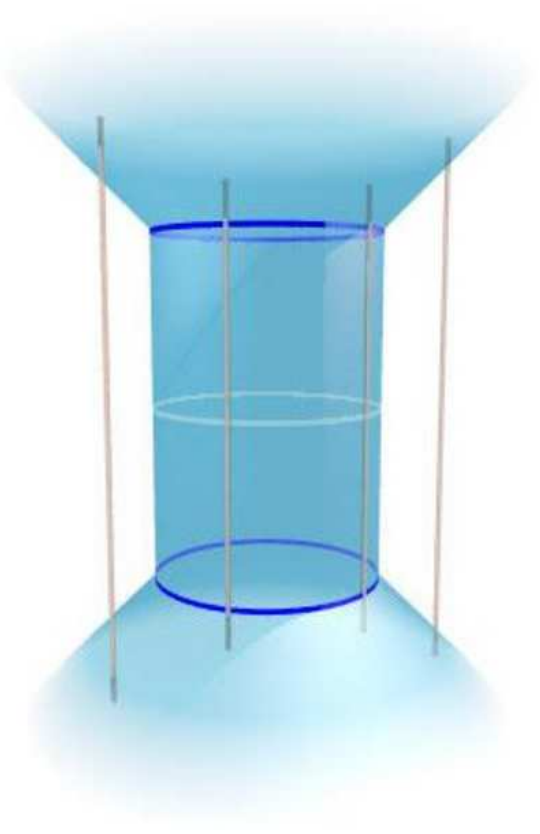

Figure 8: The geometry near space-like infinity: The "point" $i^{0}$ has been blown up to a cylinder that is attached to $\mathscr{I}^{-}$and $\mathscr{I}^{+}$. The physical space-time is the exterior part of this "stovepipe". The "spheres" $I^{ \pm}$and $I^{0}$ are shown in blue and light green, respectively. The brown struts symbolize the conformal geodesics used to set up the construction. Note that they intersect $\mathscr{I}$ and continue into the unphysical part.

Now, one fixes an initial surface $\Sigma$ with data as described above, and the conformal geodesics are used to set up the coordinate system and the gauge as above. When the blow-up procedure is performed for $\Sigma$, a new finite representation of space-like infinity is obtained, which is sketched in Figure 8.

The point $i$ on the initial surface has been replaced by a sphere $I^{0}$, which is carried along the conformal geodesics to form a finite cylinder $I$. The surfaces $\mathscr{I}^{ \pm}$are the surfaces on which the conformal factor $\Theta$ vanishes. They touch the cylinder in the two spheres $I^{ \pm}$, respectively. The conformal factor $\Theta$ vanishes with non-vanishing gradient on $I$ and on $\mathscr{I}^{ \pm}$, while on the spheres $I^{ \pm}$ its gradient also vanishes.

In this representation there is for the first time a clean separation of the issues that determine the structure of space-like infinity: The spheres $I^{ \pm}$are the places where " $\mathscr{I}$ touches $i^{0}$ " while the finite cylinder $I$ serves two purposes. On the one hand, it represents the endpoints of space-like geodesics approaching from different directions, while, on the other hand, it serves as the link between past and future null-infinity. The part "outside" the cylinder where $r$ is positive between 
the two null surfaces $\mathscr{I}^{ \pm}$corresponds to the physical space-time, while the part with $r$ negative is not causally related to the physical space-time but constitutes a smooth extension. For easy reference, we call this entire space an extended neighbourhood of space-like infinity.

The conformal field equations, when expressed in the conformal Gauß gauge of this generalized conformal framework, yield a system of equations that has properties similar to the earlier version: It is a system of equations for a frame, the connection coefficients with respect to the frame, and the curvature, split up into the Ricci and the Weyl parts; they allow the extraction of a reduced system that is symmetric hyperbolic and propagates the constraint equations. Its solutions yield solutions of the vacuum Einstein equations whenever $\Theta>0$. The Bianchi identities, which form the only sub-system consisting of partial differential equations, again play a key role in the system. Due to the use of the conformal Gauß gauge, all other equations are simply transport equations along the conformal geodesics.

The reduced system is written in symbolic form as

$$
\mathbf{A}^{\tau} \frac{\partial}{\partial \tau} \mathbf{u}+\mathbf{A}^{r} \frac{\partial}{\partial r} \mathbf{u}+\mathbf{A}^{\theta} \frac{\partial}{\partial \theta} \mathbf{u}+\mathbf{A}^{\phi} \frac{\partial}{\partial \phi} \mathbf{u}=\mathbf{B u}
$$

with symmetric matrices $\mathbf{A}^{\tau}, \mathbf{A}^{r}, \mathbf{A}^{\theta}, \mathbf{A}^{\phi}$, and $\mathbf{B}$ which depend on the unknown $\mathbf{u}$ and the coordinates $(\tau, r, \theta, \phi)$. This system as well as initial data for it, first defined only on the original space-time, can be extended in a regular way to an extended neighbourhood of space-like infinity, which allows for the setup of a regular initial value problem at space-like infinity. Its properties are most interesting: When restricted to $I^{0}$, the initial data coincide necessarily with Minkowski data, which together with the vanishing of $\Theta$ implies that on the entire cylinder $I$ the coefficient matrix $\mathbf{A}^{r}$ vanishes. Thus, the system (37) degenerates into an interior symmetric hyperbolic system on $I$. Therefore, the finite cylinder $I$ is a total characteristic of the system. The two null-infinities $\mathscr{I}^{ \pm}$ are also characteristics, and at the intersections $I^{ \pm}$between them and $I$, the system degenerates: The coefficient matrix $\mathbf{A}^{\tau}$ which is positive definite on $I$ and $\mathscr{I}^{ \pm}$looses rank on $I^{ \pm}$.

The fact that $I$ is a total characteristic implies that one can determine all fields on $I$ from data given on $I^{0}$. $I$ is not a boundary on which one could specify in- or outgoing fields. This is no surprise, because the system (37) yields an entirely structural transport process that picks up data delivered from $\mathscr{I}^{-}$via $I^{-}$, and moves them to $\mathscr{I}^{+}$via $I^{+}$. It is also consistent with the standard Cauchy problem where it is known that one cannot specify any data "at infinity".

The degeneracy of the equations at $I^{ \pm}$means that one has to take special precautions to make sure that the transitions from and to $\mathscr{I}^{ \pm}$are smooth. In fact, not all data "fit through the pipe"; Friedrich has derived restrictions on the initial data of solutions of the finite initial value problem that are necessary for regularity through $I^{ \pm}$. These are necessary conditions on the conformal class of the initial data, stating that the Cotton tensor and all its symmetrized and trace-removed derivatives should vanish at the point $i$ in the initial surface. If this is not the case, then the solution of the intrinsic system will develop logarithmic singularities, which will then probably spread across null-infinity, destroying its smoothness. So here is another concrete indication that initial data have to be restricted, albeit in a rather mild way, in order for the smooth picture of asymptotic flatness to remain intact.

In order to get more insight into the character of the transport equations along the cylinder $I$ and, in particular, to see whether these conditions are also sufficient, one can take advantage of the group theoretical origin of the blow-up procedure, and write all the relevant quantities according to their tensorial character as linear combinations of a complete set of functions on the sphere. (According to the Peter-Weyl theorem, the matrix entries of irreducible representations of $S U(2)$ form a complete system of functions on $S U(2)$, these functions being closely related to the spin-spherical harmonics [134].) Insertion into the transport equations and restriction to the cylinder $I$ yields a set of ODE's for the coefficients along the conformal geodesics that generate $I$. Taking successive $r$-derivatives and restricting to the cylinder yields a hierarchy of such ODE's.

Living Reviews in Relativity

http://www . livingreviews . org//rr-2004-1/ 
The system of ODE's obtained at order $n$ can be solved provided a solution up to order $n-1$ is known. Thus, given initial conditions, one can determine recursively the expansion coefficients and thereby obtain a Taylor approximation of the fields in a neighbourhood of $I$. These calculations are rather cumbersome and can be performed only with the aid of computer algebra.

In view of the unknown status of the above condition on the Cotton tensor, it is clear that a particularly interesting scenario is obtained for conformally flat initial data. For such data, the Cotton tensor vanishes identically, so that the conditions above are satisfied. If any logarithms appear in the solutions of the hierarchy of ODE's, then this implies that the vanishing of the Cotton tensor and its symmetrized, trace-removed derivatives is only necessary and not sufficient.

In [160] Valiente Kroon has carried out these calculations up to order $n=6$ in the general case and up to order $n=8$ for data that were in addition assumed to be axi-symmetric. The rather surprising result is this: Up to order $n=4$ the solutions do not contain any logarithms. This changes with $n=5$, where there are logarithmic terms that contribute additively to the solution with coefficients $G^{(5)}$ proportional to the Newman-Penrose constants. This means that the solution is smooth up to $n=5$ only if the Newman-Penrose constants vanish. Assuming this, then for $n=6$ the solutions will again have an additive logarithmic term with some coefficients $G^{(6)}$ (higher-order Newman-Penrose "constants") whose vanishing is necessary for smoothness of the solution. This picture returns at the orders $n=7$ and $n=8$, where again the vanishing of certain quantities $G^{(n)}$ is necessary for the solution to be free of logarithms.

From the explicit algebraic expressions for the $G^{(n)}$ up to order 8 , one can guess a formula that expresses them for arbitrary orders in terms of certain expansion coefficients of the conformal factor on the initial hypersurface. Then, the vanishing of the $G^{(n)}$ implies that the expansion coefficients of the conformal factor are correlated in a very specific way. In the special case of the Schwarzschild space-time (which is spherically symmetric, hence has conformally flat initial data), one can compute the expansion coefficients of the conformal factor explicitly, and one finds that they are correlated in exactly the way implied by the vanishing of the $G^{(n)}$. This observation seems to indicate that the time evolution of an asymptotically Euclidean, conformally flat, and smooth initial data set admits a smooth extension to null-infinity near space-like infinity if and only if it agrees with the Schwarzschild solution (the only static solution in the conformal class of the data) to all orders at the point $i$. This conjecture seems to imply that the developments of the Brill-Lindquist and Misner initial data sets have non-smooth null-infinities.

While the conditions on the Cotton tensor are entirely local to $i$, this is not so for the additional conditions discussed above. They involve the part $W$ of the conformal factor which is related to the ADM mass of the system and which is obtained by solving an elliptic equation. This part is therefore determined by properties of the initial data that are not local to $i$. To what extent it is really the global structure of the initial data which is involved here is not so clear since the results by Corvino [35] and Corvino-Schoen [36] show that one can deform initial data in certain annular regions of the initial hypersurface while keeping the asymptotics and the inner regions untouched.

These observations also shine a new light on the static, respectively stationary, solutions. It might well be that they play a fundamental role in the construction of asymptotically flat spacetimes. If it turns out that such space-times have to be necessarily static (or stationary in the nontime-symmetric case) near $i^{0}$ in order to have a smooth conformal extension, then this implies that there are no gravitational waves allowed in the neighbourhood of space-like infinity. In particular, this means that incoming radiation has to die off in the infinite future, while the system cannot have emitted gravitational waves for all times in the infinite past. Whether this is a reasonable scenario for an isolated system is a question of physics. It has to be answered by investigating whether it is possible to discuss realistic physical processes like radiation emission and scattering in a meaningful way such as whether one can uniquely define physical quantities like energy-momentum, angular momentum, radiation field, etc.

This touches upon the question as to how physically relevant the assumption of a smooth 
asymptotic structure will in the end turn out to be. It is after all an idealisation, which we use as a tool to describe an isolated system. If we want to find out whether this idealisation captures physically relevant scenarios, we first have to know the options, i.e. we need to have detailed knowledge about the mathematical situations that can arise. Whether these situations are general enough to admit all physical situations that one would consider as reasonable remains an open question.

The setting described in the above paragraphs certainly provides the means to analyze the consequences of the conformal Einstein evolution near space-like infinity and to understand the properties of gravitational fields in that region. The finite picture allows the discussion of the relation between various concepts that are defined independently at null and space-like infinity. As one application of this kind, Friedrich and Kánnár [71] have related the Newman-Penrose constants, which are defined by a surface integral over a cut of $\mathscr{I}^{+}$, to initial data on $\Sigma$. The cut of $\mathscr{I}^{+}$is pushed down towards $I^{+}$, where it is picked up by the transport equations of system (37). In a similar way, one can relate the Bondi and ADM masses of a space-time.

\subsection{Going further}

Readers who are interested in obtaining information about the current status of existence theorems in general relativity are referred to Rendall's Living Reviews article [138].

Although we have restricted ourselves to the case of a vanishing cosmological constant this does not mean that other cases have not been analyzed. Friedrich [63] has shown the existence of asymptotically simple de Sitter type solutions of the Einstein-Maxwell-Yang-Mills equations and stability of de Sitter space. More recently [65], with the general version of his conformal field equations, he was able to demonstrate the existence of asymptotically simple anti-de Sitter type solutions.

It should also be mentioned that some work has gone into generalizing the Bondi-Sachs calculations to poly-homogeneous space-times [34, 161, 162].

The structure of space-like infinity has been discussed here only in a rather cursory way. A very recent account of the relationship between static solutions and the role they play can be found in [53]. Readers who are interested in a more detailed treatment of the subject in general are referred to a recent review by Friedrich [69].

Living Reviews in Relativity

http: //www. livingreviews . org/lrr-2004-1/ 


\section{Numerical Issues}

In this section we discuss some of the issues that have to be confronted in a numerical implementation of the Einstein equations and, in particular, of the conformal field equations to solve the hyperboloidal initial value problem. The material presented in this section is based to a large extent on work by Hübner [89, 90, 91, 92, 94, 95] and Frauendiener [45, 46, 47, 48, 49]. These are also the references which should be consulted for a more detailed discussion of the various numerical schemes. The presentation here will be rather cursory in order to keep it reasonably short.

\subsection{Why use the conformal method for numerical relativity?}

At the moment, three major approaches towards the numerical treatment of asymptotically flat space-times exist. They are based on three different formulations of the Einstein equations:

- the standard Cauchy problem using the Einstein equations in either ADM-like form or in one of the current hyperbolic formulations,

- the characteristic initial value problem for the standard Einstein equations in Bondi- or Newman-Penrose form, and

- the Cauchy problem for the conformal field equations.



Figure 9: The geometry of the standard Cauchy approach. The green lines are surfaces of constant time. The blue line indicates the outer boundary.

Let us discuss the main properties of each of these approaches with respect to their treatment of infinity. In this connection it is useful to look at conformal diagrams. In all three cases the 
physical situation is the same. A source, indicated by a shaded brown region, evolves between the time-like past and future infinities $i^{ \pm}$and thereby emits gravitational radiation, which escapes towards future null infinity $\mathscr{I}^{+}$. The three methods differ in how they introduce an evolution process in order to simulate the physical system. The goal is to obtain the radiation data that are received by an observer on $\mathscr{I}^{+}$as accurately as possible (see [49] for a discussion of the issues involved in the reception process).

In Figure 9 we sketch the geometry implied by solving the Cauchy problem for the standard Einstein equations in any of the various forms that exist today: ADM formulation, old [6] and improved [17], or any one of the hyperbolic formulations such as e.g. in [1, 74]. In this approach the physical space-time is decomposed into space and time by the introduction of a time coordinate. The hypersurfaces of constant physical time are Cauchy surfaces and, hence, asymptotically Euclidean. They are indicated by green lines. The blue line corresponds to a (normally time-like) hypersurface which has to be introduced because for the standard Einstein equations, formulated in the physical space-time, null-infinity is infinitely far away. The numerical grids are distributed along the constant time hypersurfaces, and with each time-step they evolve from one hypersurface to the next. They have outermost grid-points which, in the course of the evolution, move along that time-like hypersurface.

Thus, inevitably, we are facing the task of solving an initial boundary value problem (IBVP) for the Einstein equations. Such problems are notoriously difficult to treat numerically. This, in fact, is one of the big problems in scientific computing as witnessed by the following quotation [159]:

The difficulties caused by boundary conditions in scientific computing would be hard to overemphasize. Boundary conditions can easily make the difference between a successful and an unsuccessful computation, or between a fast and a slow one. Yet in many important cases, there is little agreement about what the proper treatment of the boundary should be.

One of the sources of difficulty is that although some numerical boundary conditions come from discretizing the boundary conditions for the continuous problem, other 'artificial' or 'numerical boundary conditions' do not. The reason is that the number of boundary conditions required by a finite difference formula depends on its stencil, not on the equation being modeled. Thus even a complete mathematical understanding of the initial boundary value problem to be solved (which is often lacking) is in general not enough to ensure a successful choice of numerical boundary conditions. This situation reaches an extreme in the design of what are variously known as 'open', 'radiation', 'absorbing', 'non-reflecting', or 'far-field' boundary conditions, which are numerical artifacts designed to limit a computational domain in a place where the mathematical problem has no boundary at all.

Despite these remarks, perhaps the most basic and useful advice one can offer concerning numerical boundary conditions is this: Before worrying about discretization, make sure to understand the mathematical problem. If the IBVP is ill-posed, no amount of numerical cleverness will lead to a successful computation. This principle may seem too obvious to deserve mention, but it is surprising how often it is forgotten.

While these remarks apply to any numerical problem, they are particularly relevant to numerical relativity.

Until only quite recently no result on the IBVP for the Einstein equations was available. This situation has changed with the work in [72] where it is proved that the IBVP for the Einstein equations in the physical space-time is well-posed for a certain class of boundary conditions. In rough terms, these boundary conditions can be understood as follows: For an arbitrarily specified time-like unit vector tangent to the boundary, we can define a null-tetrad by taking the two real

Living Reviews in Relativity

http: //www . livingreviews . org/Irr-2004-1/ 
null-vectors as lying in the two-dimensional space spanned by that vector and the unit vector normal to the boundary. This fixes the null-tetrad up to boosts in that plane and rotations in the plane orthogonal to it. With respect to that tetrad we can define the (complex) Weyl tensor components $\Psi_{0}$ and $\Psi_{4}$. Then, the boundary conditions have the form

$$
q=-\Psi_{4}+\alpha \Psi_{0}+\beta \bar{\Psi}_{0} .
$$

Here $\alpha, \beta$, and $q$ are complex functions on the boundary. The function $q$ can be freely specified, thus representing the free data, while $\alpha$ and $\beta$ are restricted by some condition, which implies in particular that $|\alpha|^{2}+|\beta|^{2} \leq 1$. They can be regarded as specifying the reflection and transmission properties of the boundary. In the frame defined above, the component $\Psi_{0}$ and $\Psi_{4}$ can be interpreted as the part of the Weyl tensor that propagates orthogonally across the boundary in the outward or inward direction, respectively. The boundary conditions (38) essentially say that we can specify the value of the ingoing part freely and couple some parts of the outgoing field back to it.

Consider the boundary condition with $q=0$ and $\alpha=\beta=0$ viz. $\Psi_{4}=0$. This looks like a completely absorbing boundary condition or, in other words, like the condition of no incoming radiation. However, this is deceiving. Recall that the Weyl tensor components are defined with respect to a time-like unit vector. But there is no distinguished time-like unit-vector available so that they are all equivalent. This means that the boundary conditions, and hence the presence or absence of incoming radiation, are observer dependent. Clearly, the condition of no incoming radiation is not compatible with the Einstein equations anyway, because outgoing waves will always be accompanied by backscattered waves generated by the non-linear nature of the equations. The impossibility of a local completely absorbing boundary condition for the scalar wave equation in flat space has been emphasized already in numerical analysis [43]. The deep reason behind this fact seems to be the Lorentz invariance of the equations. While the conditions in (38) give rise to a well-posed IBVP, they have not found their way into the numerical work yet.

Returning to Figure 9 we see that there are several fundamental difficulties with this (currently standard) approach. Recall that the goal is to obtain radiative information near $\mathscr{I}^{+}$. To achieve this goal we need to arrange for two things: We need to make sure that we simulate an asymptotically flat space-time, and we need to have a stable code in order to get meaningful results. In this approach, both these requirements can only be realized by an appropriate boundary condition. The orange line in the diagram indicates the domain of influence of the boundary. The parts of space-time above that line are influenced by the boundary condition. It is obvious from the causal dependencies that the radiative information that can be accessed using this approach is to a large extent affected by the boundary condition. A bad choice of the boundary condition, in particular one which is not compatible with the Einstein evolution, or one which is not adequate for asymptotic flatness, can ruin any information gained about the radiation.

So we see that a boundary condition has to have several important properties. It must be:

- Physically reasonable: In some way it should express the asymptotic flatness of the space-time manifold.

- Mathematically reasonable: The IBVP should be well-posed with this boundary condition.

- Numerically reasonable: Codes which implement the boundary condition should be stable.

Devising a boundary condition that has these properties has been found to be very hard, if not impossible. There have been several proposals, ranging from specifying Minkowski or Schwarzschild data to matching with linearized solutions of the Einstein equations. Although these conditions are physically reasonable to some extent, they are not compatible with the Einstein evolution and therefore they do not possess the other two properties. Such inappropriate boundary conditions 
can be expected to perform well for small amplitudes and for short times when the non-linear effects can be neglected. However, they will necessarily become unstable in the long run.

We can also see from the diagram that the choice of asymptotically Euclidean hypersurfaces is unfortunate for the following reason. A natural thing to do in order to increase the accuracy of the results is to push the boundary further outward towards infinity. However, pushing along surfaces of constant time brings us closer to $i^{0}$ and not to the interesting parts in the neighbourhood of $\mathscr{I}^{+}$. This implies that the numerical codes not only increase in size but in addition that they have to run longer (in the sense of elapsed physical time) until the interesting regions are covered. The constant time hypersurfaces do not "track the radiation".

One idea [23] to overcome the problems encountered in the standard Cauchy approach is the socalled Cauchy-Characteristic matching procedure [24]. In this approach one introduces a time-like hypersurface, the interface, along which one tries to match a Cauchy problem with a characteristic initial value problem. The Cauchy part provides the interior boundary condition for the characteristic part while the latter provides the outer boundary condition for the former. Thus, the space-time is foliated by hypersurfaces that are partly space-like and partly null, where the causal character changes in a continuous but non-differentiable way across a two-dimensional surface (see Figure 10).

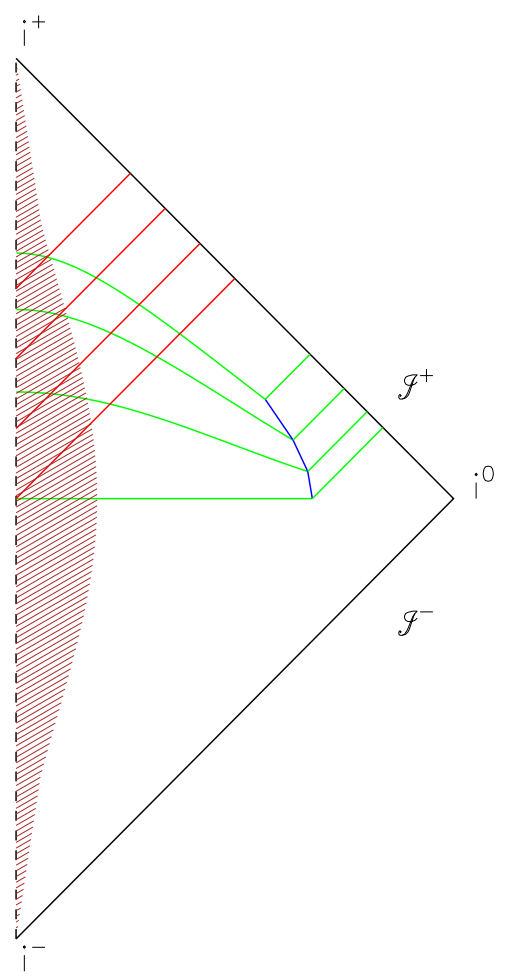

Figure 10: The geometry of Cauchy-Characteristic matching. The blue line indicates the interface between the (inner) Cauchy part and the (outer) characteristic part.

The characteristic part of the hypersurfaces reaches out all the way to $\mathscr{I}^{+}$. The advantage of this procedure is that the problem of finding the correct boundary condition has been eliminated by the introduction of the characteristic part of the scheme.

The characteristic part has been implemented numerically in a very successful way (see the Living Reviews article [164] for a recent review on that topic). It is based on the Bondi-Sachs

Living Reviews in Relativity

http: //www . livingreviews . org/lrr-2004-1/ 
equations for the gravitational field on null-hypersurfaces. Using a "compactified coordinate" along the generators of the null-hypersurfaces it is even possible to "bring null-infinity to finite places". However, this is different from the conformal compactification because the metric is not altered. This necessarily leads to equations that "notice where infinity is" because they degenerate there. Because of this degeneracy the solutions can be singular unless very specific boundary values are prescribed. This is essentially the peeling property of the fields in disguise.



Figure 11: The geometry of the conformal approach. The green lines indicate the foliation of the conformal manifold by hyperboloidal hypersurfaces.

The critical place is, of course, the interface where two completely different codes have to be matched. This is not merely a change in the numerical procedures but the entire setup changes across the interface: the geometry, the independent and dependent variables, and so on. Naturally, this requires a very sophisticated implementation of the interface. Test calculations have been performed successfully for space-times with symmetries (cylindrical, spherical) and/or model equations such as non-linear wave equations. Currently, a combined code for doing the pure gravitational problem in three dimensions is being developed. This is also described in more detail in [164].

Finally, we want to discuss the approach based on the conformal compactification using the conformal field equations (see Figure 11). In this case, the arena is not the physical space-time but some other unphysical manifold, which is conformally related via some conformal factor $\Omega$.

The physical space-time is the part of the conformal manifold on which $\Omega$ is positive. Introducing an appropriate time-coordinate in the conformal manifold leads to a foliation by space-like hypersurfaces that also cover the physical manifold. Those hypersurfaces that intersect $\mathscr{I}^{+}$transversely are hyperboloidal hypersurfaces in the physical space-time. It is important to note that they are submanifolds of the conformal manifold so that they do not stop at $\mathscr{I}^{+}$but continue 
smoothly across $\mathscr{I}$, which is just another submanifold of the conformal manifold, albeit a special one.

Hyperboloidal initial data (see Section 3.4) are given on one such hypersurface and are subsequently evolved with the symmetric hyperbolic (reduced) system of evolution equations. In contrast to the behaviour discussed above in connection with the characteristic evolution, the conformal field equations do not know where $\mathscr{I}$ is. They know that there might be a $\mathscr{I}$ (because the conformal factor is one of the variables in the system) but they have to be told its location on the initial data surface, and from there on this information is carried along by the evolution.

What are the advantages of this kind of formulation? First of all, one might have thought that the question of the correct boundary condition for the IBVP for the Einstein equations has now merely been shifted to the even more complicated problem of finding a boundary condition for the conformal field equations. This is true but irrelevant for the following reason: Suppose we give hyperboloidal initial data on that part of the initial surface that lies inside the physical region. We may now smoothly extend these data into the unphysical region. The structure of the characteristics of the conformal field equations is such that (provided the gauges are chosen appropriately) the outermost characteristic is the light cone. Hence $\mathscr{I}$ is a characteristic for the evolution equations, and this implies that the extension of the initial data into the unphysical region cannot influence the interior. Similarly, it is true that also the conformal field equations need a boundary condition. But since this boundary has been shifted into the unphysical region there is no need for it to be physically reasonable. It is enough to have a boundary condition that is numerically reasonable, i.e. which leads to stable codes. The information generated at the boundary travels at most with the velocity of light and so it cannot swap into the physical region. $\mathscr{I}$ acts as a "one-way membrane". It should be remarked here that this is true for the exact analytical case. Numerically, things are somewhat different but one can expect that any influence from the outside will die away with the order of accuracy of the discretization scheme used (see below).

A further advantage of the conformal approach is the possibility to study global properties of the space-times under consideration. Not only do the hyperboloidal hypersurfaces extend up to and beyond null-infinity, but it is also possible to study the long-time behaviour of gravitational fields. If the initial data are small enough so that future time-like infinity $i^{+}$is a regular point (see Theorem 6), then one can determine in a finite (conformal and computational) time the entire future of the initial surface and therefore a (semi-)global space-time up to (and even beyond) $i^{+}$. This has been successfully demonstrated by Hübner [90] in the case of spherically symmetric calculations and more recently also in higher dimensions.

The calculation of a global space-time up to $i^{+}$has the effect of shrinking the region on the computational domain that corresponds to the physical space-time because $\mathscr{I}^{+}$seems to move through the grid. This means that more and more grid-points are lost for the computation of the physical part. Sometimes it might be more useful to have more resolution there. Then the available gauge freedom can be used to gain complete control over the movement of $\mathscr{I}^{+}$through the grid [46].

The conformal field equations lead to a first order system of approximately sixty equations, depending on the specific formulation and gauge conditions. In the version derived in Appendix 6 there are 65 equations. This is a very large number of equations. However, on the one hand one has to compare this with current proposals for hyperbolic systems for the Einstein equations, which advocate a number of equations of the same order (although there are not even equations for a conformal factor). On the other hand, the variables appearing in the conformal field equations are of a very geometric nature, and for further investigations of the space-times (finding the geodesics, computing forces, etc.), these variables would have to be computed anyway.

But we can be somewhat more specific: The conformal field equations have, roughly, a total number of 60 equations. The conventional codes use roughly 10 equations if they use the standard

Living Reviews in Relativity

http://www. livingreviews.org/lrr-2004-1/ 
Cauchy approach. So there is a factor of 6 between the conformal field equations and the others. But that is not really the point. The complexity (in particular, the memory) scales roughly with the number of gridpoints, and for a 3D code that is $N^{3}$, where $N$ is the number of grid points per dimension. So already doubling $N$ gives a factor of 8 . The upshot of this is that the memory requirements are not dictated by the number of equations, because this is a fixed factor, but by the dimensionality of the code, and this affects both codes in the same way.

A final word concerning the inclusion of matter into the conformal approach: It is clear that this is more complicated than it is for the standard Einstein equations. The reason is that the conformal field equations also contain the (rescaled) Weyl curvature, which couples to the energy-momentum tensor via its derivatives. This means that one needs equations not only for those matter fields that appear in the energy-momentum tensor but also for their derivatives. Furthermore, the fact that under conformal rescalings the trace-free part of the energy-momentum tensor behaves differently compared to its trace, causes additional difficulties. However, these can be overcome under certain circumstances $[63,91]$.

It was mentioned in Section 3.1 that for $\Omega=1$ the conformal field equations reduce to the standard Einstein equations together with the vacuum Bianchi identity. This suggests that one should specify the conformal factor initially to be equal to unity in a region which contains the sources (assuming the sources have spatially compact support) and then decreases until it vanishes on $\mathscr{I}$. Furthermore, the time-derivative of $\Omega$ should be adjusted such that it vanishes in a neighbourhood of the source. In fact, it is possible to do so, but it is not yet known, whether and, if so, for how long one can keep up this condition of constant conformal factor around the source. The proposed procedure does not eliminate the problem that additional equations for the matter variables are needed, but it might reduce the complexity somewhat.

We will now discuss some issues related to the construction of hyperboloidal initial data and the implementation of the evolution equations.

\subsection{Construction of initial data}

In order to start the evolution one has to determine the initial data. So far, the numerical methods used for their construction are based entirely on Theorem 7. It is assumed that the extrinsic curvature of the hyperboloidal initial surface is pure trace. Then the Yamabe equation is solved for a conformal factor, which chooses from a given conformal class of Riemannian metrics the one that has constant negative curvature. Finally, the other initial data are obtained from Equations (35, $36)$.

There are two problems with this kind of procedure. First, the Yamabe equation degenerates on the boundary. This is a difficult problem if one tries to prove existence of solutions of this equation, because the loss of ellipticity on the boundary means that one cannot simply appeal to known theorems. However, numerically this has not been a serious obstacle. The Yamabe equation is solved in a more or less standard way by using a Richardson iteration scheme to reduce the solution of the non-linear equation to a series of inversions of a linear operator.

The degeneracy of the equation on the boundary forces the solution and its derivative by regularity to have certain well defined boundary values. This and the global nature of the elliptic equations suggests that we use pseudo-spectral (or collocation) methods. Such methods are well suited for problems for which it is known beforehand that the solution will be sufficiently regular. Then, the solution can be expanded into a series of certain basis functions that are globally analytic. Therefore, the regularity conditions on the boundary are already built into the method. We refer readers interested in these methods to a recent review paper by Bonazzola et al. [25].

The more difficult problem in the determination of the initial data is the fact that the curvature components are obtained by successive division by $\Omega$. Since $\Omega$ vanishes on the boundary one has to compute a value which is of the form $0 / 0$. Although the theorem tells us that this is well defined 
(provided that some boundary conditions are satisfied), it still poses a numerical problem, because a straightforward implementation of l'Hôpital's rule loses accuracy due to the truncation error in the calculation of the numerator.

There exist two methods to overcome this problem. The first one $[47,51]$ is again based on pseudo-spectral methods. Essentially, in this approach the multiplication with the function $\Omega$ is expressed as a linear operation between the expansion coefficients of $\Omega$ and the other factor. The pseudo-inversion of this linear operator (in the sense of finding its Moore-Penrose inverse [123]) then corresponds to division by $\Omega$.

A different method for computing the quotient has been devised by Hübner [95]. Here, the problem of dividing by $\Omega$ has been reformulated into a problem for solving a second-order PDE for the quotient. This PDE is similar to the Yamabe equation in the sense that it also degenerates on the boundary where $\Omega$ vanishes, but it is linear.

Another consequence of the singularity of the Yamabe equation on $\mathscr{I}$ is the following. It is usually desirable to extend the solution of the Yamabe equation beyond $\mathscr{I}$ in such a way that it remains a solution of the equation. The reason is mainly that $\mathscr{I}$ is usually not aligned with the boundary of the computational domain so that it is impossible to restrict the calculation to the physical domain inside $\mathscr{I}$. Furthermore, the numerical stencils used in the discretisation of the evolution equations usually extend beyond $\mathscr{I}$ into the unphysical part of the computational domain, thus picking up information from the outside. Therefore, if the constraints are not satisfied outside $\mathscr{I}$ then it might be possible that constraint violating modes are excited. While the solution of the Yamabe equation inside $\mathscr{I}$ is uniquely determined, this is not so on the outside, where the solution is influenced by the choice of boundary conditions on the boundary of the computational grid. This means that the solution cannot be smooth across $\mathscr{I}$ unless the boundary conditions are chosen exactly right. But in general it is impossible to know the correct boundary values beforehand. It is guaranteed that the solution will be at least $\mathcal{C}^{2}$ across $\mathscr{I}$, but the third derivative may be discontinuous.

This situation does in fact occur as illustrated in Figure 12, which shows in the top picture a solution $u$ of the Yamabe equation in an axi-symmetric space-time. The $z$-axis $(r=0)$ is the axis of rotation, and the $r$-axis $(z=0)$ corresponds to the equatorial plane. The solution is assumed to be equatorially symmetric, so that it is obtained by specifying $u=1$ on the boundary $r=1$ and $z=1$ while using the symmetry conditions on the other parts of the computational grid. On the bottom are shown the third differences of $u$ in the $z$-direction. It is clearly visible that $\mathscr{I}$ is located at the quarter circle $r^{2}+z^{2}=0.8$. In Figure 13 we illustrate the "shielding" property of $\mathscr{I}$ for solutions of the Yamabe equation. Here is shown the difference of two solutions obtained with different boundary data on the outside. The solutions agree to a very high accuracy inside the physical domain. Some of these issues and possible ways to overcome them are discussed in further detail in [51].

\subsection{Evolution equations}

The initial data constructed in one of the ways described above must be evolved with the propagation equations extracted from the conformal field equations. As we remarked upon above, there are several ways to write the evolution equations, and therefore there are also several ways to implement them numerically. To date, there exist two different implementations. One is based on the ADM-like formulation of the conformal field equations, while the other uses a tetrad formalism. In the ADM-like formulation developed by Hübner [95] and Husa [100, 99], one introduces the coefficients of the intrinsic metric on the space-like slices and their extrinsic curvature as variables, while in the other formulation by Frauendiener [46], a tetrad and connection coefficients with respect to that tetrad are the basic variables. Concerning efficiency and usefulness, not much difference between these formulations is to be expected, provided the numerical schemes are the same. One

Living Reviews in Relativity

http: //www. livingreviews.org/Irr-2004-1/ 

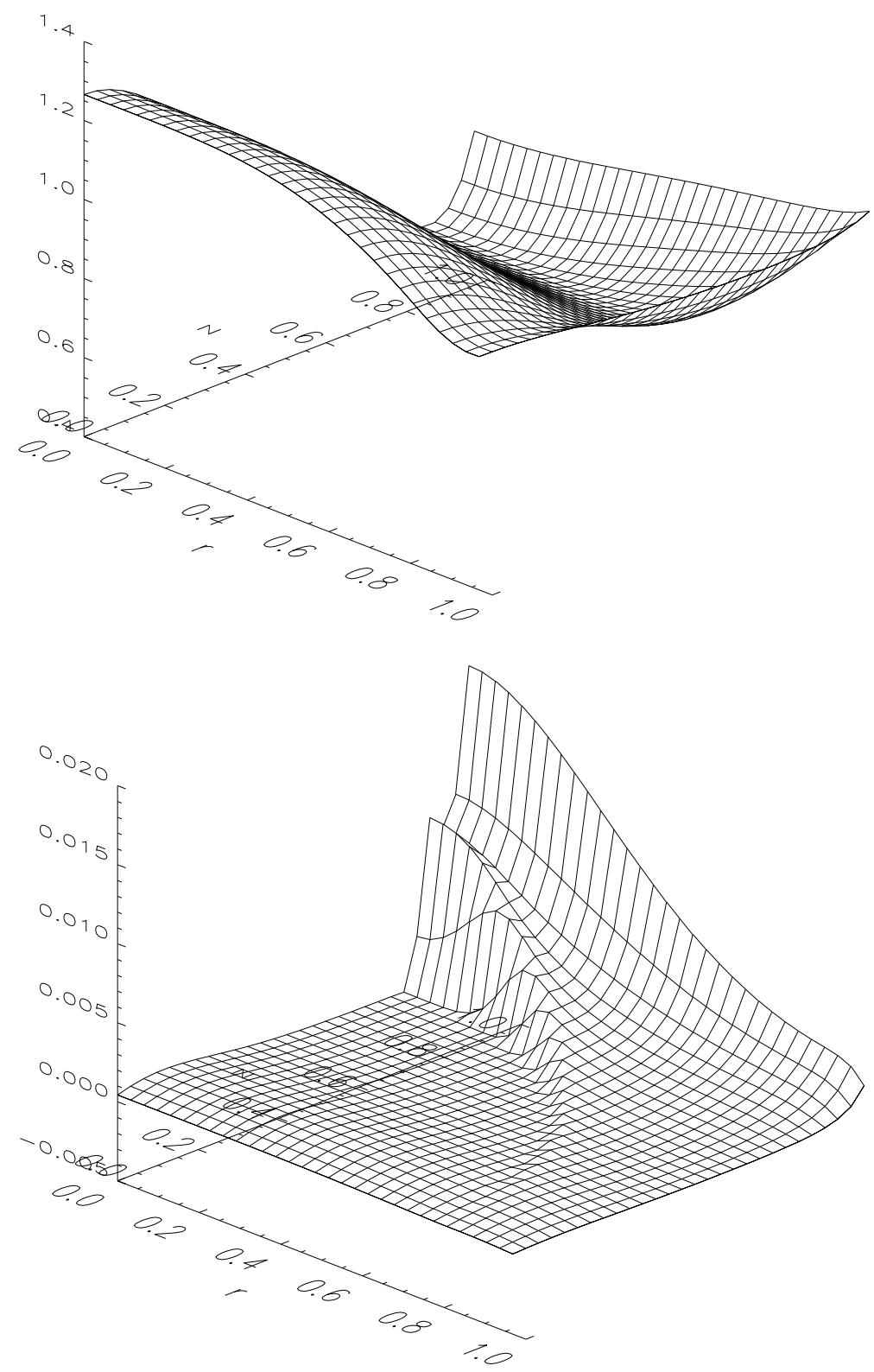

Figure 12: An axi-symmetric solution of the Yamabe equation with $u=1$ on the boundary (top) and its third differences in the $z$-direction (bottom). The location of $\mathscr{I}$ is clearly visible. 


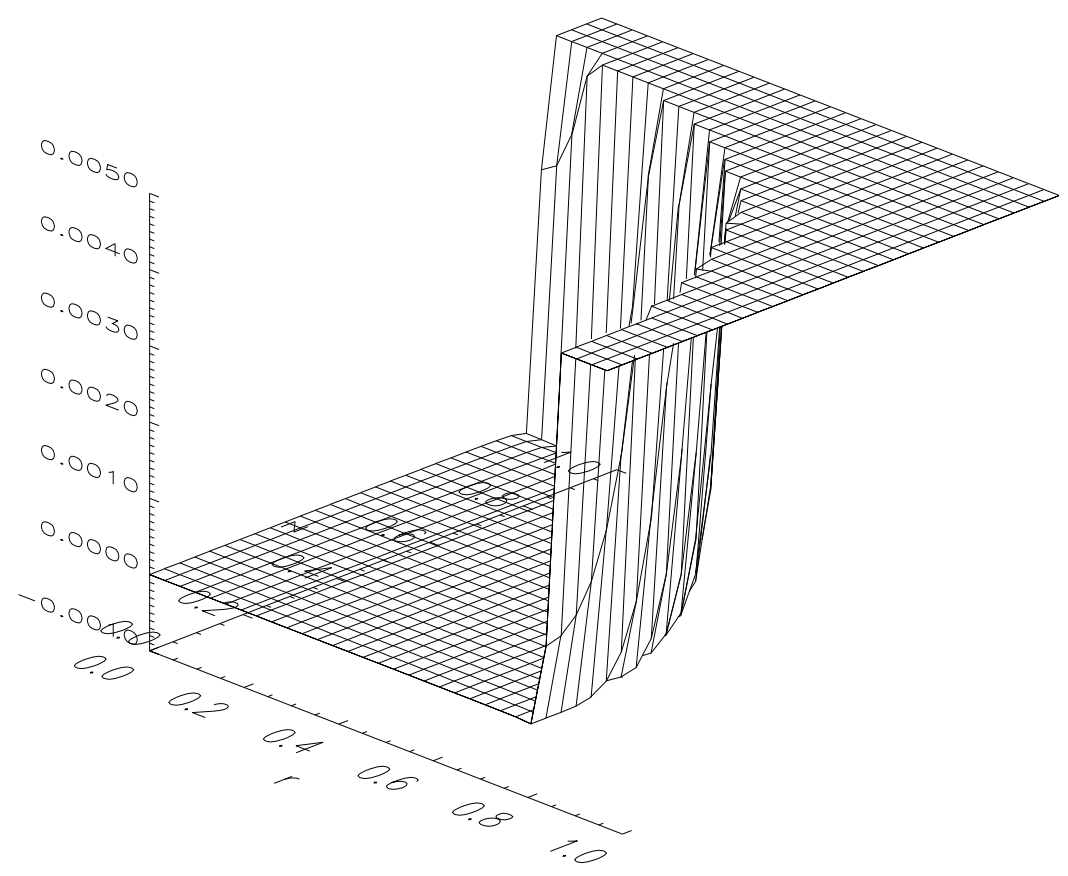

Figure 13: The difference between two solutions of the Yamabe equation obtained with different boundary conditions outside. Only the values less than 0.005 are shown.

minor advantage of the tetrad formulation might be the additional freedom in the orientation of the tetrad, which can be used on $\mathscr{I}$ to make the computational tetrad agree with the preferred tetrad on $\mathscr{I}$ (see below).

So far, the discretization of the equations has been rather straightforward. One of the schemes that has been used is the higher dimensional Lax-Wendroff scheme, also called the rotated Richtmyer scheme, a discretization scheme with second order accuracy. It has been employed alone [46] or together with Strang splitting [95] to treat the principal part of the equations differently from the source part. Since a second order scheme requires much more computing resources compared to higher order methods to achieve the same accuracy, Hübner started to use the method of lines [86] with a fourth order scheme to compute the spatial derivatives and fourth order Runge-Kutta for the evolution in time. He reports [95] that the fourth order method is very much superior to the second order scheme in terms of efficiency. (The feasibility of the method of lines in relativity has been studied by Hungerbühler [97], using pseudo-spectral methods for the spatial derivatives and a combination of Adams-Bashforth and Adams-Moulton schemes for the time evolution.) A similar method has been employed by Frauendiener and Hein [52] in a code that evolves the conformal field equations under the assumption of axi-symmetry. The code makes use of the "cartoon method" [2] (see also [50]), which can be used to treat axi-symmetric systems using Cartesian coordinates. The code has been used to reproduce exact solutions such as the boost-rotation symmetric solutions of Bičák and Schmidt [21, 22]. It allows one to compute the entire future of an initial hyperboloidal data set within that class up to time-like infinity $i^{+}$.

The conformal field equations and the propagation equations derived from them are quasi-linear. This implies that the characteristics of the system depend on the current solution, and this, in turn, means that one has to be able to change the time-step $\Delta t$ between successive time-slices in order to keep a stable evolution scheme. This is necessary because the schemes are explicit schemes and, therefore, subject to the Courant-Friedrichs-Lewy condition [37], which states that the numerical

Living Reviews in Relativity

http://www. livingreviews . org/Irr-2004-1/ 
domain of dependence of a point should always include the analytical domain of dependence. This requirement already excludes the popular leap-frog scheme, which is nevertheless used sometimes also for evolving the Einstein equations. A general criterion for computing the maximal time-step allowed in each iteration in arbitrary dimension has been derived in [46].

Another important point in the development of evolution codes is the numerical treatment of the boundaries. As explained already above, it is one of the advantages in the conformal approach that the outer boundary is not as influential as it is in the conventional approach using the standard Cauchy problem. It was also pointed out that in this case it is enough to impose a boundary condition that results in a numerically stable code because the outer boundary is located in the unphysical region and, therefore, cannot influence the physical space-time.

The proper way to treat the boundary is to prescribe conditions that are compatible with the full conformal field equations, in particular with their restriction to the boundary manifold. This has not been done so far. Since the outer boundary is not important for the physical effects, other ways of dealing with the boundary have been devised. One way is to forget about the restrictions of the conformal field equations to the boundary and to analyze the possible boundary conditions for the propagation equations. To first order, one can define ingoing and outgoing fields on the boundary. Then a sufficiently general boundary condition will be obtained by specifying the ingoing fields in terms of the outgoing ones. Although this boundary treatment is not necessarily compatible with the restriction of the conformal field equations to the boundary, it is compatible with the evolution equations. This means that the evolution can remain stable, although the solution will not satisfy the constraints in the domain of influence of the boundary, which, however, is always in the unphysical part of space-time. This method has been used in [46] with satisfactory results. In particular, the boundary did not give rise to non-physical modes. These findings are in agreement with the analysis of numerical boundary conditions by Trefethen [158].

Another method for dealing with the boundary has been found by Hübner [94]. He realized that it is sufficient to solve the conformal field equations in the physical space-time only, and not necessary to solve them in the unphysical region as long as the characteristics remain such that the information created in the unphysical part of the computational domain cannot reach the physical part. Consequently, in his treatment the grid is divided into three zones: the inner zone, the outer zone, and a transition zone. The inner zone covers the physical space-time (flagged by a positive conformal factor) and some part of the adjacent unphysical region. On this part of the grid the conformal field equations are solved. In the outer zone, which is located in a neighbourhood of the grid boundary, one solves an advection equation that propagates outwards, off the grid. In the transition zone, a sufficiently smooth interpolation between these two systems of equations is solved. The effect is that the boundary condition that has to be imposed on the grid boundaries is very simple, and that the noise generated in the transition region is propagated away from the physical region outward towards the grid boundary.

Our next point is concerned with the extraction of the radiative information from the numerically generated data. This is the part of the entire numerical process where the superiority of the conformal approach becomes apparent. How does one determine the radiative field? First of all, one needs to find $\mathscr{I}$ on the current time-slice. Since $\mathscr{I}$ is the surface on which the conformal factor $\Omega$ vanishes and since $\Omega$ is explicitly known during the evolution, the location of $\mathscr{I}$ is a simple task. The next problem is concerned with the orientation of the tetrad on $\mathscr{I}$. The asymptotic quantities are defined with respect to a specific geometrically characterized tetrad, a Bondi frame. But, in general, this tetrad is completely unrelated to the "computational" tetrad used for the evolution. Therefore, one needs to find the transformation from one to the other at each point of $\mathscr{I}$. Without going into too many details (see [46, 49, 48]), we remark that most asymptotic quantities, in particular the radiation field, are of a local character, so they can be read off without constructing a Bondi frame. This is rather fortunate because there are global issues involved in the transformation from the computational tetrad to the Bondi frame. These have implications 
for the determination of global quantities like the Bondi energy-momentum four-vector, but they have no effect on the radiation field, which is defined as that (complex) component of $K_{a b c d}$ that is entirely intrinsic to $\mathscr{I}$ :

$$
\psi_{4}=K_{a b c d} n^{a} \bar{m}^{b} n^{c} \bar{m}^{d} .
$$

Here, $n^{a}$ is a null-vector tangent to the generators of $\mathscr{I}$, i.e. $n_{a} \propto \nabla_{a} \Omega$, and $m^{a}$ is any complex space-like null-vector that is orthogonal to $n^{a}$. It is useful to require the space-like vector to be tangent to the intersection of $\mathscr{I}$ with the current time-slice. Augmenting these two vectors by a further real null-vector $l^{a}$ yields a null-tetrad which is fixed up to rotations in the (two-dimensional) tangent space of that intersection and boosts in the plane orthogonal to it. The behaviour of $\psi_{4}$ under these transformations is that of a GHP-weighted quantity [80, 134] with boost weight -2 and spin weight -2 . This corresponds to the quadrupole-like character of the gravitational radiation field. However, $\psi_{4}$ really depends only on the null-vector $n^{a}$. Suppose we perform a null-rotation around $n^{a}$, then $m^{a}$ transforms into $m^{a}+\alpha n^{a}$ for some complex valued function $\alpha$ on $\mathscr{I}$. But $\psi_{4}$ is invariant under this transformation. So in order to find $\psi_{4}$ it is only necessary to transform from the given computational tetrad to the tetrad specified above, which is rather straightforward. In fact, the computation of $\psi_{4}$ involves only the combination of certain components of the gravitational field with powers of $\nabla_{a} \Omega$.

The final step in the correct determination of the radiation is to find the correct time parameter. Suppose we follow a specific null generator of $\mathscr{I}$ crossing through successive time-slices. On each slice we compute $\psi_{4}$ on that generator. Then we obtain the radiation emitted by the source into the direction specified by the generator as a function of our computational time parameter. Since the time coordinate is rather arbitrary, this means that the waveform determined so far has no physical meaning. The problem is already present in Maxwell's theory: Suppose we have an emitter that sends out a pure sine wave. A detector far away from the source cannot determine the absolute frequency of the signal because the relative velocity of emitter and receiver might be non-zero, but the detector should also find a pure sine signal. However, this will be true only if the detector records the signal as a function of proper time. Any other time parameter along the detector's world-line will not produce a pure sine.

What one needs to do in the general case is to select among all parameters along the generator a specific, geometrically distinguished one, namely a Bondi parameter. A generator and such a parameter along it can be understood as a certain limit of freely falling observers with proper time clocks as they move towards infinity [49]. Bondi parameters are obtained as solutions of an ordinary, linear, second order differential equation, which is conformally invariant.

The computation of the Bondi energy-momentum is a global procedure, i.e. it depends on properties of the entire cut of $\mathscr{I}$ with the current time-slice. There are two steps involved in this procedure. First, one needs to obtain the asymptotic translation group (see e.g. [135]) on each cut. This provides four functions on the cut which are then, in a second step, integrated against the "mass aspect" which is another function obtained from the "Coulomb" part $\psi_{2}$ of the gravitational field, and the "news function," which is a combination of components of the Ricci tensor and connection coefficients. The first step, the determination of the translation group, is the global step because it involves solving a second order elliptic equation on the cut. These issues are discussed in more detail in [48].

All these procedures for finding the relevant data on $\mathscr{I}$ have been worked out analytically and they have also been tested (at least in part) numerically [46]. The tests have been performed under the assumption that null-infinity admits toroidal cuts, which has the advantage that one can actually compare the numerical results with analytical expressions because a whole class of exact solutions [152, 93] is known to exist. Admittedly, such space-times are rather unphysical, but since most of the extraction procedures are local there is no doubt that they will also work in more realistic cases.

Living Reviews in Relativity

http: //www . livingreviews . org/Irr-2004-1/ 


\subsection{Some numerical results}

Let us now discuss some of the achievements of the approach based on the conformal field equations in some more detail. They range from investigations of gravitational fields coupled to a scalar field in spherical symmetry to pure gravitational interactions studied in two space dimensions and, recently, in the most general case of three space dimensions. The numerical treatment of the conformal field equations was started by Hübner [89], who analyzed the asymptotic structure of spherically symmetric space-times in which a scalar field propagates under the influence of the gravitational attraction due to its own energy density. This system has been investigated rigorously in detail in earlier work by Christodoulou (see e.g. [30] and the references therein). Thus, the numerical results can be judged against the very detailed information found by analytical work. In all cases considered, the numerical results agree with the analytical ones. As a specific example, let us look at Figure 14 (taken from [92]) where the "upper part" of a space-time with a singularity is shown. It is obtained from the numerical evolution of initial data that are supercritical in the sense that the initial energy density (specified by a parameter $A$ ) is so large that the scalar field collapses down to a black hole.

This is indicated by the appearance of trapped surfaces and the subsequent formation of a singularity. The boundary of the region where the trapped surfaces exist is indicated by the thin line in the figure. It is the apparent horizon on which the divergence $\theta_{\text {out }}$ of the outgoing light rays vanishes. Note that this picture has been obtained by purely numerical methods. It should be compared with Figure 1 in Christodoulou's article [30].

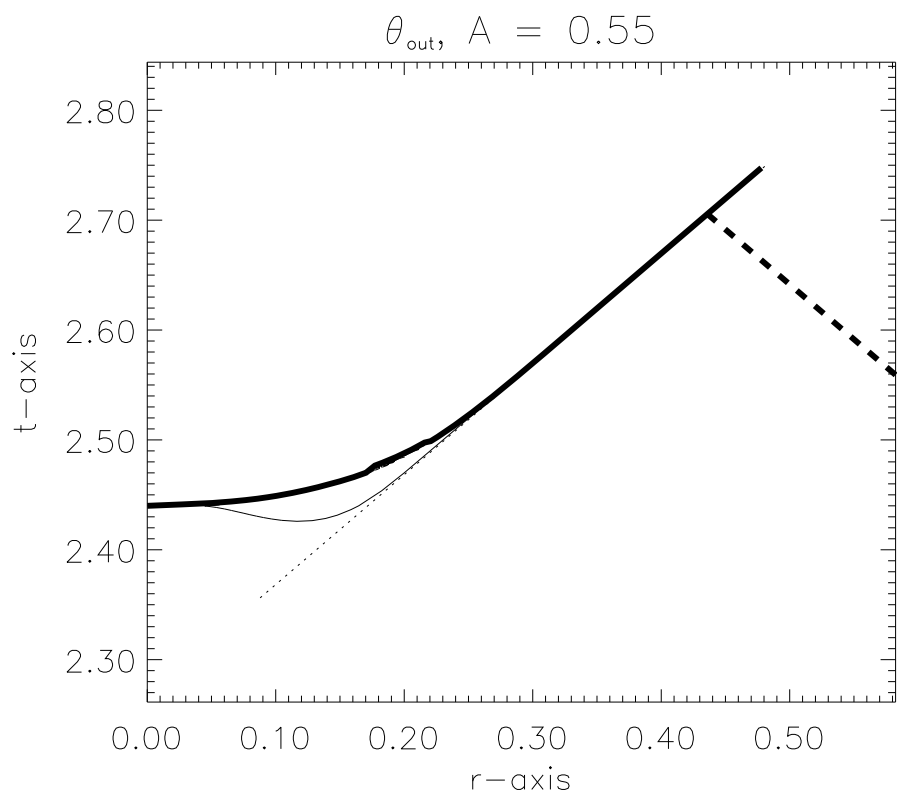

Figure 14: Upper corner of a space-time with singularity (thick line). The dashed line is $\mathscr{I}$, while the thin line is the locus of vanishing divergence of outgoing light rays, i.e. an apparent horizon.

Another part of the investigation was concerned with the radiation at infinity. In Figure 15 (also from [92]) the scalar radiation field at null-infinity as a function of proper time of an observer on $\mathscr{I}$ is shown.

In this example, the initial data are subcritical so that the scalar field, which initially collapses, subsequently disperses again. Note the long time-scale, ranging over approximately six orders of magnitude in proper time. This is a remarkable achievement because so far no other numerical 


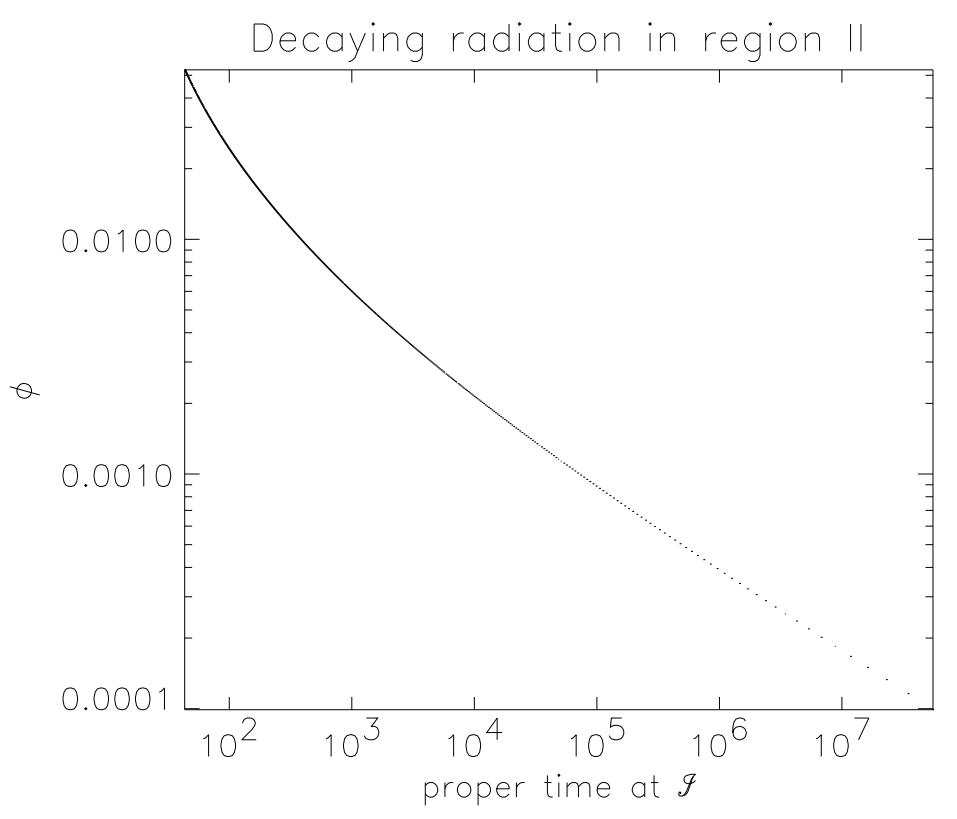

Figure 15: Decay of the radiation at null-infinity.

method has been able to monitor the evolution of relativistic space-times for such a long period of time.

The next step in the application of the conformal field equations to numerical problems was the implementation of $2 \mathrm{D}$ codes for the solution of A3-like space-times [45, 46]. These provide the first examples of vacuum space-times with gravitational radiation. Of course, they cannot be taken seriously as models of isolated systems because the topology of their $\mathscr{I}$ is not the physically distinguished $S^{2} \times \mathbb{R}$. However, they provided important test cases for the codes and in particular for methods to extract radiation. Since exact solutions with this kind of global structure are known [152, 93], one can again compare the numerical results with their exact counterparts. The radiation field $\psi_{4}$ and the Bondi mass for a particular case are shown in Figure 16.

In both diagrams the solid line is the exact solution while the dots indicate the computed values. Note that this was the first time that a fully non-linear waveform that agreed with an exact solution was computed. As a further example of the conformal method in numerical relativity, we consider the Schwarzschild space-time, which has recently been evolved with Hübner's 3D code [95]. Figure 17 is a numerical version of the Kruskal diagram, i.e. a diagram for the conformal structure of the Schwarzschild solution.

What is clearly visible here are the two null-infinities (blue lines) and the horizons (red lines). The green line is the "central" null-geodesic, i.e. the locus where the Kruskal null-coordinates $U$ and $V$ (see e.g. [163]) are equal. The dashed lines are "right going" null-geodesics, moving away from the left-hand $\mathscr{I}$. The diagram shows the cross-over where the two horizons (and the central line) intersect and, accordingly, we see a large part of the region III, which is below the cross-over, the regions I and IV with their corresponding $\mathscr{I}$ 's, and some part of region II where the future singularity is located. The non-symmetric look of the diagram is, of course, due to the fact that the coordinates used in the code have nothing to do with the Kruskal coordinates with respect to which one usually sees the Kruskal diagram of the extended Schwarzschild solution.

Husa [100] has used the code developed by Hübner to perform various parameter studies. Starting from weak perturbations of flat data that evolve into complete space-times with a regular

Living Reviews in Relativity

http: //www. livingreviews.org/lrr-2004-1/ 

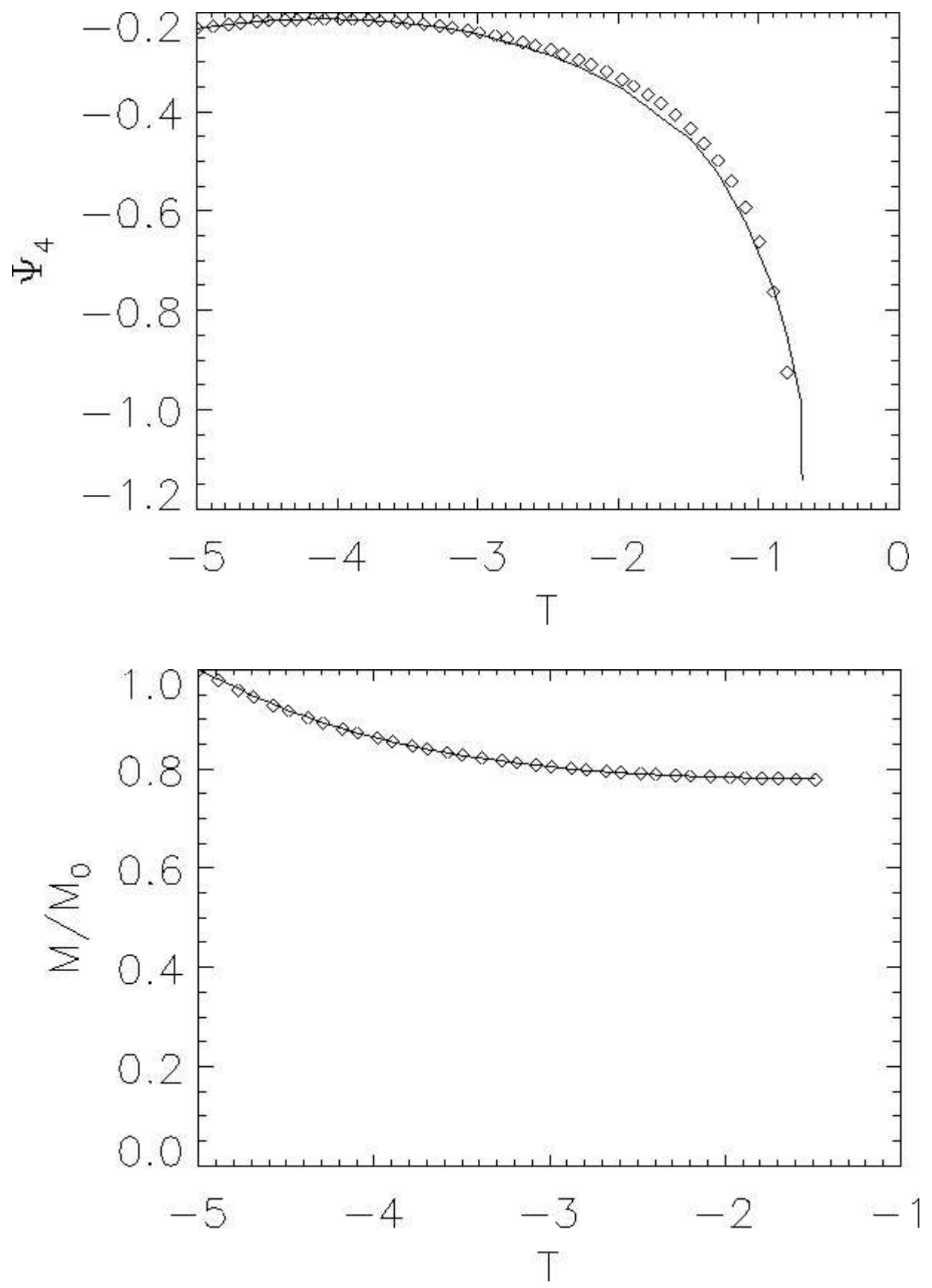

Figure 16: The radiation field $\psi_{4}$ and the Bondi mass for a radiating A3-like space-time. 




Figure 17: The numerically generated 'Kruskal diagram' for the Schwarzschild solution.

$i^{+}$, he studies the evolution of data obtained by increasing an "amplitude" and thereby increasing the deviation from flat data. He reports that stronger data rather quickly develop singularities which, however, are unphysical. This is suggested by the fact that the radiation decays quickly and that the news function still scales quadratically with the amplitude, which indicates that the data are in fact still weak. The origin of the singularities is due to an inappropriate choice of the gauge source functions, which - while adequate for the weak data - leads to a rapid growth of the lapse function outside of the physical space-time in the case of the stronger data. The cause of this growth is not known. It might be related to the fact that in the exterior region the constraints are not satisfied. In any case, this behaviour clearly indicates the importance of understanding the gauges that are used in the numerical implementations.

In [52] the question was considered as to what extent the boundaries in the unphysical region can influence the physical space-time. To this end, flat initial data are prescribed together with random boundary conditions on the grid boundary in the unphysical part. Then the square of the rescaled Weyl tensor is monitored. This should vanish everywhere inside the physical domain because the solution should be conformal to Minkowski space-time. The result of this calculation is shown in Figure 18.

This plot shows the square of the rescaled Weyl tensor depending on coordinate time $t$ and the distance $x$ from the symmetry axis in the equatorial plane $z=0$. Null-infinity is indicated by the black diagonal line running from $x \approx 1.3$ to $x=0$. The computation is carried out up to time-like infinity, where $\mathscr{I}$ meets the $t$-axis. The characteristic property of $\mathscr{I}$ is clearly visible.

In all the cases mentioned here, there is a clear indication that long-time studies of gravitational fields are feasible. All three cases have been checked against exact results (exact solutions or known theorems) so that there is no doubt that the numerical results are correct. These contributions show beyond any reasonable doubt that the conformal field equations can be used not only for the

Living Reviews in Relativity

http://www. livingreviews.org/lrr-2004-1/ 


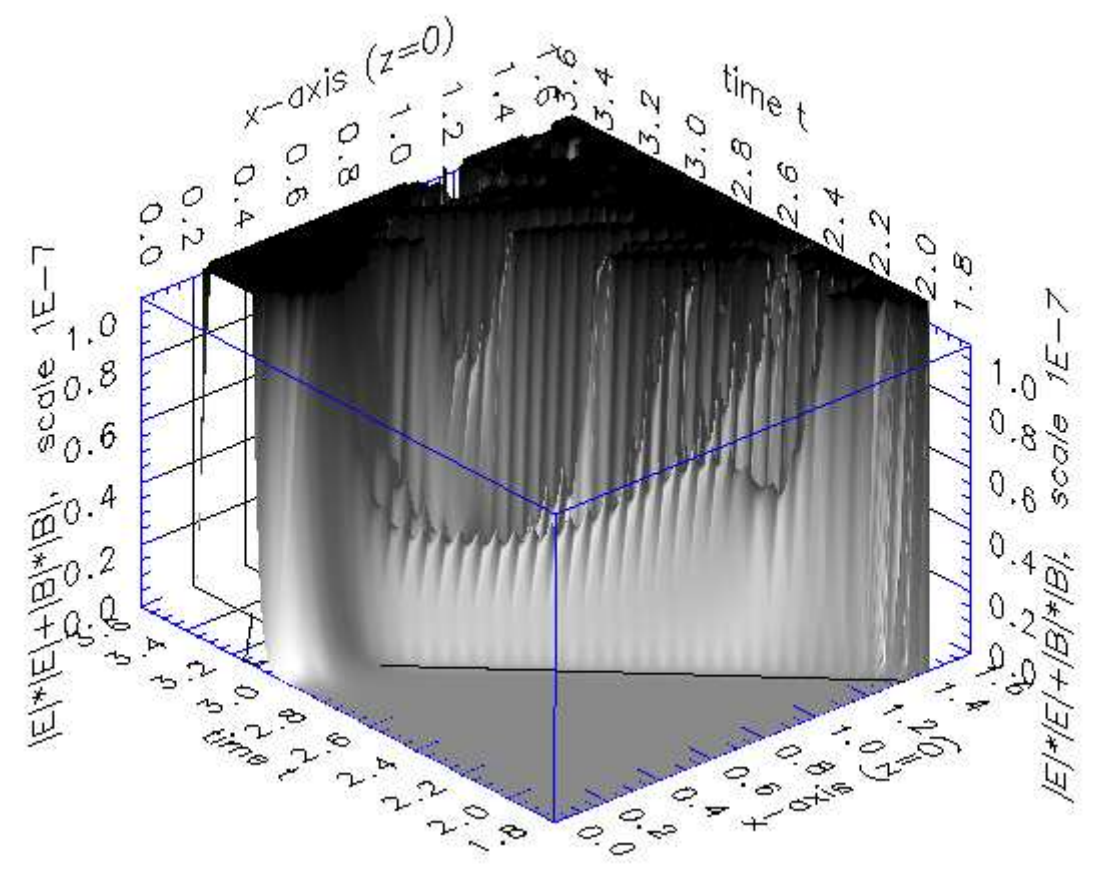

Figure 18: The rescaled Weyl tensor in a flat space-time obtained from flat initial data and random boundary data in the unphysical region.

analytical discussion of global properties of space-times, but also for the numerical determination of semi-global solutions. Clearly the problems with the artificial boundary have evaporated, the asymptotic region can accurately be determined, and the waveforms can be reliably computed. There is now good hope that, together with the analysis of $i^{0}$, the numerical computation of global space-times can be achieved in the near future.

\subsection{Problems for future considerations}

Now we want to discuss some of the problems that remain to be dealt with. Since the conformal approach has been tested numerically so far mostly in $2 \mathrm{D}$ cases with unphysical global structure, it is necessary to implement full 3D codes to run on general enough data. Work on this is well underway and first results that confirm the expectations based on the two-dimensional codes have been obtained [95, 100]. Husa and Lechner are currently developing a full 3D code using a method of lines approach to be integrated into the Cactus framework [111], which features an automated generator for the required right hand sides [98].

Apart from the requirement of having a general 3D code, there are other problems that require more consideration. Let us start with the boundary condition at the boundary of the computational domain. It would be interesting to see how the results of [72] translate to the conformal field equations. This would provide mathematically reasonable boundary conditions at the edge of the computational domain. Their implementation could result in stable codes that do not need any additional transition zones beyond $\mathscr{I}$ and that are compatible with the evolution equation, unlike the procedure used in [46].

Another problem has to do with the constraint equations. It is now a well-known fact in all numerical codes attempting to solve the Einstein equations that the conservation of the constraints, 
while analytically well established, is numerically not satisfied. This means that the constraints, even when they are prescribed exactly, will in the course of the evolution inadvertently drift away from the constraint surface, thereby no longer being preserved. And this means that the codes will not provide solutions of the full set of the field equations. This problem is present in the conformal approach as well. In a way, one could claim that this problem is not as serious as it might be in other approaches, for the simple reason that the computation is done in "conformal" time and not in physical time. Supposing we have chosen a conformal gauge that allows us to compute up to time-like infinity in a finite computational time, then we can (hopefully) control the deviation of the constraints.

Ultimately, this is, of course, not a solution and the source for the deviation of the constraints must be found. This means that one needs to study the system that propagates the constraints. The form of the general conformal field equations in the conformal Gauß gauge suggests that the crucial subset of equations is the Bianchi equation for the rescaled conformal tensor, because this is the only part that determines the wave properties of the gravitational field. Therefore, it should be interesting to study perturbations of the equations that propagate the constraints for this system in a reasonably simple case in order to see whether there are modes that diverge from the constraint hypersurface.

Another problem with the constraint equations is due to the particulars of the conformal approach. The necessity of dividing by the conformal factor to construct the initial data is annoying. The way around this problem is to solve the conformal constraints directly. While it has been possible to do this in the spherically symmetric case [90], no result is available for the general case. It would be very desirable to have another way of constructing the initial data, because then one could construct more easily data that evolve into space-times with multiple black holes. An interesting and certainly fruitful line of research would be to explore the new methods of treating the constraints, developed by Corvino and Schoen, and see whether they can be used for the construction of initial data. The fact that there are initial data that are exactly Schwarzschild outside a compact set, would allow us to dispense with the numerical division by the conformal factor in the computation of the data because these are known explicitly near $\mathscr{I}$.

As a last problem in connection with the conformal approach, one should mention that Friedrich's work on $i^{0}$ provides a way to evolve Cauchy data specified on an asymptotically Euclidean hypersurface to hyperboloidal initial data. A code that does that kind of evolution can provide the initial data for an evolution code for the hyperboloidal initial value problem. This area is entirely unexplored. Surely there will be difficult problems in the numerical treatment of the transport equations related to the total characteristic at spatial infinity. But the work on this problem is worthwhile because it would provide the final step to the ultimate goal of a global simulation of an isolated system.

A problem that affects all the work in numerical relativity today is the obscure nature of the gauge conditions. Currently, there is not much understanding of the effects of a gauge condition on the resulting nature of the coordinates (frame, conformal factor). Most of the work done on these problems is related to the choice of a lapse function; in particular several proposals have been made for selecting a time coordinate. These are mostly dictated by formal considerations such as the need to make a system hyperbolic or of easy implementation. To some extent this is justified because the physics cannot depend on the coordinates that are used. But it is also well known that there are "good" and "bad" coordinates. What "good" and "bad" means depends to a large extent on what the goal is.

Ideally, coordinates should be tied to the geometry so that they obtain a more invariant nature. In $1 \mathrm{D}$ cases one can set up a system of double null-coordinates (and a derived system of time and space coordinates). This provides a gauge that is good as long as the geometry is well behaved [90]. But, unfortunately, this gauge cannot be generalized in a straightforward way to higher dimensions (some attempts have been made in [46]). Probably one should assume a pragmatic viewpoint

Living Reviews in Relativity

http: //www . livingreviews . org/Irr-2004-1/ 
towards the problem of finding appropriate coordinates in the sense that one should regard the gauge sources as knobs that have to be adjusted by trial and error. Maybe it is possible, at least to some extent, to let the code do the "twiddling" automatically. This requires that one should be able to formulate criteria for a "good solution" that can be checked by the computer. Furthermore, it is also necessary that a change in the gauge sources does not change the characteristics of the system, because otherwise it is easy to get into situations where the system is not hyperbolic anymore.

Finally, there is no doubt that the issue of the correct boundary condition for codes based on the standard Einstein equations also needs more attention. Such codes should try to implement the boundary conditions given in [72]. Although it is not clear what these conditions mean physically, chances are good that they will produce stable codes (see e.g. the discussion of numerical boundary conditions in [86]). If this is the case, then one could try to check which ones work best by comparison with exact radiating solutions, such as the boost-rotation symmetric solutions discussed in [22]. Another test should be a numerical comparison with the data computed by a hyperboloidal evolution code. Such tests are important because so far the standard codes have been tested only against linearized solutions. This is the regime where one would expect them to work as the boundary condition is still benign. In this way, one could not only select physically reasonable boundary conditions for the standard codes, but also check how well they perform in comparison with the conformal codes. In particular, one could see how accurately the radiation extraction can be done with those codes and whether the accuracy is good enough for LIGO waveforms. Then one could compute them with a safe conscience using the standard codes, provided they are more efficient than the conformal codes. 


\section{Acknowledgments}

Most of this work was prepared while I was funded by a grant from DFG. I also benefitted from the MPI for Mathematics in the Sciences, Leipzig, where the article was completed. I wish to thank H. Friedrich for explaining some of the more subtle issues of null-infinity, and in particular of $i^{0}$, to me. Thanks are also due to P. Hübner for providing me with some of the figures in Section 4.4. I also want to thank Ted Newman and Roger Penrose for filling me in on some of the historical developments of the subject. Finally, I want thank M. Hein and T. Vogel for carefully reading through the manuscript and checking the equations.

This work was prepared partly during the workshop "Gravitational interaction of compact objects" at the KITP in Santa Barbara. It was supported in part by the National Science Foundation under Grant No. PHY99-07949.

Living Reviews in Relativity

http: //www. livingreviews.org/Irr-2004-1/ 


\section{Appendix: Reduction of the Conformal Field Equations}

In this appendix we show how to perform the reduction process for the conformal field equations to obtain the symmetric hyperbolic system of evolution equations and the constraints. We assume that we are given a time-like unit vector $t^{a}$ with respect to which the reduction is done. Any vector $v^{a}$ may be decomposed into parts perpendicular and parallel to $t^{a}$,

$$
v^{a}=\hat{v}^{a}+v t^{a}, \quad \text { with } \hat{v}^{a} t_{a}=0 \text { and } v=v^{a} t_{a},
$$

and similarly for one-forms $w_{a}$. We call a vector spatial with respect to $t^{a}$ if it is orthogonal to $t^{a}$. In particular, the metric $g_{a b}$ gives rise to a spatial metric $h_{a b}$ by the decomposition

$$
g_{a b}=h_{a b}+t_{a} t_{b}
$$

The volume four-form $\varepsilon_{a b c d}$ that is defined by the metric also gives rise to a decomposition as follows:

$$
\varepsilon_{a b c d}=4 \varepsilon_{[a b c} t_{d]} \Longleftrightarrow \varepsilon_{a b c}=\varepsilon_{a b c d} t^{d} .
$$

The covariant derivative operator $\nabla_{a}$ is written analogously,

$$
\nabla_{a}=D_{a}+t_{a} D
$$

thus defining two derivative operators: $D_{a}$ with $t^{a} D_{a}=0$ and $D=t^{a} \nabla_{a}$. The covariant derivative of $t^{a}$ itself is an important field. It gives rise to two component fields defined by

$$
\chi_{a}{ }^{b}=D_{a} t^{b}, \quad \chi^{b}=D t^{b} .
$$

Note that $\chi_{a}{ }^{b}$ is spatial in both its indices and that there is no symmetry implied between the two indices. Similarly, $\chi^{b}$ is automatically spatial.

It is useful to define two new derivative operators $\partial$ and $\partial_{a}$ by the following relations:

$$
\partial_{a} v_{b}=D_{a} v_{b}+t_{b} \chi_{a}{ }^{e} v_{e}-\chi_{a b} t^{e} v_{e}, \quad \partial v_{b}=D v_{b}+t_{b} \chi^{e} v_{e}-\chi_{b} t^{e} v_{e}
$$

These operators have the property that they are compatible with the spatial metric $h_{a b}$ and that they annihilate $t^{a}$ and $t_{a}$. If $t^{a}$ is the unit normal of a hypersurface, i.e. if $t_{a}$ is hypersurface orthogonal, then $\chi_{a b}$ is symmetric, $h_{a b}$ is the induced (negative definite) metric on the hypersurface, and $\partial_{a}$ is its Levi-Civita connection. In general this is not the case and so the operator $\partial_{a}$ possesses torsion. In particular, we obtain the following commutators (acting on scalars and spatial vectors):

$$
\begin{aligned}
2 \partial_{[a} \partial_{b]} f+2 \chi_{[a b]} \partial f & =0, \\
2 \partial_{[a} \partial_{b]} v_{c}+2 \chi_{[a b]} \partial v_{c}+h_{a}{ }^{r} h_{b}{ }^{s} h_{c}{ }^{t} R_{r s t}{ }^{d} v_{d}-2 \chi_{[a|c|} \chi_{b]}{ }^{e} v_{e} & =0 \\
{\left[\partial, \partial_{a}\right] f+\chi_{a} \partial f+\chi_{a}{ }^{c} \partial_{c} f } & =0 \\
{\left[\partial, \partial_{a}\right] v_{b}+\chi_{a} \partial v_{b}+\chi_{a}{ }^{c} \partial_{c} v_{b}-\chi_{b} \chi_{a}{ }^{e} v_{e}+\chi_{a b} \chi^{e} v_{e}+t^{c} h_{a}{ }^{e} h_{b}{ }^{f} R_{c e f}{ }^{d} v_{d} } & =0 .
\end{aligned}
$$

These commutators are obtained from the commutators between the derivative operators $D_{a}$ and $D$ by expressing them in terms of $\partial_{a}$ and $\partial$ on the one hand, and by the four-dimensional connection $\nabla_{a}$ on the other hand. This procedure yields two equations for the derivatives of $t^{a}$,

$$
\begin{aligned}
2 \partial_{[a} \chi_{b] c}+2 \chi_{[a b]} \chi_{c}+h_{a}{ }^{r} h_{b}{ }^{s} h_{c}{ }^{t} R_{r s t}{ }^{d} t_{d} & =0, \\
\partial \chi_{a b}-\partial_{a} \chi_{b}+\chi_{a}{ }^{c} \chi_{c b}+\chi_{a} \chi_{b}-t^{c} t^{d} h_{a}{ }^{r} h_{b}{ }^{s} R_{c r d s} & =0 .
\end{aligned}
$$

The information contained in the commutator relations and in the Equations (47) and (48) is completely equivalent to the Cartan equations for $\nabla_{a}$ that define the curvature and torsion tensors. 
This completes the preliminaries and we can now go on to perform the splitting of the equations. Our intention is to end up with a system of equations for all the spatial parts of the fields. In order not to introduce too many different kinds of indices, all indices refer to the four-dimensional space-time, but they are all spatial, i.e. any transvection with $t_{a}$ and $t^{a}$ vanishes. If we introduce hypersurfaces with normal vector $t^{a}$, then there exists an isomorphism between the tensor algebra on the hypersurfaces and the subalgebra of spatial four-dimensional tensors.

We start with the tensorial part of the equations. To this end we decompose the fields into various spatial parts and insert these decompositions into the conformal field equations defined by Equations (17, 18, 19, 20, 21). The fields are decomposed as follows:

$$
\begin{aligned}
K_{a b c d} & =4 t_{[a} E_{b][c} t_{d]}+2 t_{[a} B_{b]}{ }^{e} \varepsilon_{e c d}-2 \varepsilon_{a b}{ }^{e} B_{e[c} t_{d]}+\varepsilon_{a b e} E^{e f} \varepsilon_{f c d}, \\
\Phi_{a b} & =\phi_{a b}+2 t_{(a} \phi_{b)}+t_{a} t_{b} \phi \\
\Sigma_{a} & =\sigma_{a}+t_{a} \sigma .
\end{aligned}
$$

The function $\phi$ is fixed in terms of $\phi_{a b}$ because $\Phi_{a b}$ is trace-free.

Inserting the decomposition of $K_{a b c d}$ into Equation (18), decomposing the equations into various spatial parts, and expressing derivatives in terms of the operators $\partial_{a}$ and $\partial$ yields four equations:

$$
\begin{aligned}
\partial^{a} B_{a c}+\varepsilon_{e a d} E_{c}{ }^{e} \chi^{a d}+\varepsilon_{e c d} E_{a}{ }^{e} \chi^{a d} & =0, \\
\partial^{a} E_{a c}-\varepsilon_{e a d} B_{c}{ }^{e} \chi^{a d}-\varepsilon_{e c d} B_{a}{ }^{e} \chi^{a d} & =0, \\
\partial E_{b c}+\varepsilon_{a e(b} \partial^{a} B^{e}{ }_{c)}+2 \chi E_{b c}-2 \chi^{a}{ }_{(b} E_{c) a}+h_{b c} E_{a d} \chi^{a d}-E_{a(b} \chi_{c)}{ }^{a}+2 \varepsilon_{e a(b} B^{e}{ }_{c)} \chi^{a} & =0, \\
\partial B_{b c}-\varepsilon_{a e(b} \partial^{a} E^{e}{ }_{c)}+2 \chi B_{b c}-2 \chi^{a}{ }_{(b} B_{c) a}+h_{b c} B_{a d} \chi^{a d}-B_{a(b} \chi_{c)}{ }^{a}-2 \varepsilon_{e a(b} E^{e}{ }_{c)} \chi^{a} & =0 .
\end{aligned}
$$

Here we have defined $\chi=\chi_{a}{ }^{a}$. Treating the other fields and equations in a similar way, we obtain Equation (17) in the form of four equations:

$$
\begin{array}{r}
\partial \phi_{a}-\partial_{a} \phi-\chi^{e} \phi_{a e}+2 \chi_{a}{ }^{e} \phi_{e}+\chi_{a} \phi+\partial_{a} \Lambda+E_{a}{ }^{d} \sigma_{d}=0, \\
\partial_{[c} \phi_{a]}-\chi_{[c}{ }^{e} \phi_{a] e}+\chi_{[c a]} \phi-\frac{1}{2} \varepsilon_{c a e} B^{e d} \sigma_{d}=0, \\
\partial \phi_{a b}-\partial_{(a} \phi_{b)}+2 \chi_{(a} \phi_{b)}+\chi_{(a}{ }^{e} \phi_{b) e}-\chi_{(a b)} \phi-h_{a b} \partial \Lambda-E_{a b} \sigma-B_{(a}{ }^{e} \varepsilon_{b) d e} \sigma^{d}=0, \\
\partial_{[c} \phi_{a] b}-\chi_{[a c]} \phi_{b}+\phi_{[a} \chi_{c] b}-h_{b[a} \partial_{c]} \Lambda+\frac{1}{2} \varepsilon_{c a e} B_{b}^{e} \sigma-\frac{1}{2} \varepsilon_{c a e} E^{e f} \varepsilon_{f b d} \sigma^{d}=0 .
\end{array}
$$

The equation (19) for the conformal factor is rather straightforward. We obtain

$$
\begin{gathered}
\partial \Omega=\sigma, \\
\partial_{a} \Omega=\sigma_{a},
\end{gathered}
$$

while Equation (20) yields four equations:

$$
\begin{aligned}
\partial_{a} \sigma_{b}+\chi_{a b} \sigma-h_{a b} S+\Omega \phi_{a b} & =0 \\
\partial_{b} \sigma-\chi_{b}{ }^{e} \sigma_{e}+\Omega \phi_{b} & =0 \\
\partial \sigma_{b}+\chi_{b} \sigma+\Omega \phi_{b} & =0 \\
\partial \sigma-\chi^{e} \sigma_{e}-S+\Omega \phi & =0 .
\end{aligned}
$$

Finally, the equation (21) for $S$ gives two equations

$$
\begin{aligned}
\partial S+\phi_{b} \sigma^{b}+\phi \sigma-\Omega \partial \Lambda-2 \sigma \Lambda & =0, \\
\partial_{a} S+\phi_{a b} \sigma^{b}+\phi_{a} \sigma-\Omega \partial_{a} \Lambda-2 \sigma_{a} \Lambda & =0 .
\end{aligned}
$$

Living Reviews in Relativity

http: //www. livingreviews . org//rr-2004-1/ 
This completes the gauge-independent part of the equations. In order to deal with the gauges we now have to introduce an arbitrary tetrad and arbitrary coordinates. We extend the time-like unit vector to a complete tetrad $\left(t^{a}, e_{i}^{a}\right)$ with $t_{a} e_{i}^{a}=0$ for $i=1,2,3$. Let $x^{\mu}$ with $\mu=0,1,2,3$ be four arbitrary functions, which we use as coordinates. Application of $\partial$ and $\partial_{a}$ to the coordinates yields

$$
\partial x^{\mu}=c^{\mu}, \quad \partial_{a} x^{\mu}=c_{a}^{\mu} .
$$

The four functions $c^{\mu}$ and the four one-forms $c_{a}^{\mu}$ may be regarded as the 16 expansion coefficients of the tetrad vectors in terms of the coordinate basis $\partial_{\mu}=\partial / \partial x^{\mu}$ because of the identity

$$
\begin{aligned}
& \partial=c^{\mu} \partial_{\mu} \Longleftrightarrow \partial x^{\mu}=c^{\mu}, \\
& e_{i}^{a} \partial_{a}=c_{i}^{\mu} \partial_{\mu} \Longleftrightarrow e_{i}^{a} \partial_{a} x^{\mu}=e_{i}^{a} c_{a}^{\mu} .
\end{aligned}
$$

In a similar spirit, we apply the derivative operators to the tetrad and obtain

$$
\begin{aligned}
\partial e_{i}^{a} & =\Lambda^{a}{ }_{b} e_{i}^{b}, \\
\partial_{c} e_{i}^{a} & =\Gamma_{c}{ }^{a}{ }_{b} e_{i}^{b} .
\end{aligned}
$$

Again, transvection with $t^{a}$ on any index of $\Lambda^{a}{ }_{b}$ and $\Gamma_{c}{ }^{a}{ }_{b}$ vanishes. Furthermore, both $\Lambda_{a b}$ and $\Gamma_{c a b}$ are antisymmetric in their (last two) indices. Together with the 12 components of $\chi^{a}$ and $\chi_{a}{ }^{b}$ these fields provide an additional 12 components, which account for the 24 connection coefficients of the four-dimensional connection $\nabla_{a}$ with respect to the chosen tetrad.

Note that these fields are not tensor fields. They do not transform as tensors under the change of tetrad. Since we will keep the tetrad fixed here, we may, however, regard them as defining tensor fields whose components happen to coincide with them in the specified tetrad.

In order to extract the contents of the first of Cartan's structure equations, one needs to apply the commutators (43) and (45) to the coordinates to obtain

$$
\begin{aligned}
\partial c_{a}^{\mu}-\partial_{a} c^{\mu}+\chi_{a} c^{\mu}+\chi_{a}{ }^{b} c_{b}^{\mu} & =0 \\
\partial_{a} c_{b}^{\mu}-\partial_{b} c_{a}^{\mu}+2 \chi_{[a b]} c^{\mu} & =0 .
\end{aligned}
$$

Similarly, the second of Cartan's structure equations is exploited by applying the commutators to the tetrad vectors. Equation (16) is then used to substitute for the Riemann tensor in terms of the gravitational field, the trace-free part of the Ricci tensor, and the scalar curvature. Apart from the Equations (47) and (48), which come from acting on $t^{a}$, this procedure yields

$$
\begin{gathered}
\partial \Gamma_{a b c}-\partial_{a} \Lambda_{b c}-2 \Gamma_{a[b}{ }^{e} \Lambda_{c] e}+\chi_{a} \Lambda_{b c}+\chi_{a}{ }^{e} \Gamma_{e b c}+2 \chi_{a[b} \chi_{c]}+\Omega B_{a}{ }^{e} \varepsilon_{e b c}+2 h_{a[b} \phi_{c]}=0, \\
\partial_{a} \Gamma_{b c d}-\partial_{b} \Gamma_{a c d}+2 \chi_{[a b]} \Lambda_{c d}+2 \Gamma_{[a}{ }^{e}|c| \Gamma_{b] e d}+2 \chi_{[a \mid d} \chi_{b] c}+\Omega \varepsilon_{a b}{ }^{e} E_{e f} \varepsilon^{f} c d \\
-2 h_{c[a} \phi_{b] d}+2 h_{d[a} \phi_{b] c}+4 h_{c[a} h_{b] d} \Lambda=0 .
\end{gathered}
$$

Now we have collected all the equations that can be extracted from the conformal field equations and Cartan's structure equations. What remains to be done is to separate them into constraints and evolution equations. Before doing so, we notice that we do not have enough evolution equations for the tetrad components and the connection coefficients. The remedy to this situation is explained in Section 3.2. It amounts to adding appropriate "divergence equations". We obtain these by computing the "gauge source functions". The missing equations for the coordinates are obtained by applying the d'Alembert operator to the coordinates. Expressing the wave operator in terms of $\partial$ and $\partial_{a}$ yields the additional equations

$$
\partial c^{\mu}+\partial^{a} c_{a}^{\mu}=\chi^{a} c_{a}^{\mu}-\chi c^{\mu}+F^{\mu} .
$$


In order to find the missing equations for the tetrad we need to compute the gauge source functions

$$
\begin{aligned}
F_{0 k} & =g_{a b} \nabla^{c}\left(t^{a} \nabla_{c} e_{k}^{b}\right), \\
F_{i k} & =g_{a b} \nabla^{c}\left(e_{i}^{a} \nabla_{c} e_{k}^{b}\right) .
\end{aligned}
$$

In a similar way as explained above, we may regard these functions as components of tensor fields $F_{a b}$ and $F_{a}$ whose components happen to agree with them in the specified basis. Thus,

$$
F_{i k}=F_{a b} e_{i}^{a} e_{k}^{b}, \quad F_{0 i}=F_{a} e_{i}^{a} .
$$

Computing these tensor fields from $(78,79)$ gives

$$
\begin{aligned}
F_{a b} & =\partial \Lambda_{a b}+\partial^{e} \Gamma_{e a b}-\chi^{e} \Gamma_{e a b}+\chi \Lambda_{a b}, \\
F_{a} & =-\partial \chi_{a}-\partial^{e} \chi_{e a}+\chi^{e} \chi_{e a}-\chi_{a} \chi_{e}^{e}-\chi_{e c} \Gamma_{a}^{e c}-\chi^{c} \Lambda_{c a} .
\end{aligned}
$$

Now we are ready to collect the constraints:

$$
\begin{aligned}
\partial_{a} c_{b}^{\mu}-\partial_{b} c_{a}^{\mu}+2 \chi_{[a b]} c^{\mu} & =0, \\
\partial_{a} \Gamma_{b c d}-\partial_{b} \Gamma_{a c d}+2 \chi_{[a b]} \Lambda_{c d}-2 \Gamma_{[a|c|}{ }^{e} \Gamma_{b] e d}+2 \chi_{[a \mid d} \chi_{b] c}+\Omega \varepsilon_{a b}{ }^{e} E_{e f} \varepsilon^{f}{ }_{c d} & \\
-2 h_{c[a} \phi_{b] d}+2 h_{d[a} \phi_{b] c}+4 h_{c[a} h_{b] d} \Lambda & =0, \\
\partial_{a} \chi_{b c}-\partial_{b} \chi_{a c}+2 \chi_{[a b]} \chi_{c}-\Omega \varepsilon_{a b}{ }^{e} B_{e c}-2 h_{c[a} \phi_{b]} & =0, \\
\partial_{c} \phi_{a}-\partial_{a} \phi_{c}-2 \chi_{[c}{ }^{e} \phi_{a] e}+2 \chi_{[c a]} \phi-\varepsilon_{c a e} B^{e d} \sigma_{d} & =0, \\
\partial_{c} \phi_{a b}-\partial_{a} \phi_{c b}-2 \chi_{[a c]} \phi_{b}+2 \phi_{[a} \chi_{c] b}-2 h_{b[a} \partial_{c]} \Lambda+\varepsilon_{c a e} B^{e}{ }_{b} \sigma-\varepsilon_{c a e} E^{e f}{ }_{\varepsilon_{f b d}} \sigma^{d} & =0, \\
\partial^{a} B_{a c}+\varepsilon_{e a d} E_{c}{ }^{e} \chi^{a d}+\varepsilon_{e c d} E_{a}{ }^{e} \chi^{a d} & =0, \\
\partial^{a} E_{a c}-\varepsilon_{e a d} B_{c}{ }^{e} \chi^{a d}-\varepsilon_{e c d} B_{a}{ }^{e} \chi^{a d} & =0, \\
\partial_{a} \Omega-\sigma_{a} & =0, \\
\partial_{a} \sigma_{b}+\chi_{a b} \sigma-h_{a b} S+\Omega \phi_{a b} & =0, \\
\partial_{b} \sigma-\chi_{b}{ }^{e} \sigma_{e}+\Omega \phi_{b} & =0, \\
\partial_{a} S+\phi_{a b} \sigma^{b}+\phi_{a} \sigma-\Omega \partial_{a} \Lambda-2 \Lambda \sigma_{a} & =0 .
\end{aligned}
$$

Finally, we collect the evolution equations:

$$
\begin{aligned}
\partial c_{a}^{\mu}-\partial_{a} c^{\mu} & =-\chi_{a} c^{\mu}-\chi_{a}{ }^{b} c_{b}^{\mu}, \\
\partial c^{\mu}+\partial^{a} c_{a}^{\mu} & =\chi^{a} c_{a}^{\mu}-\chi c^{\mu}+F^{\mu} . \\
\partial \Gamma_{a b c}-\partial_{a} \Lambda_{b c} & =2 \Gamma_{a[b}{ }^{e} \Lambda_{c] e}-\chi_{a} \Lambda_{b c}-\chi_{a}{ }^{e} \Gamma_{e b c}-2 \chi_{a[b} \chi_{c]}-\Omega B_{a}{ }^{e} \varepsilon_{e b c}-2 h_{a[b} \phi_{c]}, \\
\partial \Lambda_{a b}+\partial^{e} \Gamma_{e a b} & =\chi^{e} \Gamma_{e a b}-\chi_{a b}+F_{a b}, \\
\partial \chi_{a}+\partial^{e} \chi_{e a} & =\chi^{e} \chi_{e a}-\chi_{a} \chi^{e}{ }_{e}-\chi_{e c} \Gamma^{e c}{ }_{a}-\chi^{c} \Lambda_{c a}-F_{a}, \\
\partial \chi_{a b}-\partial_{a} \chi_{b} & =-\chi_{a}{ }^{c} \chi_{c b}-\chi_{a} \chi_{b}-\Omega E_{a b}-\phi_{a b}-h_{a b}(\phi-2 \Lambda), \\
\partial \phi_{a}+\partial^{b} \phi_{a b} & =\chi^{e} \phi_{a e}-\chi_{c a} \phi^{c}-\phi_{a} \chi+\chi_{a} \phi_{c}{ }^{c}-3 \partial_{a} \Lambda, \\
\partial \phi_{a b}-\partial_{(a} \phi_{b)} & =-2 \chi_{(a} \phi_{b)}-\chi_{(a}{ }^{e} \phi_{b) e}+\chi_{(a b)} \phi+h_{a b} \partial \Lambda+E_{a b} \sigma+B_{(a}{ }^{e} \varepsilon_{b) d e} \sigma^{d}, \\
\partial E_{b c}+\varepsilon_{a e(b} \partial^{a} B^{e}{ }_{c)} & =-2 \chi E_{b c}+2 \chi^{a}{ }_{(b} E_{c) a}-h_{b c} E_{a d} \chi^{a d}+E_{a(b)} \chi_{c)}{ }^{a}-2 \varepsilon_{e a(b} B^{e}{ }_{c)} \chi^{a}, \\
\partial B_{b c}-\varepsilon_{a e(b} \partial^{a} E^{e}{ }_{c)} & =-2 \chi B_{b c}+2 \chi^{a}{ }_{(b} B_{c) a}-h_{b c} B_{a d} \chi^{a d}+B_{a(b} \chi_{c)}{ }^{a}+2 \varepsilon_{e a(b} E^{e}{ }_{c)} \chi^{a}, \\
\partial \Omega & =\sigma \\
\partial \sigma_{b} & =-\chi_{b} \sigma-\Omega \phi_{b}, \\
\partial \sigma & =\chi^{e} \sigma_{e}+S-\Omega \phi \\
\partial S & =-\phi_{b} \sigma^{b}-\phi \sigma+\Omega \partial \Lambda+2 \sigma \Lambda .
\end{aligned}
$$

Living Reviews in Relativity

http: //www. livingreviews . org/lrr-2004-1/ 
This is the complete system of evolution equations that can be extracted from the conformal field equations. As it is written, this system is symmetric hyperbolic. This is not entirely obvious but rather straightforward to verify. It is important to keep in mind that with our conventions the spatial metric $h_{a b}$ is negative definite. Altogether these are 65 equations. 


\section{Appendix: Conformal Rescalings and Curvature}

We compile here some formulae that are helpful for performing conformal rescalings. Suppose we rescale the given metric $\tilde{g}_{a b}$ to a new metric $g_{a b}=\theta^{2} \tilde{g}_{a b}$. Define $\Upsilon_{a}=\nabla_{a} \theta / \theta$. The Levi-Civita connection $\nabla_{a}$ of the new metric is given in terms of the Levi-Civita connection $\tilde{\nabla}_{a}$ of $\tilde{g}_{a b}$ by its action on an arbitrary vector field $v^{a}$,

$$
\nabla_{a} v^{b}=\tilde{\nabla}_{a} v^{b}+C_{a c}^{b} v^{c}=\tilde{\nabla}_{a} v^{b}+\left(\delta_{a}^{b} \Upsilon_{c}+\delta_{c}^{b} \Upsilon_{a}-g^{b d} g_{a c} \Upsilon_{d}\right) v^{c}
$$

From the action on vector fields we can obtain the action on tensors of arbitrary valence in the usual way.

Next, we consider the curvature tensor. It is useful to split the Riemann tensor into several pieces, which transform differently under conformal rescalings. We write

$$
R^{a b}{ }_{c d}=C_{c d}^{a b}-4 \delta_{[c}^{[a} P^{b]}{ }_{d]} .
$$

The tensor $C_{b c d}^{a}$ is, of course, Weyl's conformal tensor, characterised by the property of having the same symmetries as the Riemann tensor with all traces vanishing. The other piece, the tensor $P_{a b}$, can be uniquely expressed in terms of the Ricci tensor,

$$
P_{a b}=-\frac{1}{2}\left(R_{a b}-\frac{1}{6} g_{a b} R\right)=\Phi_{a b}-g_{a b} \Lambda
$$

The tensor $\Phi_{a b}$ is proportional to the trace-free part of the Ricci tensor, while $\Lambda=\frac{1}{24} R=-\frac{1}{4} g^{a b} P_{a b}$ is a multiple of the scalar curvature.

Under the conformal rescaling $\tilde{g}_{a b} \mapsto g_{a b}=\theta^{2} \tilde{g}_{a b}$, the different parts of the curvature transform as follows:

$$
\begin{aligned}
\tilde{C}_{b c d}^{a} & \mapsto C_{b c d}^{a}=\tilde{C}_{b c d}^{a}, \\
\tilde{P}_{a b} & \mapsto P_{a b}=\tilde{P}_{a b}-\tilde{\nabla}_{a} \Upsilon_{b}+\Upsilon_{a} \Upsilon_{b}-\frac{1}{2} \tilde{g}_{a b} \tilde{g}^{c d} \Upsilon_{c} \Upsilon_{d}
\end{aligned}
$$

Thus, the Weyl tensor is invariant under conformal rescalings. When $\tilde{P}_{a b}$ is expressed entirely in terms of the transformed quantities we get the relation

$$
\tilde{P}_{a b}=P_{a b}+\nabla_{a} \Upsilon_{b}+\Upsilon_{a} \Upsilon_{b}-\frac{1}{2} g_{a b} g^{c d} \Upsilon_{c} \Upsilon_{d}
$$

from which we can deduce (note that the contractions are performed with the transformed metric)

$$
\begin{gathered}
\tilde{\Phi}_{a b}=\Phi_{a b}+\frac{1}{\theta}\left(\nabla_{a} \nabla_{b} \theta-\frac{1}{4} g_{a b} \square \theta\right), \\
\tilde{\Lambda}=\theta^{2} \Lambda-\frac{1}{4} \theta \square \theta+\frac{1}{2} \nabla^{a} \theta \nabla_{a} \theta .
\end{gathered}
$$

Next consider the Bianchi identity $\nabla_{[e} R_{a b] c d}=0$. Inserting the decomposition (108) and taking appropriate traces allows us to write it as two equations,

$$
\begin{aligned}
\nabla_{a} C^{a}{ }_{b c d}+2 \nabla_{[c} P_{d] b} & =0, \\
\nabla_{a} P^{a}{ }_{b}-\nabla_{b} P^{a}{ }_{a} & =0 .
\end{aligned}
$$

Living Reviews in Relativity

http: //www. livingreviews . org/Irr-2004-1/ 


\section{References}

[1] Abrahams, A., Anderson, A., Choquet-Bruhat, Y., and York Jr, J.W., "Einstein and YangMills theories in hyperbolic form without gauge fixing", Phys. Rev. Lett., 75, 3377-3381, (1996). For a related online version see: A. Abrahams, et al., "Einstein and Yang-Mills theories in hyperbolic form without gauge fixing", (June, 1995), [Online Los Alamos Archive Preprint]: cited on December 19, 1999, http://arxiv.org/abs/gr-qc/9506072. 4.1

[2] Alcubierre, M., Brandt, S., Brügmann, B., Holz, D., Seidel, E., Takahashi, R., and Thornburg, J., "Symmetry without symmetry: Numerical simulation of axisymmetric systems using cartesian grids", Int. J. Mod. Phys. D, 10, 273-290, (2001). 4.3

[3] Andersson, L., and Chruściel, P.T., "On 'hyperboloidal' Cauchy data for vacuum Einstein equations and obstructions to smoothness of 'null-infinity"', Phys. Rev. Lett., 70(19), 28292832, (1993). For a related online version see: L. Andersson, et al., "On 'hyperboloidal' Cauchy data for vacuum Einstein equations and obstructions to smoothness of "null-infinity"', (April, 1993), [Online Los Alamos Archive Preprint]: cited on December 19, 1999, http: //arxiv.org/abs/gr-qc/9304019. 3.4

[4] Andersson, L., and Chruściel, P.T., "On hyperboloidal Cauchy data for vacuum Einstein equations and obstructions to smoothness of scri", Commun. Math. Phys., 161(3), 533-568, (1994). 3.4

[5] Andersson, L., Chruściel, P.T., and Friedrich, H., "On the regularity of solutions to the Yamabe equation and the existence of smooth hyperboloidal initial data for Einstein's field equations", Commun. Math. Phys., 149, 587-612, (1992). 3.4

[6] Arnowitt, R., Deser, S., and Misner, C.W., "The dynamics of general relativity", in Witten, Louis, ed., Gravitation: An Introduction to Current Research, 227-265, (Wiley, New York, NY, U.S.A., 1962). 4.1

[7] Ashtekar, A., "Asymptotic structure of the gravitational field at spatial infinity", in Held, A., ed., General Relativity and Gravitation, chapter 2, 37-70, (Plenum Press, New York, NY, U.S.A., 1980). $2.5,3.5$

[8] Ashtekar, A., "Asymptotic properties of isolated systems: recent developments", in Bertotti, B., de Felice, F., and Pascolini, A., eds., General Relativity and Gravitation, 37-68, (Reidel Publishing Company, Dordrecht, Netherlands, 1984). 2.5

[9] Ashtekar, A., Asymptotic quantization, (Bibliopolis, Naples, Italy, 1987). 2.5

[10] Ashtekar, A., Bombelli, L., and Reula, O., "The covariant phase space of asymptotically flat gravitational fields", in Francaviglia, M., ed., Mechanics, analysis and geometry: 200 years after Lagrange, 417-450, (North-Holland Publishing Co., Amsterdam, Netherlands, 1991). 2.5

[11] Ashtekar, A., and Hansen, R.O., "A unified treatment of null and spatial infinity. I. Universal structure, asymptotic symmetries and conserved quantities at spatial infinity", J. Math. Phys., 19, 1542-1566, (1978). 3.5

[12] Ashtekar, A., and Romano, J., "Spatial infinity as a boundary of space-time", Class. Quantum Grav., 9, 1069-1100, (1992). 3.5

[13] Ashtekar, A., and Streubel, M., "Symplectic geometry of radiative modes and conserved quantities at null infinity", Proc. R. Soc. London, Ser. A, 376, 585-607, (1981). 2.5 
[14] Ashtekar, A., and Xanthopoulos, B., "Isometries compatible with the asymptotic flatness at null infinity: A complete description", J. Math. Phys., 19, 2216-2222, (1978). 3.4

[15] Bartnik, R., "The spherically symmetric Einstein-Yang-Mills equations", in Perjes, Z., ed., Physics Today: Proceedings of the 1988 Hungarian Relativity Workshop, Tihany, 221-240, (Nova Science Publishers, New York, NY, U.S.A., 1992). 3.4

[16] Bateman, H., "The transformations of the electrodynamical equations", Proc. London Math. Soc. 2, 8, 223-264, (1910). 2.2

[17] Baumgarte, T.W., and Shapiro, S.L., "On the numerical integration of Einstein's field equations", Phys. Rev. D, 59, 024007, (1999). For a related online version see: T.W. Baumgarte, et al., "On the Numerical Integration of Einstein's Field Equations", (October, 1998), [Online Los Alamos Archive Preprint]: cited on December 19, 1999, http: //arxiv.org/abs/gr-qc/9810065. 4.1

[18] Beig, R., "Integration of Einstein's equations near spatial infinity", Proc. R. Soc. London, Ser. A, 391, 295-304, (1984). 3.5

[19] Beig, R., and Schmidt, B.G., "Einstein's equations near spatial infinity", Commun. Math. Phys., 87, 65-80, (1982). 3.5

[20] Beig, R., and Simon, W., "Proof of a multipole conjecture due to Geroch", Commun. Math. Phys., 78, 75-82, (1980). 3.5

[21] Bičák, J., Hoenselaers, C., and Schmidt, B.G., "The solutions of the Einstein equations for uniformly accelerated particles without nodal symmetries. II. Self-accelerating particles", Proc. R. Soc. London, Ser. A, 390, 411-419, (1983). 4.3

[22] Bičák, J., and Schmidt, B.G., "Asymptotically flat radiative space-times with boost-rotation symmetry", Phys. Rev. D, 40, 1827-1853, (1989). 3.4, 4.3, 4.5

[23] Bishop, N.T., "Some aspects of the characteristic initial value problem", in d' Inverno, R.A., ed., Approaches to Numerical Relativity, 20-33, (Cambridge University Press, Cambridge, U.K., 1993). 4.1

[24] Bishop, N.T., Gómez, R., Isaacson, R.A., Lehner, L., Szilagy, B., and Winicour, J., "Cauchy Characteristic Matching", in Iyer, B., ed., On the black hole trail, 383-408, (Kluwer, Dodrecht, Netherlands, 1998). 4.1

[25] Bonazzola, S., Gourgoulhon, E., and Marck, J.-A., "Spectral methods in general relativistic astrophysics", J. Comput. Appl. Math., 109, 433-473, (1999). 4.2

[26] Bondi, H., Pirani, F.A.E., and Robinson, I., "Gravitational waves in general relativity III. Exact plane waves", Proc. R. Soc. London, Ser. A, 251, 519-533, (1959). 2.2

[27] Bondi, H., van der Burg, M.G.J., and Metzner, A.W.K., "Gravitational waves in general relativity VII. Waves from axi-symmetric isolated systems", Proc. R. Soc. London, Ser. A, 269, 21-52, (1962). 2.2

[28] Bonnor, W.B., and Rotenberg, M.A., "Gravitational waves from isolated sources", Proc. R. Soc. London, Ser. A, 289, 247-274, (1966). 2.2

[29] Choquet-Bruhat, Y., and York, J.W., "The Cauchy Problem", in Held, A., ed., General Relativity and Gravitation, volume 1, chapter 4, 99-172, (Plenum Press, New York, NY, U.S.A., 1980). 3 
[30] Christodoulou, D., "The formation of black holes and singularities in spherically symmetric gravitational collapse", Commun. Pure Appl. Math., 44, 339-373, (1991). 4.4

[31] Christodoulou, D., and Klainermann, S., The global nonlinear stability of the Minkowski space, (Princeton University Press, Princeton, CA, U.S.A., 1993). 3.3

[32] Chruściel, P.T., and Delay, E., "Existence of non-trivial, vacuum, asymptotically simple space-times", Class. Quantum Grav., 19, L71-L79, (2002). For a related online version see: P.T. Chruściel, et al., "Existence of non-trivial, vacuum, asymptotically simple space-times", (March, 2002), [Online Los Alamos Archive Preprint]: cited on 18 March 2003, http:// arxiv.org/abs/gr-qc/0203053. 3.4

[33] Chruściel, P.T., and Delay, E., "Existence of non-trivial, vacuum, asymptotically simple spacetimes", Class. Quantum Grav., 19, 3389, (2002). 3.4

[34] Chruściel, P.T., MacCallum, M.A., and Singleton, D., "Gravitational waves in general relativity. XIV. Bondi expansions and the 'polyhomogeneity' of $\mathscr{I}$ ', Philos. Trans. R. Soc. London, Ser. A, 350(1692), 113-141, (1995). For a related online version see: P.T. Chruściel, et al., "Gravitational waves in general relativity. XIV. Bondi expansions and the "polyhomogeneity" of $\mathscr{I}$ ", (May, 1993), [Online Los Alamos Archive Preprint]: cited on December 19, 1999, http://arxiv.org/abs/gr-qc/9305021. 3.6

[35] Corvino, J., "Scalar curvature deformation and a gluing construction for the Einstein constraint equations", Commun. Math. Phys., 214, 137-189, (2000). 3.4, 3.5

[36] Corvino, J., and Schoen, R., "On the asymptotics for the Einstein constraint equations", (January, 2003), [Online Los Alamos Archive Preprint]: cited on 9 July 2003, http:// arxiv.org/abs/gr-qc/0301071. 3.4, 3.5

[37] Courant, R., Friedrichs, K.O., and Lewy, H., "Über die partiellen Differenzengleichungen der mathematischen Physik", Math. Ann., 100, 32-74, (1928). 4.3

[38] Cunningham, E., "The principle of relativity in electrodynamics and an extension thereof", Proc. London Math. Soc. 2, 8, 77-98, (1910). 2.2

[39] Cutler, C., and Wald, R.M., "Existence of radiating Einstein-Maxwell solutions which are $\mathcal{C}^{\infty}$ on all of $\mathscr{I}^{-}$and $\mathscr{I}^{+} "$, Class. Quantum Grav., 6, 453-466, (1989). 3.4

[40] Dixon, W. G., "Analysis of the Newman-Unti integration procedure for asymptotically flat space-times", J. Math. Phys., 11, 1238-1248, (1970). 2.2

[41] Ehlers, J., and Sachs, R.K., "Erhaltungssätze für die Wirkung in elektromagnetischen und gravischen Strahlungsfeldern", Z. Phys., 155, 498-506, (1959). 2.2

[42] Einstein, A., "Über Gravitationswellen", Sitz. Ber. Preuss. Akad. Wiss., 154-167, (1918). 2.2

[43] Engquist, B., and Majda, A., "Absorbing boundary conditions for the numerical simulation of waves", Math. Comput., 31(139), 629-651, (1977). 4.1

[44] Frauendiener, J., "Geometric description of energy-momentum pseudotensors", Class. Quantum Grav., 6, L237-L241, (1989). 2.2 
[45] Frauendiener, J., "Numerical treatment of the hyperboloidal initial value problem for the vacuum Einstein equations. I. The conformal field equations", Phys. Rev. D, 58, 064002, (1998). For a related online version see: J. Frauendiener, "Numerical treatment of the hyperboloidal initial value problem for the vacuum Einstein equations. I. The conformal field equations", (December, 1997), [Online Los Alamos Archive Preprint]: cited on December 19, 1999, http://arxiv.org/abs/gr-qc/9712050. 3.2, 4, 4.4

[46] Frauendiener, J., "Numerical treatment of the hyperboloidal initial value problem for the vacuum Einstein equations. II. The evolution equations", Phys. Rev. D, 58, 064003, (1998). For a related online version see: J. Frauendiener, "Numerical treatment of the hyperboloidal initial value problem for the vacuum Einstein equations. II. The evolution equations", (December, 1997), [Online Los Alamos Archive Preprint]: cited on December 19, 1999, http://arxiv.org/abs/gr-qc/9712052. 3.2, 4, 4.1, 4.3, 4.4, 4.5

[47] Frauendiener, J., "Calculating initial data for the conformal field equations by pseudospectral methods", J. Comput. Appl. Math., 109(1-2), 475-491, (1999). For a related online version see: J. Frauendiener, "Calculating initial data for the conformal field equations by pseudo-spectral methods", (June, 1998), [Online Los Alamos Archive Preprint]: cited on December 19, 1999, http://arxiv.org/abs/gr-qc/9806103. 4, 4.2

[48] Frauendiener, J., Conformal methods in numerical relativity, Habilitationsschrift, (Universität Tübingen, Tübingen, Germany, 1999). 4, 4.3

[49] Frauendiener, J., "Numerical treatment of the hyperboloidal initial value problem for the vacuum Einstein equations. III. On the determination of radiation", Class. Quantum Grav., 17(2), 373-387, (2000). For a related online version see: J. Frauendiener, "Numerical treatment of the hyperboloidal initial value problem for the vacuum Einstein equations. III. On the determination of radiation", (August, 1998), [Online Los Alamos Archive Preprint]: cited on December 19, 1999, http://arxiv.org/abs/gr-qc/9808072. 4, 4.1, 4.3

[50] Frauendiener, J., "On discretizations of axisymmetric systems", Phys. Rev. D, 66, 104027, (2002). For a related online version see: J. Frauendiener, "On discretizations of axisymmetric systems", (June, 2003), [Online Los Alamos Archive Preprint]: cited on 22 July 2003, http: //arxiv.org/abs/gr-qc/0207092. 4.3

[51] Frauendiener, J., "Some aspects of the numerical treatment of the conformal field equations", in Frauendiener, J., and Friedrich, H., eds., The conformal structure of space-times: Geometry, Analysis, Numerics, volume 604 of Lecture Notes in Physics, 261-282, (Springer-Verlag, Heidelberg, Germany, 2002). 4.2, 4.2

[52] Frauendiener, J., and Hein, M., "Numerical simulation of axisymmetric isolated systems in General Relativity", Phys. Rev. D, 66, 124004, (2002). For a related online version see: J. Frauendiener, et al., "Numerical simulation of axisymmetric isolated systems in General Relativity", (June, 2002), [Online Los Alamos Archive Preprint]: cited on 22 July 2003, http://arxiv.org/abs/gr-qc/0207094. 4.3, 4.4

[53] Friedrich, H., "Radiative gravitational fields and asymptotically static or stationary initial data", (April, 2003), [Online Los Alamos Archive Preprint]: cited on 9 July 2003, http: //arxiv.org/abs/gr-qc/0304003. 3.6

[54] Friedrich, H., "On the regular and the asymptotic characteristic initial value problem for Einstein's vacuum field equations", in Walker, M., ed., Proceedings of the third Gregynog

Living Reviews in Relativity

http://www. livingreviews.org/Irr-2004-1/ 
relativity workshop, Gravitational Radiation Theory, number MPI-PAE / Astro 204 in MaxPlanck Green Report, (Max-Planck-Institut f. Physik und Astrophysik, München, Germany, 1979). 3.1

[55] Friedrich, H., "The asymptotic characteristic initial value problem for Einstein's vacuum field equations as an initial value problem for a first-order quasilinear symmetric hyperbolic system", Proc. R. Soc. London, Ser. A, 378, 401-421, (1981). 3.1, 1

[56] Friedrich, H., "On the regular and the asymptotic characteristic initial value problem for Einstein's vacuum field equations", Proc. R. Soc. London, Ser. A, 375, 169-184, (1981). $3.1,1$

[57] Friedrich, H., "On the existence of analytic null asymptotically flat solutions of Einstein's field equations", Proc. R. Soc. London, Ser. A, 381, 361-371, (1982). 3.3

[58] Friedrich, H., "Cauchy problems for the conformal vacuum field equations in general relativity", Commun. Math. Phys., 91, 445-472, (1983). 5

[59] Friedrich, H., "On the hyperbolicity of Einstein's and other gauge field equations", Commun. Math. Phys., 100, 525-543, (1985). 3.2, 2

[60] Friedrich, H., "On purely radiative space-times", Commun. Math. Phys., 103, 36-65, (1986). 3.3

[61] Friedrich, H., "On the existence of n-geodesically complete or future complete solutions of Einstein's field equations with smooth asymptotic structure", Commun. Math. Phys., 107, 587-609, (1986). 6

[62] Friedrich, H., "On static and radiative space-times", Commun. Math. Phys., 119, 51-73, (1988). $\quad 3.3$

[63] Friedrich, H., "On the global existence and the asymptotic behavior of solutions to the Einstein-Maxwell-Yang-Mills equations", J. Differ. Geom., 34, 275-345, (1991). 2, 2, 3.2, $3.6,4.1$

[64] Friedrich, H., "Asymptotic structure of space-time", in Janis, A.I., and Porter, J.R., eds., Recent Advances in General Relativity: Essays in Honour of E.T. Newman, 146-181, (Birkhäuser Inc., Boston, MA, U.S.A., 1992). 2.5

[65] Friedrich, H., "Einstein equations and conformal structure: Existence of anti-de Sitter-type space-times", J. Geom. Phys., 17, 125-184, (1995). 3.6

[66] Friedrich, H., "Hyperbolic reductions for Einstein's field equations", Class. Quantum Grav., 13, 1451-1469, (1996). 3.2, 3.2

[67] Friedrich, H., "Einstein's equation and conformal structure", in Huggett, S.A., Mason, L.J., Tod, K.P., Tsou, S.S., and Woodhouse, N.M.J., eds., The Geometric Universe: Science, Geometry and the Work of Roger Penrose, 81-98, (Oxford University Press, Oxford, U.K., 1998). $\quad 2.1,2.5$

[68] Friedrich, H., "Gravitational fields near space-like and null infinity", J. Geom. Phys., 24, 83-163, (1998). $\quad 3.5$ 
[69] Friedrich, H., "Conformal Einstein evolution", in Frauendiener, J., and Friedrich, H., eds., The conformal structure of space-times: Geometry, Analysis, Numerics, volume 604 of Lecture Notes in Physics, 1-50, (Springer-Verlag, Heidelberg, Germany, 2002). For a related online version see: H. Friedrich, "Conformal Einstein evolution", (September, 2002), [Online Los Alamos Archive Preprint]: cited on 9 July 2003, http://arxiv.org/abs/gr-qc/ 0209018. 3.6

[70] Friedrich, H., "Conformal geodesics on vacuum space-times", Commun. Math. Phys., 235, 513-543, (2003). For a related online version see: H. Friedrich, "Conformal geodesics on vacuum space-times", (January, 2002), [Online Los Alamos Archive Preprint]: cited on 9 July 2003, http://arxiv.org/abs/gr-qc/0201006. 3.5

[71] Friedrich, H., and Kánnár, J., "Bondi-type systems near space-like infinity and the calculation of the NP-constants", J. Math. Phys., 41(4), 2195-2232, (2000). For a related online version see: H. Friedrich, et al., "Bondi-type systems near space-like infinity and the calculation of the NP-constants", (November, 1999), [Online Los Alamos Archive Preprint]: cited on December 19, 1999, http://arxiv.org/abs/gr-qc/9910077. 2.5, 3.5

[72] Friedrich, H., and Nagy, G., "The initial boundary value problem for Einstein's vacuum field equations", Commun. Math. Phys., 201, 619-655, (1998). 4.1, 4.5

[73] Friedrich, H., and Schmidt, B.G., "Conformal geodesics in general relativity", Proc. R. Soc. London, Ser. A, 414(1846), 171-195, (1987). 3.5

[74] Frittelli, S., and Reula, O., "On the Newtonian limit of general relativity", Commun. Math. Phys., 166, 221-235, (1994). 4.1

[75] Geroch, R., "Local characterization of singularities in general relativity", J. Math. Phys., 9, 450-465, (1968). 2.3

[76] Geroch, R., "Multipole moments. I. Flat space", J. Math. Phys., 11(6), 1955-1961, (1970). 3.5

[77] Geroch, R., "Multipole moments. II. Curved space", J. Math. Phys., 11(8), 2580-2588, (1970). $\quad 3.5$

[78] Geroch, R., "Space-time structure from a global point of view", in Sachs, R.K., ed., General Relativity and Cosmology, 71-103, (Academic Press, New York, NY, U.S.A., 1971). 2.3

[79] Geroch, R., "Asymptotic Structure of Space-time", in Esposito, F.P., and Witten, L., eds., Asymptotic Structure of Space-Time, 1-105, (Plenum Press, New York, NY, U.S.A., 1977). $2,2.1,2.5,3.5$

[80] Geroch, R., Held, A., and Penrose, R., "A space-time calculus based on pairs of null directions", J. Math. Phys., 14, 874-881, (1973). 4.3

[81] Geroch, R., and Horowitz, G.T., "Asymptotically simple does not imply asymptotically minkowskian", Phys. Rev. Lett., 40(4), 203-206, (1978). 2.3

[82] Geroch, R., and Winicour, J., "Linkages in general relativity", J. Math. Phys., 22, 803-812, (1981). $\quad 2.5$

[83] Glass, E.N., and Goldberg, J.N., "Newman-Penrose constants and their invariant transformations", J. Math. Phys., 11(12), 3400-3412, (1970). 2.5

Living Reviews in Relativity

http://www. livingreviews . org//rr-2004-1/ 
[84] Goldberg, J.N., "Invariant transformations and Newman-Penrose constants", J. Math. Phys., 8(11), 2161-2166, (1967). 2.5

[85] Goldberg, J.N., "Invariant transformations, conservation laws and energy-momentum", in Held, A., ed., General Relativity and Gravitation, volume 1, chapter 15, 469-489, (Plenum Press, New York, NY, U.S.A., 1980). 2.5

[86] Gustafsson, B., Kreiss, H.-O., and Oliger, J., Time dependent problems and difference methods, (Wiley, New York, NY, U.S.A., 1995). 4.3, 4.5

[87] Hansen, R., "Multipole moments of stationary space-times", J. Math. Phys., 15, 46-52, (1974). $\quad 3.5$

[88] Hawking, S.W., and Ellis, G.F.R., The large scale structure of space-time, (Cambridge University Press, Cambridge, U.K., 1973). 2.3

[89] Hübner, P., Numerische und analytische Untersuchungen von (singulären,) asymptotisch flachen Raumzeiten mit konformen Techniken, $\mathrm{PhD}$ thesis, (Ludwig-MaximiliansUniversität, München, Germany, 1993). 4, 4.4

[90] Hübner, P., "Method for calculating the global structure of (singular) spacetimes", Phys. Rev. D, 53(2), 701-721, (1994). For a related online version see: P. Hübner, "Method for calculating the global structure of (singular) spacetimes", (September, 1994), [Online Los Alamos Archive Preprint]: cited on December 19, 1999, http://arxiv.org/abs/gr-qc/ 9409029. 2, 4, 4.1, 4.5

[91] Hübner, P., "General relativistic scalar-field models and asymptotic flatness", Class. Quantum Grav., 12(3), 791-808, (1995). For a related online version see: P. Hübner, "General relativistic scalar-field models and asymptotic flatness", (August, 1994), [Online Los Alamos Archive Preprint]: cited on December 19, 1999, http://arxiv.org/abs/gr-qc/9408012. $2,4,4.1$

[92] Hübner, P., "Numerical approach to the global structure of space-time", Helv. Phys. Acta, 69, 317-320, (1996). 4, 4.4, 4.4

[93] Hübner, P., "More about vacuum spacetimes with toroidal null infinities", Class. Quantum Grav., 15, L21-L25, (1998). 4.3, 4.4

[94] Hübner, P., "How to avoid artificial boundaries in the numerical calculation of black hole space-times", Class. Quantum Grav., 16(7), 2145-2164, (1999). For a related online version see: P. Hübner, "How to avoid artificial boundaries in the numerical calculation of black hole space-times", (April, 1999), [Online Los Alamos Archive Preprint]: cited on December 19, 1999, http://arxiv.org/abs/gr-qc/9804065. 3.2, 4, 4.3

[95] Hübner, P., "A scheme to numerically evolve data for the conformal Einstein equation", Class. Quantum Grav., 16(9), 2823-2843, (1999). For a related online version see: P. Hübner, "A scheme to numerically evolve data for the conformal Einstein equation", (March, 1999), [Online Los Alamos Archive Preprint]: cited on December 19, 1999, http://arxiv.org/ abs/gr-qc/9903088. $4,4.2,4.3,4.4,4.5$

[96] Huggett, S.A., Mason, L.J., Tod, K.P., Tsou, S.S., and Woodhouse, N.M.J., eds., The Geometric Universe: Science, Geometry and the Work of Roger Penrose, (Oxford University Press, Oxford, U.K., 1998). 2.5 
[97] Hungerbühler, R., Lösung kugelsymmetrischer Systeme in der Allgemeinen Relativitätstheorie mit Pseudospektralmethoden, Diplomarbeit, (Universität Tübingen, Tübingen, Germany, 1997). 4.3

[98] Husa, S., "Into thin air; climbing up a smooth route to null-infinity", (July, 2003), [Online HTML Page]: cited on 11 July 2003, http://online.kitp.ucsb.edu/online/gravity03/ husa/. Talk given at KITP Santa Barbara. 4.5

[99] Husa, S., "Numerical relativity with the conformal field equations", in Fernandez, L., and Gonzalez, L., eds., Proceedings of the Spanish Relativity meeting, Madrid, 2001, Lecture Notes in Physics, (Springer-Verlag, Heidelberg, Germany, 2002). For a related online version see: S. Husa, "Numerical relativity with the conformal field equations", (April, 2002), [Online Los Alamos Archive Preprint]: cited on 11 July 2003, http://arxiv.org/abs/gr-qc/ 0204057. To appear. 4.3

[100] Husa, S., "Problems and Successes in the Numerical Approach to the Conformal Field Equations", in Frauendiener, J., and Friedrich, H., eds., The conformal structure of space-times: Geometry, Analysis, Numerics, volume 604 of Lecture Notes in Physics, 239-259, (SpringerVerlag, Heidelberg, Germany, 2002). For a related online version see: S. Husa, "Problems and Successes in the Numerical Approach to the Conformal Field Equations", (April, 2002), [Online Los Alamos Archive Preprint]: cited on 11 July 2003, http://arxiv.org/abs/gr-qc/ 0204043. 4.3, 4.4, 4.5

[101] Isenberg, J., and Park, J., "Asymptotically hyberbolic nonconstant mean curvature solutions of the Einstein constraint equations", Class. Quantum Grav., 14, A189-A201, (1997). 3.4

[102] Jordan, P., Ehlers, J., and Sachs, R.K., "Beiträge zur Theorie der reinen Gravitationsstrahlung", Akad. Wiss. Lit. Mainz, Abh. Math. Nat. Kl., 1, 1-85, (1961). 2.2

[103] Kánnár, J., "Hyperboloidal initial data for the vacuum Einstein equations with cosmological constant", Class. Quantum Grav., 13(11), 3075-3084, (1996). 3.4

[104] Kánnár, J., "On the existence of $\mathcal{C}^{\infty}$ solutions to the asymptotic characteristic initial value problem in general elativity", Proc. R. Soc. London, Ser. A, 452, 945-952, (1996). 3

[105] Kozameh, C.N., "Dynamics of null surfaces in general relativity", in Dadhich, N., and Narlikar, J., eds., Gravitation and Relativity: At the turn of the Millennium. Proceedings of the GR-15 Conference, 139-152, (IUCAA, Pune, India, 1998). 2.5

[106] Lichnerowicz, A., Théories relativistes de la gravitation et de l'électromagnétisme, (Masson et Cie., Paris, France, 1955). 2.2

[107] Lichnerowicz, A., "Sur les ondes et radiations gravitationnelles", Comptes Rendus Acad. Sci., 246, 893-896, (1958). 2.2

[108] Marder, L., "Gravitational waves in general relativity I. Cylindrical waves", Proc. R. Soc. London, Ser. A, 244, 524-537, (1958). 2.2

[109] Marder, L., "Gravitational waves in general relativity II. The reflexion of cylindrical waves", Proc. R. Soc. London, Ser. A, 246, 133-143, (1958). 2.2

[110] Marder, L., "Gravitational waves in general relativity V. An exact spherical wave", Proc. R. Soc. London, Ser. A, 261, 91-96, (1961). 2.2

[111] Max Planck Institute for Gravitational Physics, "The Cactus Homepage", (2003), [Online HTML document]: cited on 11 July 2003, http://www.cactus.org/. 4.5

Living Reviews in Relativity

http://www. livingreviews . org/lrr-2004-1/ 
[112] McCarthy, P.J., "Representations of the Bondi-Metzner-Sachs group I. Determination of the representations", Proc. R. Soc. London, Ser. A, 330, 517-535, (1972). 2.2

[113] McCarthy, P.J., "Structure of the Bondi-Metzner-Sachs group", J. Math. Phys., 13(11), 1837-1842, (1972). 2.2

[114] McCarthy, P.J., "Representations of the Bondi-Metzner-Sachs group II. Properties and classification of the representations", Proc. R. Soc. London, Ser. A, 333, 317-336, (1973). 2.2

[115] McLennan, J.A., "Conformal invariance and conservation laws for relativistic wave equations for zero rest mass", Nuovo Cimento, 3, 1360-1379, (1956). 2.2

[116] Newman, E.T., "Heaven and its properties", Gen. Relativ. Gravit., 7, 107-111, (1976). 2.5

[117] Newman, E.T., and Penrose, R., "An approach to gravitational radiation by a method of spin coefficients", J. Math. Phys., 3, 896-902, (1962). Errata 4 (1963), 998. 2.2

[118] Newman, E.T., and Penrose, R., "Note on the Bondi-Metzner-Sachs group", J. Math. Phys., 7, 863-879, (1966). $\quad 2.2$

[119] Newman, E.T., and Penrose, R., "New conservation laws for zero rest-mass fields in asymptotically flat space-time", Proc. R. Soc. London, Ser. A, 305, 175-204, (1968). 2.2, 2.5

[120] Newman, E.T., and Tod, K.P., "Asymptotically flat space-times", in Held, A., ed., General Relativity and Gravitation, volume 2, chapter 1, 1-36, (Plenum Press, New York, NY, U.S.A., 1980). 2.5

[121] Newman, E.T., and Unti, T.W.J., "Behavior of asymptotically flat empty spaces", J. Math. Phys., 3, 891-901, (1962). 2.2

[122] Newman, R.P.A.C., "The global structure of simple space-times", Commun. Math. Phys., 123, $17-52,(1989) . \quad 2.3$

[123] Penrose, R., "A generalized inverse for matrices", Proc. Cambridge Philos. Soc., 51, 406-413, (1955). $\quad 4.2$

[124] Penrose, R., "A spinor approach to general relativity", Ann. Phys. (N. Y.), 10, 171-201, (1960). $\quad 2.2$

[125] Penrose, R., "The light cone at infinity", in Infeld, L., ed., Relativistic Theories of Gravitation, 369-373, (Pergamon Press, Oxford, U.K., 1964). 2.2

[126] Penrose, R., "Zero rest-mass fields including gravitation: asymptotic behaviour", Proc. R. Soc. London, Ser. A, 284, 159-203, (1965). 2.2, 2.3, 2.4

[127] Penrose, R., "Structure of space-time", in DeWitt, C.M., and Wheeler, J.A., eds., Battelle Rencontres, 121-235, (W.A. Benjamin, Inc., New York, NY, U.S.A., 1968). 2.3, 2.5

[128] Penrose, R., "Relativistic symmetry groups", in Barut, A.O., ed., Group Theory in non-linear Problems, chapter 1, 1-58, (Reidel Publishing Company, Dordrecht, Netherlands, 1974). 2.2, 2.5

[129] Penrose, R., "Nonlinear gravitons and curved twistor theory", Gen. Relativ. Gravit., 7, 31$52,(1976) . \quad 2.5$ 
[130] Penrose, R., "Null hypersurface initial data for classical fields of arbitrary spin and for general relativity", Gen. Relativ. Gravit., 12, 225-264, (1980). originally published in Aerospace Research Laboratories Report 63-56 (P. G. Bergmann). 2.2

[131] Penrose, R., "Quasi-local mass and angular momentum in general relativity", Proc. R. Soc. London, Ser. A, 381, 53-63, (1982). 2.5

[132] Penrose, R., "The central programme of twistor theory", Chaos Solitons Fractals, 10(2-3), 581-611, (1999). $\quad 2.5$

[133] Penrose, R., "Some remarks on twistor theory", in Harvey, A., ed., On Einstein's Path: Essays in Honor of Engelbert Schücking, chapter 25, 353-366, (Springer, New York, NY, U.S.A., 1999). 2.2

[134] Penrose, R., and Rindler, W., Spinors and Spacetime, volume 1, (Cambridge University Press, Cambridge, U.K., 1984). 3.5, 4.3

[135] Penrose, R., and Rindler, W., Spinors and Spacetime, volume 2, (Cambridge University Press, Cambridge, U.K., 1986). 2.2, 2.3, 2.5, 4.3

[136] Pirani, F.A.E., "Invariant formulation of gravitational radiation theory", Phys. Rev., 105, 1089-1099, (1957). 2.2

[137] Pirani, F.A.E., "Gravitational waves in general relativity IV. The gravitational field of a fast-moving particle", Proc. R. Soc. London, Ser. A, 252, 96-101, (1959). 2.2

[138] Rendall, A.D., "Local and global existence theorems for the Einstein equations", Living Rev. Relativity, 5, lrr-2002-6, (September, 2002), [Online Journal Article]: cited on 23 July 2003, http://www. livingreviews .org/lrr-2002-6. 3.6

[139] Robinson, D.C., "Conserved quantities of Newman and Penrose", J. Math. Phys., 10(9), 1745-1753, (1969). 2.5

[140] Rosen, N., "Plane polarised waves in the general theory of relativity", Phys. Z. Sowjetunion, 12, 366-372, (1937). 2.2

[141] Sachs, R.K., "Propagation laws for null and type III gravitational waves", Z. Phys., 157, 462-477, (1960). 2.2

[142] Sachs, R.K., "Gravitational waves in general relativity VI. The outgoing radiation condition", Proc. R. Soc. London, Ser. A, 264, 309-338, (1961). 2.2, 2.2

[143] Sachs, R.K., "Asymptotic symmetries in gravitational theories", Phys. Rev., 128, 2851-2864, (1962). 2.2

[144] Sachs, R.K., "Gravitational waves in general relativity VIII. Waves in asymptotically flat space-time", Proc. R. Soc. London, Ser. A, 270, 103-127, (1962). 2.2

[145] Sachs, R.K., "Characteristic initial value problem for gravitational theory", in Infeld, L., ed., Relativistic Theories of Gravitation, 93-105, (Pergamon Press, Oxford, U.K., 1964). 2.2, 3.3

[146] Sachs, R.K., "Gravitational radiation", in DeWitt, C.M., and DeWitt, B., eds., Relativity, Groups and Topology, 523-562, (Gordon and Breach, New York, NY, U.S.A., 1964). 2.2

[147] Sachs, R.K., and Bergmann, P.G., "Structure of particles in linearized gravitational theory", Phys. Rev., 112(2), 674-680, (1958). 2.2

Living Reviews in Relativity

http: //www. livingreviews . org/Irr-2004-1/ 
[148] Schmidt, B.G., "A new definition of conformal and projective infinity of space-times", Commun. Math. Phys., 36, 73-90, (1974). 2.3

[149] Schmidt, B.G., "Conformal bundle boundaries", in Esposito, F.P., and Witten, L., eds., Asymptotic structure of space-time, 429-440, (Plenum Press, New York, NY, U.S.A., 1977). 2.3

[150] Schmidt, B.G., "Asymptotic structure of isolated systems", in Ehlers, J., ed., Isolated Gravitating Systems in General Relativity, 11-49, (Academic Press, New York, NY, U.S.A., 1978). 2.5

[151] Schmidt, B.G., "On the uniqueness of boundaries at infinity of asymptotically flat spacetimes", Class. Quantum Grav., 8, 1491-1504, (1991). 2.3

[152] Schmidt, B.G., "Vacuum space-times with toroidal null infinities", Class. Quantum Grav., 13, 2811-2816, (1996). $\quad 4.3,4.4$

[153] Simon, W., and Beig, R., "The multipole structure of stationary space-times", J. Math. Phys., 24(5), 1163-1171, (1983). 3.5

[154] Sommers, P., "The geometry of the gravitational field at space-like infinity", J. Math. Phys., 19, 549-554, (1978). 3.5

[155] Streubel, M., "Conserved' quantities for isolated gravitational systems", Gen. Relativ. Gravit., 9(6), 551-561, (1978). 2.5

[156] Trautman, A., "Boundary Conditions at infinity for physical theories", Bull. Acad. Polon. Sci. Cl. III, 6, 403-406, (1958). 2.2

[157] Trautman, A., "Radiation and boundary conditions in the theory of gravitation", Bull. Acad. Polon. Sci. Cl. III, 6, 407-412, (1958). 2.2

[158] Trefethen, L.N., "Group velocity in finite difference schemes", SIAM Rev., 24, 113-136, (1982). $\quad 4.3$

[159] Trefethen, L.N., "Finite Difference and Spectral Methods for Ordinary and Partial Differential Equations", graduate textbook, privately published, (1996). 4.1

[160] Valiente Kroon, J.A., "A new class of obstructions to the smoothness of null infinity", (November, 2002), [Online Los Alamos Archive Preprint]: cited on 9 July 2003, http: //arxiv.org/abs/gr-qc/0211024. 3.5

[161] Valiente Kroon, J.A., "Conserved quantities for polyhomogeneous space-times", Class. Quantum Grav., 15, 2479-2491, (1998). For a related online version see: J.A. Valiente Kroon, "Conserved Quantities for Polyhomogeneous Space-Times", (May, 1998), [Online Los Alamos Archive Preprint]: cited on December 19, 1999, http://arxiv.org/abs/gr-qc/9805094. 3.6

[162] Valiente Kroon, J.A., "Logarithmic Newman-Penrose constants for arbitrary polyhomogeneous spacetimes", Class. Quantum Grav., 16, 1653-1665, (1999). For a related online version see: J.A. Valiente Kroon, "Logarithmic Newman-Penrose constants for arbitrary polyhomogeneous spacetimes", (December, 1998), [Online Los Alamos Archive Preprint]: cited on December 19, 1999, http://arxiv.org/abs/gr-qc/9812004. 3.6

[163] Wald, R.M., General Relativity, (Chicago University Press, Chicago, IL, U.S.A., 1984). 4.4 
[164] Winicour, J., "Characteristic evolution and matching", Living Rev. Relativity, 4, lrr-2001-3, (March, 2001), [Online Journal Article]: cited on 23 July 2003, http: //www . livingreviews . org/lrr-2001-3. 4.1, 4.1

[165] Winicour, J., "Angular momentum in general relativity", in Held, A., ed., General Relativity and Gravitation, volume 1, chapter 3, 71-96, (Plenum Press, New York, NY, U.S.A., 1980). 2.5

[166] Winicour, J., "Logarithmic asymptotic flatness", Found. Phys., 15, 605-616, (1985). 3.4 